\title{
Botryosphaeriaceae: Current status of genera and species
}

\author{
Dissanayake AJ ${ }^{1,3, *}$, Phillips AJL ${ }^{2, *, \#}$, Li XH ${ }^{1, \#}$, Hyde KD ${ }^{3}$ \\ ${ }^{1}$ Institute of Plant and Environment Protection, Beijing Academy of Agriculture and Forestry Sciences, Beijing 100097, \\ People's Republic of China. \\ ${ }^{2}$ University of Lisbon, Faculty of Sciences, Biosystems and Integrative Sciences Institute (BioISI), Campo Grande, \\ 1749-016 Lisbon, Portugal. \\ ${ }^{3}$ Center of Excellence in Fungal Research, Mae Fah Luang University, Chiang Rai 57100, Thailand. \\ * These authors contributed equally to this work. \\ ${ }^{\#}$ Corresponding authors: AJL Phillips, XH Li.
}

Dissanayake AJ, Phillips AJL, Li XH, Hyde KD 2016 - Botryosphaeriaceae: Current status of genera and species. Mycosphere 7(7), 1001-1073, Doi 10.5943/mycosphere/si/1b/13

\begin{abstract}
The Botryosphaeriaceae comprises endophytes, saprobes and plant pathogens. Recently, some taxa in Botryosphaeriaceae have undergone nomenclatural changes. This paper represents the first comprehensive overview of Botryosphaeriaceae since 2013. Twenty three genera and 187 species of Botryosphaeriaceae are listed alphabetically, brief notes for species in each genus are provided. Information is provided for the location of ex-type isolates, new host records and geographical distribution of species. Available DNA sequences from ex-type isolates are listed in a table and multilocus phylogenetic trees for major genera in Botryosphaeriaceae are provided.
\end{abstract}

Key words - Botryosphaeriales - phylogeny - systematics - taxonomy

\section{Introduction}

Theissen \& Sydow (1918) introduced the Botryosphaeriaceae for three genera, namely, Botryosphaeria, Phaeobotryon and Dibotryon. Over the years the family and genera have undergone several taxonomic revisions and updates. Introduction of new genera, together with the addition of separate names for sexual and asexual genera resulted in at least 78 genera within this family. In addition, the type genus (Botryosphaeria) was subject to several changes that broadened the concept resulting in a diverse range of morphologies, especially in the asexual states, associated with a single sexual genus. Indeed the concept of the sexual state incorporated taxa with ascospores that could be hyaline or coloured, septate or aseptae. As a result, the genus Botryosphaeria and the family Botryosphaeriaceae became unmanageable and confused.

In a phylogentic approach based on the 28s rRNA gene Crous et al. (2006) revealed 10 lineages within Botryophaeriaceae. They considered these lineages to represent individual genera that could be recognised on the morphology of their sexual morphs. The existing anamorph genera names were used for most of the genera but new names were introduced for Neofusicoccum and Pseudofusicoccum. However, some of the clades could not be fully resolved, notably the one comprising Diplodia/Lasiodiplodia/Tiarosporella. Nevertheless, that study provided a stable base for future work on systematics of the family. 
Phillips et al. (2008) resolved the status of species with brown ascospores, introduced or reinstated a further five genera in Botryosphaeriaceae and clarified the status of Diplodia and Lasiodiplodia. Damm et al. (2007) showed that Aplosporella resides in Botryosphaeriaceae, Rojas et al. (2008) determined that Endomelanconiopsis also resides there and Phillips \& Alves considered Melanops to be yet another genus. Liu et al. (2012) used a phylogenetic approach together with a study of herbarium specimens and introduced two further genera namely Botryobambusa and Cophinforma bringing the total number of genera known from culture to 21 .

Wikee et al. (2013) re-instated Phyllostictaceae for Phyllosticta, Slippers et al. (2013) introduced Saccharataceae for Saccharata, Aplosporellaceae for Aplosporella and Melanopsaceae for Melanops. Phillips et al. (2013) provided comprehensive descriptions, notes and phylogenies for the 17 genera and 110 species known from culture that remained in the family. Since then 6 new genera and 85 new species/species combinations have been introduced (Table 1). In this paper we provide an updated list of all genera and species known from culture in the Botryosphaeriaceae together with relevant data on types and ex-type cultures. We also include updated information on hosts and known geographic distribution. Backbone trees for the larger genera (Botryosphaeria, Diplodia, Dothiorella/Spencermartinsia, Lasiodiplodia and Neofusicoccum) are included as well as an overview tree of the Botryosphaeriales showing the currently accepted limits of the Botryosphaeriaceae. In preparing this paper we included novel taxa and taxonomic changes published up to and including 31 October 2016 as well as those published in this issue of Mycosphere.

\section{Materials and methods}

The genera and species included in Phillips et al. (2013) was used as the starting point. Genera and species not included in that paper, or introduced since 2013 were found in searches of MycoBank and the mycological literature in general. All names were checked for validity, and for the existence of cultures and relevant sequences in GenBank before they were included in the current list. All genera and species known from culture are listed in Table 1. The USDA fungal database (Farr \& Rossman 2016) was used to gather information on host association and geographic distribution. Additional new disease reports, distribution and hosts were retrieved from recent publications.

\section{Phylogenetic analysis}

Sequences of internal transcribed spacer (ITS), translation elongation factor (tefl- $\alpha$ ) and 28S large subunit ribosomal gene (LSU) were download from NCBI GenBank (https://www.ncbi.nlm.nih.gov/genbank/). An overview backbone phylogenetic tree for the order Botryosphaeriales was constructed from ITS, tefl- $\alpha$ and LSU. Separate phylogenetic trees of the larger genera (Botryosphaeria, Diplodia, Dothiorella/Spencermartinsia, Lasiodiplodia and Neofusicoccum) were constructed from combined ITS and tefl- $\alpha$ sequences.

Single gene regions were aligned with Clustal X1.81 (Thompson et al. 1997) and combined with BioEdit v. 7.0.9.0 (Hall 1999). Further alignment of the sequences was done using default settings of MAFFT v. 7 (Katoh \& Toh 2008; http://mafft.cbrc.jp/alignment/server/) and manually adjusted where necessary using BioEdit v. 7.0.9.0 (Hall 1999). Maximum Parsimony analysis (MP) was performed with PAUP (Phylogenetic Analysis Using Parsimony) v. $4.0 b 10$ (Swofford 2002). Gaps were treated as missing data and ambiguously aligned regions were excluded. Trees were inferred using the heuristic search option with Tree Bisection Reconnection branch swapping and 1000 random sequence additions. Maxtrees was set at 1000, branches of zero length were collapsed and all multiple parsimonious trees were saved. Descriptive tree statistics for parsimony (tree length, consistency index, retention index, rescaled consistency index, and homoplasy index) were calculated for trees generated under different optimality criteria.

The best model of evolution for each gene region was determined using MRMODELTEST v. 2.2 (Nylander 2004) and maximum likelihood analyses were performed in RAXML GUI v. 0.9b2 (Silvestro \& Michalak 2010). The RAXML analyses were run with a rapid bootstrap analysis 
Table 1 GenBank accession numbers of genera and species in Botryosphaeriaceae and representative genera and species in order Botryosphaeriales. Type species are given in bold typeface. Isolates and sequence accession numbers of Botryosphaeriaceae genera and species introduced since Phillips et al. (2013) are italicized.

\begin{tabular}{|c|c|c|c|c|}
\hline Species & Cultures & ITS & tefl- $\alpha$ & LSU \\
\hline \multicolumn{5}{|l|}{ Botryosphaeriaceae } \\
\hline Alanphillipsia aloeicola & CBS 138896 & КР004444 & $N / A$ & KP004472 \\
\hline Alanphillipsia aloeigena & CBS 136408 & KF777137 & $N / A$ & KF777193 \\
\hline Alanphillipsia aloes & CBS 136410 & KF777138 & N/A & KF777194 \\
\hline Alanphillipsia aloetica & CBS 136409 & KF777139 & $N / A$ & KF777195 \\
\hline Alanphillipsia euphorbiae & CBS 136411 & KF 777140 & $N / A$ & KF777196 \\
\hline Barriopsis archontophoenicis & MFLUCC 14-1164 & $K X 235306$ & KX235305 & $K X 235307$ \\
\hline Barriopsis fusca & CBS 174.26 & EU673330 & EU673296 & DQ377857 \\
\hline Barriopsis iraniana & IRAN 1448C & FJ919663 & FJ919652 & KF766318 \\
\hline Barriopsis iraniana & IRAN1449C & FJ919665 & FJ919654 & N/A \\
\hline Barriopsis tectonae & MFLUCC 12-0381 & KJ556515 & KJ556516 & $N / A$ \\
\hline Botryobambusa fusicoccum & MFLUCC 11-0143 & JX646792 & JX646857 & JX646809 \\
\hline Botryobambusa fusicoccum & MFLUCC 11-0657 & JX646793 & JX646858 & JX646810 \\
\hline Botryosphaeria agaves & MFLUCC 11-0125 & JX646791 & JX646856 & JX646808 \\
\hline Botryosphaeria agaves & MFLUCC 10-0051 & JX646790 & JX646855 & JX646807 \\
\hline Botryosphaeria auasmontanum & $C M W 25413$ & KF766167 & $E U 101348$ & KF766332 \\
\hline Botryosphaeria corticis & CBS 119047 & DQ299245 & EU017539 & EU673244 \\
\hline Botryosphaeria corticis & ATCC 22927 & DQ299247 & EU673291 & EU673245 \\
\hline Botryosphaeria dothidea & CBS 115476 & AY236949 & AY236898 & DQ377852 \\
\hline Botryosphaeria dothidea & CBS 110302 & AY259092 & AY573218 & EU673243 \\
\hline Botryosphaeria fabicerciana & CBS 127193 & HQ332197 & HQ332213 & N/A \\
\hline Botryosphaeria fabicerciana & CMW 27108 & HQ332200 & HQ332216 & N/A \\
\hline Botryosphaeria fusispora & MFLUCC 10-0098 & JX646789 & JX646854 & JX646806 \\
\hline Botryosphaeria fusispora & MFLUCC 11-0507 & JX646788 & JX646853 & JX646805 \\
\hline Botryosphaeria minutispermatia & GZCC 16-0013 & $K X 447675$ & $K X 447678$ & $N / A$ \\
\hline Botryosphaeria minutispermatia & GZCC 16-0014 & KX447676 & $K X 447679$ & $N / A$ \\
\hline Botryosphaeria ramosa & CBS 122069 & EU144055 & EU144070 & N/A \\
\hline Botryosphaeria scharifii & CBS 124703 & JQ772020 & JQ772057 & N/A \\
\hline Botryosphaeria scharifii & CBS 124702 & JQ772019 & JQ772056 & N/A \\
\hline Botryosphaeria sinensia & CGMCC 3.17722 & KT343254 & KU221233 & $N / A$ \\
\hline Botryosphaeria sinensia & CGMCC 3.17724 & KT343256 & $K U 221234$ & $N / A$ \\
\hline Cophinforma atrovirens & MFLUCC 11-0425 & JX646800 & JX646865 & JX646817 \\
\hline Cophinforma atrovirens & MFLUCC 11-0655 & JX646801 & JX646866 & JX646818 \\
\hline Cophinforma mamane & CBS 117444 & KF531822 & KF531801 & DQ377855 \\
\hline Diplodia africana & CBS 120835 & EF445343 & EF445382 & N/A \\
\hline Diplodia africana & CBS 121104 & EF445344 & EF445383 & N/A \\
\hline Diplodia agrifolia & CBS 132777 & JN693507 & JQ517317 & N/A \\
\hline Diplodia agrifolia & UCROK1429 & JQ411412 & JQ512121 & N/A \\
\hline Diplodia alatafructa & CBS 124931 & FJ888460 & FJ888444 & N/A \\
\hline Diplodia alatafructa & CBS 124933 & FJ888478 & FJ888446 & N/A \\
\hline Diplodia allocellula & CBS 130408 & JQ239397 & JQ239384 & JQ239410 \\
\hline Diplodia allocellula & CBS 130410 & JQ239399 & JQ239386 & JQ239412 \\
\hline Diplodia bulgarica & CBS 124254 & GQ923853 & GQ923821 & N/A \\
\hline Diplodia bulgarica & CBS 124135 & GQ923852 & GQ923820 & N/A \\
\hline Diplodia corticola & CBS 112549 & AY259100 & AY573227 & AY928051 \\
\hline Diplodia corticola & CBS 112546 & AY259110 & DQ458872 & EU673262 \\
\hline Diplodia crataegicola & MFLU 15-1311 & KT290244 & $K T 290248$ & $N / A$ \\
\hline Diplodia cupressi & CBS 168.87 & DQ458893 & DQ458878 & EU673263 \\
\hline
\end{tabular}




\begin{tabular}{|c|c|c|c|c|}
\hline Species & Cultures & ITS & tef1- $\alpha$ & LSU \\
\hline Diplodia cupressi & CBS 261.85 & DQ458894 & DQ458879 & EU673264 \\
\hline Diplodia estuarina & $C M W 41231$ & КP860831 & КР860676 & $N / A$ \\
\hline Diplodia estuarina & CMW41230 & KР860830 & KP860675 & $N / A$ \\
\hline Diplodia fraxinii & CBS 136010 & KF307700 & KF318747 & $N / A$ \\
\hline Diplodia galiicola & MFLU 15-1310 & $K T 290245$ & KT290249 & $N / A$ \\
\hline Diplodia insularis & CBS 140350 & KX833072 & $K X 833073$ & $N / A$ \\
\hline Diplodia intermedia & CBS 124462 & GQ923858 & GQ923826 & N/A \\
\hline Diplodia intermedia & CBS 124134 & HM036528 & GQ923851 & N/A \\
\hline Diplodia malorum & CBS 124130 & GQ923865 & GQ923833 & N/A \\
\hline Diplodia malorum & CBS 112554 & AY259095 & DQ458870 & N/A \\
\hline Diplodia mutila & CBS 112553 & AY259093 & AY573219 & AY928049 \\
\hline Diplodia mutila & CBS 230.30 & DQ458886 & DQ458869 & AY928049 \\
\hline Diplodia neojuniperi & CPC 22753 & KM006431 & KM006462 & $N / A$ \\
\hline Diplodia olivarum & CBS 121887 & EU392302 & EU392279 & N/A \\
\hline Diplodia olivarum & CBS 121886 & EU392297 & EU392274 & N/A \\
\hline Diplodia pseudoseriata & CBS 124906 & EU080927 & EU863181 & N/A \\
\hline Diplodia pseudoseriata & CBS 124907 & EU080922 & EU863179 & N/A \\
\hline Diplodia quercivora & CBS 133852 & JX894205 & JX894229 & N/A \\
\hline Diplodia rosacearum & CBS 141915 & KT956270 & KU378605 & $N / A$ \\
\hline Diplodia rosulata & CBS 116470 & EU430265 & EU430267 & DQ377896 \\
\hline Diplodia rosulata & CBS 116472 & EU430266 & EU430268 & DQ377897 \\
\hline Diplodia sapinea & CBS 393.84 & DQ458895 & DQ458880 & DQ377893 \\
\hline Diplodia sapinea & CBS 109725 & DQ458896 & DQ458881 & EU673270 \\
\hline Diplodia scrobiculata & CBS 118110 & AY253292 & AY624253 & N/A \\
\hline Diplodia scrobiculata & CBS 109944 & DQ458899 & DQ458884 & EU673268 \\
\hline Diplodia scrobiculata & CBS 113423 & DQ458900 & DQ458885 & EU673267 \\
\hline Diplodia seriata & CBS 112555 & AY259094 & AY573220 & AY928050 \\
\hline Diplodia seriata & CBS 119049 & DQ458889 & DQ458874 & EU673266 \\
\hline Diplodia subglobosa & CBS 124133 & $G Q 923856$ & $G Q 923824$ & $N / A$ \\
\hline Diplodia tsugae & CBS 418.64 & DQ458888 & DQ458873 & DQ377867 \\
\hline Dothiorella acacicola & CBS 141295 & $K X 228269$ & $K X 228376$ & $K X 228320$ \\
\hline Dothiorella americana & CBS 128309 & HQ288218 & HQ288262 & N/A \\
\hline Dothiorella americana & CBS 128310 & HQ288219 & HQ288263 & N/A \\
\hline Dothiorella brevicollis & CBS 130411 & JQ239403 & JQ239390 & JQ239416 \\
\hline Dothiorella californica & CBS 141587 & KX357188 & $K X 357211$ & $N / A$ \\
\hline Dothiorella capri-amissi & $C M W 25403$ & $E U 101323$ & $E U 101368$ & $N / A$ \\
\hline Dothiorella capri-amissi & $C M W 25404$ & EU101324 & EU101369 & N/A \\
\hline Dothiorella casuarini & CBS 120688 & DQ846773 & DQ875331 & N/A \\
\hline Dothiorella casuarini & CBS 120690 & DQ846774 & DQ875333 & N/A \\
\hline Dothiorella dulcispinae & CBS 130413 & JQ239400 & JQ239387 & JQ239413 \\
\hline Dothiorella iberica & CBS 115041 & AY573202 & AY573222 & AY928053 \\
\hline Dothiorella iberica & CBS 113188 & AY573198 & EU673278 & EU673230 \\
\hline Dothiorella iranica & IRAN1587C & KC898231 & КC898214 & $N / A$ \\
\hline Dothiorella longicollis & CBS 122068 & EU144054 & EU144069 & N/A \\
\hline Dothiorella longicollis & CBS 122066 & EU144052 & EU144067 & N/A \\
\hline Dothiorella moneti & MUCC505 & EF591920 & EF591971 & EF591937 \\
\hline Dothiorella moneti & MUCC 507 & EF591922 & EF591973 & EF591939 \\
\hline Dothiorella neclivorem & DAR80992 & KJ573643 & KJ573640 & $N / A$ \\
\hline Dothiorella oblonga & CMW 25407 & $E U 101300$ & EU101345 & $N / A$ \\
\hline Dothiorella omnivora & CBS 140349 & KP205497 & KP205470 & $N / A$ \\
\hline Dothiorella omnivora & CBS 188.87 & EU673316 & EU673283 & $N / A$ \\
\hline Dothiorella parva & IRAN1579C & KC898234 & КС898217 & $N / A$ \\
\hline Dothiorella parva & IRAN1585C & КС898235 & КС898218 & $N / A$ \\
\hline
\end{tabular}




\begin{tabular}{|c|c|c|c|c|}
\hline Species & Cultures & ITS & tef1- $\alpha$ & LSU \\
\hline Dothiorella pretoriensis & CBS 130404 & JQ239405 & JQ239392 & JQ239418 \\
\hline Dothiorella pretoriensis & CBS 130403 & JQ239406 & JQ239393 & JQ239419 \\
\hline Dothiorella prunicola & CBS 124723 & EU673313 & EU673280 & EU673232 \\
\hline Dothiorella rhamni & MFLUCC 14-0902 & $K U 246381$ & $N / A$ & KU246382 \\
\hline Dothiorella santali & MUCC 509 & EF591924 & EF591975 & EF591941 \\
\hline Dothiorella santali & MUCC 508 & EF591923 & EF591974 & EF591940 \\
\hline Dothiorella sarmentorum & IMI 63581b & AY573212 & AY573235 & AY928052 \\
\hline Dothiorella sarmentorum & CBS 115038 & AY573206 & AY573223 & DQ377860 \\
\hline Dothiorella sempervirentis & IRAN1583C & КС898236 & КС898219 & $N / A$ \\
\hline Dothiorella sempervirentis & IRAN1581C & КС898237 & KC898220 & N/A \\
\hline Dothiorella striata & ICMP16824 & EU673320 & EU673287 & EU673240 \\
\hline Dothiorella striata & ICMP16819 & EU673320 & EU673287 & EU673239 \\
\hline Dothiorella symphoricarposicola & MFULCC 13-0497 & KJ742378 & KJ742381 & N/A \\
\hline Dothiorella symphoricarposicola & MFLUCC 13-0196 & KU234782 & KU234796 & N/A \\
\hline Dothiorella tectonae & MFLUCC12-0382 & KM396899 & KM409637 & $N / A$ \\
\hline Dothiorella thailandica & CBS 133991 & JX646796 & JX646861 & JX646813 \\
\hline Dothiorella thripsita & BRIP 51876 & FJ824738 & KJ573639 & $N / A$ \\
\hline Dothiorella ulmacea & CBS 138855 & KR611881 & KR611910 & KR611899 \\
\hline Dothiorella uruguayensis & CBS 124908 & EU080923 & EU863180 & N/A \\
\hline Dothiorella vidmadera & DAR78992 & $E U 768874$ & $E U 768881$ & $N / A$ \\
\hline Dothiorella vidmadera & DAR78993 & EU768876 & EU768882 & N/A \\
\hline Dothiorella vinea-gemmae & DAR81012 & KJ573644 & KJ573641 & $N / A$ \\
\hline Endomelanconiopsis endophytica & CBS 120397 & EU683656 & EU683637 & EU683629 \\
\hline Endomelanconiopsis endophytica & CBS 122550 & EU683664 & EU683645 & EU683634 \\
\hline Endomelanconiopsis microspora & CBS 353.97 & EU683655 & EU683636 & EU683628 \\
\hline Eutiarosporella africana & CBS 133854 & KC769956 & KC769852 & KC769990 \\
\hline Eutiarosporella africana & CBS 135850 & КС769957 & КС769853 & КС769991 \\
\hline Eutiarosporella dactylidis & MFLUCC 13-0276 & KM978944 & KP031694 & KM978949 \\
\hline Eutiarosporella dactylidis & MFLUCC 15-0915 & $K U 246378$ & N/A & KU246380 \\
\hline Eutiarosporella darliae & DAR 82491 & KP309793 & KP309805 & $N / A$ \\
\hline Eutiarosporella darliae & DAR 82493 & КР309786 & КР309807 & N/A \\
\hline Eutiarosporella pseudodarliae & DAR 82489 & KP309796 & KP309797 & $N / A$ \\
\hline Eutiarosporella pseudodarliae & DAR 82490 & KР309794 & KP309800 & $N / A$ \\
\hline Eutiarosporella tritici & CBS 118719 & KC769961 & KF531809 & $D Q 377941$ \\
\hline Eutiarosporella tritici-australis & DAR 82485 & KP309788 & KP309799 & $N / A$ \\
\hline Eutiarosporella tritici-australis & DAR 82486 & КР309787 & КР309804 & N/A \\
\hline Eutiarosporella urbis-rosarum & CBS 130405 & $J Q 239407$ & $J Q 239394$ & $J Q 239420$ \\
\hline Eutiarosporella urbis-rosarum & CBS 130406 & $J Q 239408$ & $J Q 239395$ & $J Q 239421$ \\
\hline Lasiodiplodia avicenniae & $C M W 41467$ & КP860835 & КР860680 & N/A \\
\hline Lasiodiplodia avicenniae & LAS199 & KU587957 & KU587946 & N/A \\
\hline Lasiodiplodia brasiliense & CMM 4015 & $J X 464063$ & $J X 464049$ & $N / A$ \\
\hline Lasiodiplodia brasiliense & $C M M 2185$ & KC484800 & KC481530 & $N / A$ \\
\hline Lasiodiplodia bruguierae & $C M W 41470$ & KP860832 & KP860677 & $N / A$ \\
\hline Lasiodiplodia bruguierae & CMW41614 & KP860833 & KP860678 & $N / A$ \\
\hline Lasiodiplodia caatinguensis & CMM1325 & $K T 154760$ & KT008006 & $N / A$ \\
\hline Lasiodiplodia caatinguensis & IBL352 & KT154759 & KT154753 & N/A \\
\hline Lasiodiplodia citricola & CBS 124707 & GU945354 & GU945340 & N/A \\
\hline Lasiodiplodia citricola & CBS 124706 & GU945353 & GU945339 & N/A \\
\hline Lasiodiplodia crassispora & CBS 118741 & DQ103550 & EU673303 & DQ377901 \\
\hline Lasiodiplodia crassispora & WAC 12534 & DQ103551 & DQ103558 & N/A \\
\hline Lasiodiplodia crassispora & CBS 110492 & EF622086 & EF622066 & N/A \\
\hline Lasiodiplodia euphorbicola & СММЗ609 & KF 234543 & KF226689 & N/A \\
\hline Lasiodiplodia euphorbicola & IBL329 & KT247490 & KT247492 & N/A \\
\hline
\end{tabular}




\begin{tabular}{|c|c|c|c|c|}
\hline Species & Cultures & ITS & tef1- $\alpha$ & LSU \\
\hline Lasiodiplodia exigua & CBS 137785 & KJ638317 & KJ638336 & $N / A$ \\
\hline Lasiodiplodia exigua & BL184 & KJ638318 & KJ638337 & N/A \\
\hline Lasiodiplodia gilanensis & CBS 124704 & GU945351 & GU945342 & $N / A$ \\
\hline Lasiodiplodia gilanensis & CBS 124705 & GU945352 & GU945341 & N/A \\
\hline Lasiodiplodia gonubiensis & CBS 115812 & AY639595 & DQ103566 & N/A \\
\hline Lasiodiplodia gonubiensis & CBS 116355 & AY639594 & DQ103567 & N/A \\
\hline Lasiodiplodia gravistriata & CMM 4564 & KT250949 & KT250950 & $N / A$ \\
\hline Lasiodiplodia gravistriata & $C M M 4565$ & KT250947 & KT266812 & $N / A$ \\
\hline Lasiodiplodia hormozganensis & CBS 124709 & GU945355 & GU945343 & $N / A$ \\
\hline Lasiodiplodia hormozganensis & CBS 124708 & GU945356 & GU945344 & N/A \\
\hline Lasiodiplodia iraniensis & CBS 124710 & GU945346 & GU945334 & $N / A$ \\
\hline Lasiodiplodia iraniensis & CBS 124711 & GU945347 & GU945335 & N/A \\
\hline Lasiodiplodia laeliocattleyae & CBS 167.28 & KU507487 & KU507454 & $N / A$ \\
\hline Lasiodiplodia lignicola & CBS 134112 & JX646797 & KU887003 & N/A \\
\hline Lasiodiplodia macrospora & CMM3833 & KF234557 & KF226718 & N/A \\
\hline Lasiodiplodia mahajangana & CBS 124927 & FJ900597 & FJ900643 & N/A \\
\hline Lasiodiplodia mahajangana & CBS 124925 & FJ900595 & FJ900641 & N/A \\
\hline Lasiodiplodia margaritacea & CBS 122519 & EU144050 & EU144065 & N/A \\
\hline Lasiodiplodia margaritacea & CBS 122065 & EU144051 & EU144066 & N/A \\
\hline Lasiodiplodia mediterranea & CBS 137783 & KJ638312 & KJ638331 & $N / A$ \\
\hline Lasiodiplodia mediterranea & ALG36 & KJ638314 & KJ638333 & N/A \\
\hline Lasiodiplodia missouriana & CBS 128311 & HQ288225 & HQ288267 & N/A \\
\hline Lasiodiplodia missouriana & CBS 128312 & HQ288226 & HQ288268 & N/A \\
\hline Lasiodiplodia parva & CBS 456.78 & EF622083 & EF622063 & KF766362 \\
\hline Lasiodiplodia parva & CBS 494.78 & EF622084 & EF622064 & N/A \\
\hline Lasiodiplodia plurivora & CBS 120832 & EF445362 & EF445395 & $N / A$ \\
\hline Lasiodiplodia plurivora & CBS 121103 & AY343482 & EF445396 & N/A \\
\hline Lasiodiplodia pontae & CMM1277 & KT151794 & KT151791 & $N / A$ \\
\hline Lasiodiplodia pseudotheobromae & CBS 116459 & EF622077 & EF622057 & N/A \\
\hline Lasiodiplodia pseudotheobromae & CBS 447.62 & EF622081 & EF622060 & N/A \\
\hline Lasiodiplodia pyriformis & CMW 25414 & EU101307 & EU101352 & $N / A$ \\
\hline Lasiodiplodia pyriformis & $C M W 25415$ & EU101308 & EU101353 & N/A \\
\hline Lasiodiplodia rubropurpurea & CBS 118740 & DQ103553 & EU673304 & N/A \\
\hline Lasiodiplodia rubropurpurea & WAC 12536 & DQ103554 & DQ103572 & N/A \\
\hline Lasiodiplodia subglobosa & CMM3872 & KF234558 & KF226721 & $N / A$ \\
\hline Lasiodiplodia subglobosa & CMM 4046 & $K F 234560$ & KF226723 & N/A \\
\hline Lasiodiplodia thailandica & CPC 22795 & KJ193637 & KJ193681 & $N / A$ \\
\hline Lasiodiplodia theobromae & CBS 164.96 & AY640255 & AY640258 & EU673253 \\
\hline Lasiodiplodia theobromae & CBS124.13 & DQ458890 & DQ458875 & N/A \\
\hline Lasiodiplodia theobromae & CBS 111530 & EF622074 & EF622054 & N/A \\
\hline Lasiodiplodia theobromae & CAA 006 & DQ458891 & DQ458876 & N/A \\
\hline Lasiodiplodia venezuelensis & CBS 118739 & DQ103547 & EU673305 & N/A \\
\hline Lasiodiplodia venezuelensis & WAC 12540 & DQ103548 & DQ103569 & N/A \\
\hline Lasiodiplodia viticola & CBS 128313 & HQ288227 & HQ288269 & N/A \\
\hline Lasiodiplodia viticola & CBS 128315 & HQ288228 & HQ288270 & N/A \\
\hline Macrophomina phaseolina & CBS 227.33 & KF951627 & KF952000 & DQ377906 \\
\hline Macrophomina phaseolina & CBS 162.25 & KF531826 & KF951996 & DQ377905 \\
\hline Marasasiomyces karoo & CBS 118718 & KF531828 & KF531807 & $D Q 377939$ \\
\hline Mucoharknessia cortaderiae & СРC 19974 & KM108374 & $N / A$ & KM108401 \\
\hline Mucoharknessia cortaderiae & CPC 22208 & KM108375 & N/A & KM108402 \\
\hline Mucoharknessia anthoxanthii & MFLUCC 15-0904 & KU246377 & $N / A$ & KU246379 \\
\hline Neodeightonia licuriensis & COAD1780 & KP165429 & KP165430 & $N / A$ \\
\hline Neodeightonia palmicola & MFLUCC 10-0822 & HQ199221 & N/A & HQ199222 \\
\hline
\end{tabular}




\begin{tabular}{|c|c|c|c|c|}
\hline Species & Cultures & ITS & tef1- $\alpha$ & LSU \\
\hline Neodeightonia palmicola & MFLUCC 10-0823 & HQ199224 & N/A & HQ199225 \\
\hline Neodeightonia phoenicum & CBS 122528 & EU673340 & EU673309 & EU673261 \\
\hline Neodeightonia phoenicum & CBS 169.34 & EU673338 & EU673307 & EU673259 \\
\hline Neodeightonia rattanica & MFLUCC 15-0712 & KX646357 & $K X 646360$ & $K X 646352$ \\
\hline Neodeightonia rattanica & MFLUCC 15-0313 & KХ646358 & KX646361 & KX646353 \\
\hline Neodeightonia rattanicola & MFLUCC 15-0319 & KX646359 & $K X 646362$ & $K X 646354$ \\
\hline Neodeightonia subglobosa & CBS 448.91 & EU673337 & EU673306 & DQ377866 \\
\hline Neodeightonia subglobosa & MFLUCC 11-0163 & JX646794 & JX646859 & JX646811 \\
\hline Neofusicoccum algeriense & CBS 137504 & KJ657702 & KJ657715 & $N / A$ \\
\hline Neofusicoccum algeriense & $A L G 9$ & KJ657704 & KJ657721 & $N / A$ \\
\hline Neofusicoccum andinum & CBS 117453 & AY693976 & AY693977 & $N / A$ \\
\hline Neofusicoccum andinum & CBS 117452 & DQ306263 & DQ306264 & N/A \\
\hline Neofusicoccum arbuti & CBS 116131 & AY819720 & N/A & N/A \\
\hline Neofusicoccum arbuti & CBS 117090 & AY819724 & KF531791 & N/A \\
\hline Neofusicoccum australe & CMW 6837 & AY339262 & AY339270 & KF766367 \\
\hline Neofusicoccum australe & CMW 6853 & AY339263 & AY339271 & N/A \\
\hline Neofusicoccum batangarum & CBS 124924 & FJ900607 & FJ900653 & N/A \\
\hline Neofusicoccum batangarum & CBS 124923 & FJ900608 & FJ900654 & N/A \\
\hline Neofusicoccum braziliense & CMM1338 & $J X 513630$ & $J X 513610$ & $N / A$ \\
\hline Neofusicoccum cordaticola & CBS 123634 & EU821898 & EU821868 & $N / A$ \\
\hline Neofusicoccum cordaticola & CBS 123635 & EU821903 & EU821873 & N/A \\
\hline Neofusicoccum cryptoaustrale & $C M W 23785$ & FJ752742 & FJ752713 & $N / A$ \\
\hline Neofusicoccum eucalypticola & CBS 115679 & AY615141 & AY615133 & N/A \\
\hline Neofusicoccum eucalypticola & CBS 115766 & AY615143 & AY615135 & N/A \\
\hline Neofusicoccum eucalyptorum & CBS 115791 & AF283686 & AY236891 & N/A \\
\hline Neofusicoccum eucalyptorum & CMW 10126 & AF283687 & AY236892 & N/A \\
\hline Neofusicoccum grevilleae & CBS 129518 & JF951137 & N/A & N/A \\
\hline Neofusicoccum hellenicum & CERC1947 & KP217053 & KP217061 & $N / A$ \\
\hline Neofusicoccum kwambonambiense & CBS 123639 & EU821900 & EU821870 & N/A \\
\hline Neofusicoccum kwambonambiense & CBS 123641 & EU821919 & EU821889 & N/A \\
\hline Neofusicoccum lumnitzerae & $C M W 41469$ & KP860881 & KP860724 & $N / A$ \\
\hline Neofusicoccum lumnitzerae & CMW41228 & KP860882 & KP860725 & $N / A$ \\
\hline Neofusicoccum luteum & CBS 110299 & AY259091 & AY573217 & AY928043 \\
\hline Neofusicoccum luteum & CBS 110497 & EU673311 & EU673277 & N/A \\
\hline Neofusicoccum macroclavatum & CBS 118223 & DQ093196 & DQ093217 & N/A \\
\hline Neofusicoccum macroclavatum & WAC 12446 & DQ093197 & DQ093218 & $\mathrm{N} / \mathrm{A}$ \\
\hline Neofusicoccum mangiferae & CBS 118531 & AY615185 & DQ093221 & N/A \\
\hline Neofusicoccum mangiferae & CBS 118532 & AY615186 & DQ093220 & $\mathrm{N} / \mathrm{A}$ \\
\hline Neofusicoccum mangroviorum & $C M W 41365$ & KP860859 & KP860702 & $N / A$ \\
\hline Neofusicoccum mangroviorum & $C M W 42481$ & KP860848 & KP860692 & N/A \\
\hline Neofusicoccum mediterraneum & CBS 121718 & GU251176 & GU251308 & N/A \\
\hline Neofusicoccum mediterraneum & CBS 121558 & GU799463 & GU799462 & N/A \\
\hline Neofusicoccum nonquaesitum & CBS 126655 & GU251163 & GU251295 & N/A \\
\hline Neofusicoccum nonquaesitum & PD 301 & GU251164 & GU251296 & N/A \\
\hline Neofusicoccum occulatum & CBS 128008 & EU301030 & EU339509 & N/A \\
\hline Neofusicoccum occulatum & MUCC 286 & EU736947 & EU339511 & N/A \\
\hline Neofusicoccum parvum & CMW 9081 & AY236943 & AY236888 & AY928045 \\
\hline Neofusicoccum parvum & CBS 110301 & AY259098 & AY573221 & N/A \\
\hline Neofusicoccum pennatisporum & MUCC 510 & EF591925 & EF591976 & N/A \\
\hline Neofusicoccum protearum & STE-U 4361 & AF196295 & N/A & N/A \\
\hline Neofusicoccum protearum & STE-U 1775 & AF452539 & N/A & N/A \\
\hline Neofusicoccum ribis & CBS 115475 & AY236935 & AY236877 & N/A \\
\hline Neofusicoccum ribis & CBS 121.26 & AF241177 & AY236879 & N/A \\
\hline
\end{tabular}




\begin{tabular}{|c|c|c|c|c|}
\hline Species & Cultures & ITS & tef1- $\alpha$ & LSU \\
\hline Neofusicoccum umdonicola & CBS 123645 & EU821904 & EU821874 & N/A \\
\hline Neofusicoccum umdonicola & CBS 123646 & EU821905 & EU821875 & N/A \\
\hline Neofusicoccum ursorum & $C M W 24480$ & FJ752746 & FJ752709 & $N / A$ \\
\hline Neofusicoccum viticlavatum & CBS 112878 & AY343381 & AY343342 & N/A \\
\hline Neofusicoccum viticlavatum & CBS 112977 & AY343380 & AY343341 & N/A \\
\hline Neofusicoccum vitifusiforme & CBS 110887 & AY343383 & AY343343 & N/A \\
\hline Neofusicoccum vitifusiforme & CBS 110880 & AY343382 & AY343344 & N/A \\
\hline Neoscytalidium dimidiatum & CBS 145.78 & KF531816 & KF531795 & $D Q 377922$ \\
\hline Neoscytalidium dimidiatum & CBS 499.66 & KF531820 & KF531798 & DQ377925 \\
\hline Neoscytalidium dimidiatum & CBS 251.49 & KF531819 & KF531797 & DQ377923 \\
\hline Neoscytalidium dimidiatum & UTHSCSA DI 14-340 & KM357894 & N/A & KM357895 \\
\hline Neoscytalidium dimidiatum & IP127881 & AY819727 & N/A & DQ377925 \\
\hline Neoscytalidium dimidiatum & CBS 135275 & KF571862 & N/A & N/A \\
\hline Neoscytalidium novaehollandiae & CBS 122071 & EF585540 & EF585580 & KF766374 \\
\hline Neoscytalidium novaehollandiae & CBS 122610 & EF585536 & EF585578 & N/A \\
\hline Neoscytalidium orchidacearum & MFLUCC 12-0533 & KU179865 & $N / A$ & KU179864 \\
\hline Phaeobotryon cupressi & CBS 124700 & FJ919672 & FJ919661 & N/A \\
\hline Phaeobotryon cupressi & IRAN $1458 \mathrm{C}$ & FJ919671 & FJ919660 & N/A \\
\hline Phaeobotryon mamane & CBS 122980 & EU673332 & EU673298 & EU673248 \\
\hline Phaeobotryon mamane & СРC 12442 & EU673333 & EU673299 & DQ377899 \\
\hline Phaeobotryon negundinis & MFLUCC 15-0436 & $K U 820970$ & KU853997 & KU820971 \\
\hline Phaeobotryon negundinis & CAA 797 & KХ061513 & КХ061507 & $N / A$ \\
\hline Phaeobotryon rhois & CFCC 89662 & KM030584 & KM030598 & KM030591 \\
\hline Phaeobotryon rhois & CFCC 89663 & KM030585 & KM030599 & KM030592 \\
\hline Pseudofusicoccum adansoniae & CBS 122055 & EF585523 & EF585571 & N/A \\
\hline Pseudofusicoccum adansoniae & WAC 12689 & EF585534 & EF585567 & EF585554 \\
\hline Pseudofusicoccum ardesiacum & CBS 122062 & EU144060 & EU144075 & N/A \\
\hline Pseudofusicoccum ardesiacum & WAC 13294 & GU172405 & GU172437 & N/A \\
\hline Pseudofusicoccum artocarpi & CPC 22796 & KM006452 & KM006483 & N/A \\
\hline Pseudofusicoccum kimberleyensis & CBS 122058 & EU144057 & EU144072 & N/A \\
\hline Pseudofusicoccum kimberleyensis & CBS 122059 & EU144056 & EU144071 & N/A \\
\hline Pseudofusicoccum olivaceum & CBS 124939 & FJ888459 & FJ888437 & N/A \\
\hline Pseudofusicoccum olivaceum & CBS 124940 & FJ888462 & FJ888438 & N/A \\
\hline Pseudofusicoccum stromaticum & CBS 117448 & AY693974 & AY693975 & DQ377931 \\
\hline Pseudofusicoccum stromaticum & CBS 117449 & DQ436935 & DQ436936 & DQ377932 \\
\hline Pseudofusicoccum violaceum & CBS 124936 & FJ888474 & FJ888442 & N/A \\
\hline Pseudofusicoccum violaceum & CBS 124937 & FJ888458 & FJ888440 & N/A \\
\hline Sakireeta madreeya & CBS 532.76 & KC769960 & KM108427 & $D Q 377940$ \\
\hline Sardiniella urbana & CBS 141580 & KX379674 & $K X 379675$ & $K X 379676$ \\
\hline Sardiniella urbana & BL180 & KХ379677 & KX379678 & KX379679 \\
\hline Sardiniella urbana & BL181 & KХ379680 & KX379681 & KX379682 \\
\hline Spencermartinsia alpina & CGMCC 3.18001 & $K X 499645$ & $K X 499651$ & $N / A$ \\
\hline Spencermartinsia citricola & ICMP16828 & EU673323 & EU673290 & $N / A$ \\
\hline Spencermartinsia citricola & CMP16827 & EU673322 & EU673289 & N/A \\
\hline Spencermartinsia mangiferae & IRAN1584C & KC898221 & КС898204 & N/A \\
\hline Spencermartinsia mangiferae & IRAN1545C & КС898223 & КС898206 & $N / A$ \\
\hline Spencermartinsia plurivora & IRAN1557C & KC898225 & KC898208 & $N / A$ \\
\hline Spencermartinsia plurivora & IRAN1537C & КС898226 & КС898209 & $N / A$ \\
\hline Spencermartinsia rosulata & CBS 121760 & $E U 101290$ & EU101335 & N/A \\
\hline Spencermartinsia rosulata & $C M W 25392$ & EU101293 & EU101338 & $N / A$ \\
\hline Spencermartinsia viticola & CBS 117009 & AY905554 & AY905559 & DQ377873 \\
\hline Spencermartinsia westrale & DAR80529 & HМ009376 & HM800511 & $N / A$ \\
\hline Spencermartinsia westrale & DAR80530 & HМ009377 & HM800512 & $N / A$ \\
\hline Spencermartinsia yunnana & CGMCC 3.17999 & $K X 499643$ & KX499649 & $N / A$ \\
\hline
\end{tabular}




\begin{tabular}{|c|c|c|c|c|}
\hline Species & Cultures & ITS & tef1- $\alpha$ & LSU \\
\hline Spencermartinsia yunnana & CGMCC 3.18000 & $K X 499644$ & $K X 499650$ & $N / A$ \\
\hline Sphaeropsis citrigena & ICMP 16812 & EU673328 & EU673294 & EU673246 \\
\hline Sphaeropsis citrigena & ICMP 16818 & EU673329 & EU673295 & EU673247 \\
\hline Sphaeropsis eucalypticola & CBS 133993 & JX646802 & JX646867 & JX646819 \\
\hline Sphaeropsis eucalypticola & MFLUCC 11-0654 & JX646803 & JX646868 & JX646820 \\
\hline Sphaeropsis porosa & CBS 110496 & AY343379 & AY343340 & DQ377894 \\
\hline Sphaeropsis porosa & CBS 110574 & AY343378 & AY343339 & DQ377895 \\
\hline Sphaeropsis variabilis & $C M W 25420$ & EU101313 & $E U 101358$ & $N / A$ \\
\hline Sphaeropsis variabilis & $C M W 25421$ & EU101314 & EU101359 & N/A \\
\hline Sphaeropsis visci & CBS 122526 & EU673324 & EU673292 & N/A \\
\hline Sphaeropsis visci & CBS 186.97 & EU673325 & EU673293 & DQ377868 \\
\hline Tiarosporella paludosa & CPC 22701 & KM108378 & $N / A$ & KM108404 \\
\hline Tiarosporella paludosa & CPC 22702 & KM108379 & $N / A$ & KM108405 \\
\hline \multicolumn{5}{|l|}{ Aplosporellaceae } \\
\hline Aplosporella javeedii & CMW 38166 & KC769939 & КC769847 & KC769980 \\
\hline Aplosporella prunicola & CBS 121167 & KF766147 & N/A & JX681071 \\
\hline Aplosporella yalgorensis & MUCC 511 & EF591927 & EF591978 & EF591944 \\
\hline Bagnisiella examinans & CBS 551.66 & KF766148 & GU349056 & KF766316 \\
\hline \multicolumn{5}{|l|}{ Melanopsaceae } \\
\hline Melanops sp. & CBS 118.39 & FJ824771 & FJ824776 & DQ377856 \\
\hline Melanops tulasnei & CBS 116805 & FJ824769 & FJ824774 & FJ824764 \\
\hline \multicolumn{5}{|l|}{ Phyllostictaceae } \\
\hline Phyllosticta citricarpa & CBS 102374 & FJ824767 & FJ538376 & DQ377877 \\
\hline Phyllosticta minima & CBS 111635 & KF766215 & KF766433 & EU754194 \\
\hline Phyllosticta parthenocissi & CBS 111645 & FJ824766 & EU683653 & DQ377876 \\
\hline Phyllosticta philoprina & CBS 616.72 & KF766171 & KF766402 & KF766341 \\
\hline Phyllosticta podocarpi & CBS 111647 & KF766217 & KF766434 & KF766383 \\
\hline \multicolumn{5}{|l|}{ Planistromellaceae } \\
\hline Kellermania crassispora & CBS 131714 & KF766175 & KF766406 & KF766345 \\
\hline Kellermania dasylirionis & CBS 131715 & KF766177 & KF766408 & KF766347 \\
\hline Kellermania macrospora & CBS 131716 & KF766178 & KF766409 & KF766348 \\
\hline Kellermania nolinae & CBS 131717 & KF766180 & KF766411 & KF766350 \\
\hline Kellermania plurilocularis & CBS 131719 & KF766181 & KF766412 & KF766351 \\
\hline Kellermania yuccifoliorum & CBS 131726 & KF766185 & KF766416 & KF766355 \\
\hline \multicolumn{5}{|l|}{ Saccharataceae } \\
\hline Saccharata capensis & CBS 122693 & KF766224 & EU552095 & KF766390 \\
\hline Saccharata kirstenboschensis & CBS 123537 & KF766225 & N/A & FJ372409 \\
\hline Saccharata proteae & CBS 115206 & KF766226 & KF766438 & DQ377882 \\
\hline \multicolumn{5}{|l|}{ Septorioidaceae } \\
\hline Septorioides pini-thunbergii & CBS 473.91 & KF251243 & N/A & KF251746 \\
\hline Septorioides strobi & CBS141443 & KT884699 & KT884713 & KT884685 \\
\hline Septorioides strobi & CBS141444 & KT884700 & KT884714 & КT884686 \\
\hline Septorioides strobi & CBS141445 & KT884702 & KT884716 & КT884688 \\
\hline Septorioides strobi & V-3-11-1 & KT884703 & KT884717 & КT884689 \\
\hline \multicolumn{5}{|l|}{ Outgroup } \\
\hline Lecanosticta acicola & LNPV 252 & JX901755 & JX901639 & JX901844 \\
\hline
\end{tabular}

of a random starting tree and 1000 ML bootstrap replicates. The search strategy was set to rapid bootstrapping with one thousand non-parametric bootstrapping iterations using the general time reversible model (GTR) with a discrete gamma distribution. The best scoring trees were selected with a final likelihood values. 
Posterior probabilities (PP) were determined by Markov Chain Monte Carlo sampling (BMCMC) in MrBayes v. 3.0b4 (Ronquist \& Huelsenbeck 2003). MrModeltest v. 2.3 (Nylander 2004) was used to carry out statistical selection of best-fit model of nucleotide substitution and was incorporated into the analysis. Six simultaneous Markov chains were run for 1000000 generations and trees were sampled every $100^{\text {th }}$ generation. The 2000 trees representing the burn-in phase of the analyses, were discarded and the remaining 8000 trees used for calculation of posterior probabilities (PP) in the majority rule consensus tree. The strains used in this study are listed in Table 1 with details of type cultures and sequence data.

\section{Results and Discussion}

A complete list of all genera and species in the Botryosphaeriaceae that are known from culture is provided in Table 1. Where possible the ex-type isolate together with one further isolate are included. Also included in Table 1 are representative isolates of other familes in the Botryosphaeriales.

\section{The family Botryosphaeriaceae}

Presently 23 genera are included the family (Fig. 1). Some doubts remain about the limits of the family, especially regarding the position of Pseudofusicoccum. Slippers et al (2013) considered that Pseudofusicoccum lies in a separate clade at the base of the family while Phillips et al. (2013) placed it as sister to Endomelanconiopsis within Botryosphaeriaceae. Although in Fig. 1 we show it to be sister to Phyllosticta, and thus within Phyllostictaceae, this tree is based on only 3 loci and thus is not as robust as the trees of Slippers et al (2013) or Phillips et al. (2013). Therefore at this stage we consider that Pseudofucicoccum is a genus within Botryosphaeriaceae even though its position remains uncertain. Apart from Pseudofusicoccum the positions of the remaining 22 genera are similar to previously published phylogenies of Botryosphaeriaceae.

\section{Genera and species}

1. Alanphillipsia Crous \& M.J. Wingf., Persoonia 31: 197 (2013), MycoBank MB805816.

Alanphillipsia was introduced to accommodate species that are morphologically aplosporella-like but the conidia have a hyaline outer layer (Crous et al. 2013, 2014). Five species are currently known and all have been reported from the Western Cape Province of South Africa. The sexual morph has not been reported for any species.

Type species - Alanphillipsia aloes Crous \& M.J. Wingf. 2013.

\subsection{Alanphillipsia aloeicola Crous, Persoonia 33: 217 (2014), MycoBank MB810590.}

Sexual morph not reported. See Crous et al. (2014) for illustrations and descriptions of asexual morph.

Type - South Africa, Western Cape Province, Clanwilliam, Ramskop, Aloe sp. (Asphodelaceae), Sept. 2013, M.J. Wingfield (holotype CBS H-21978, culture ex-type CBS 138896).

Host - Aloe sp. (Crous et al. 2014).

Distribution - South Africa (Crous et al. 2014).

1.2. Alanphillipsia aloeigena Crous \& M.J. Wingf., Persoonia 31: 199 (2013), MycoBank MB805818.

Sexual morph not reported. See Crous et al. (2013) for illustrations and descriptions of asexual morph.

Type-South Africa, Namakwaland, Goegap Nature Reserve, on leaves of Aloe melanacantha (Asphodelaceae), 26 Sept. 2012, M.J. Wingfield (holotype CBS H-21419, culture ex-type CPC $21286=$ CBS 136408).

Host - Aloe melanocantha (Crous et al. 2013).

Distribution - South Africa (Crous et al. 2013). 


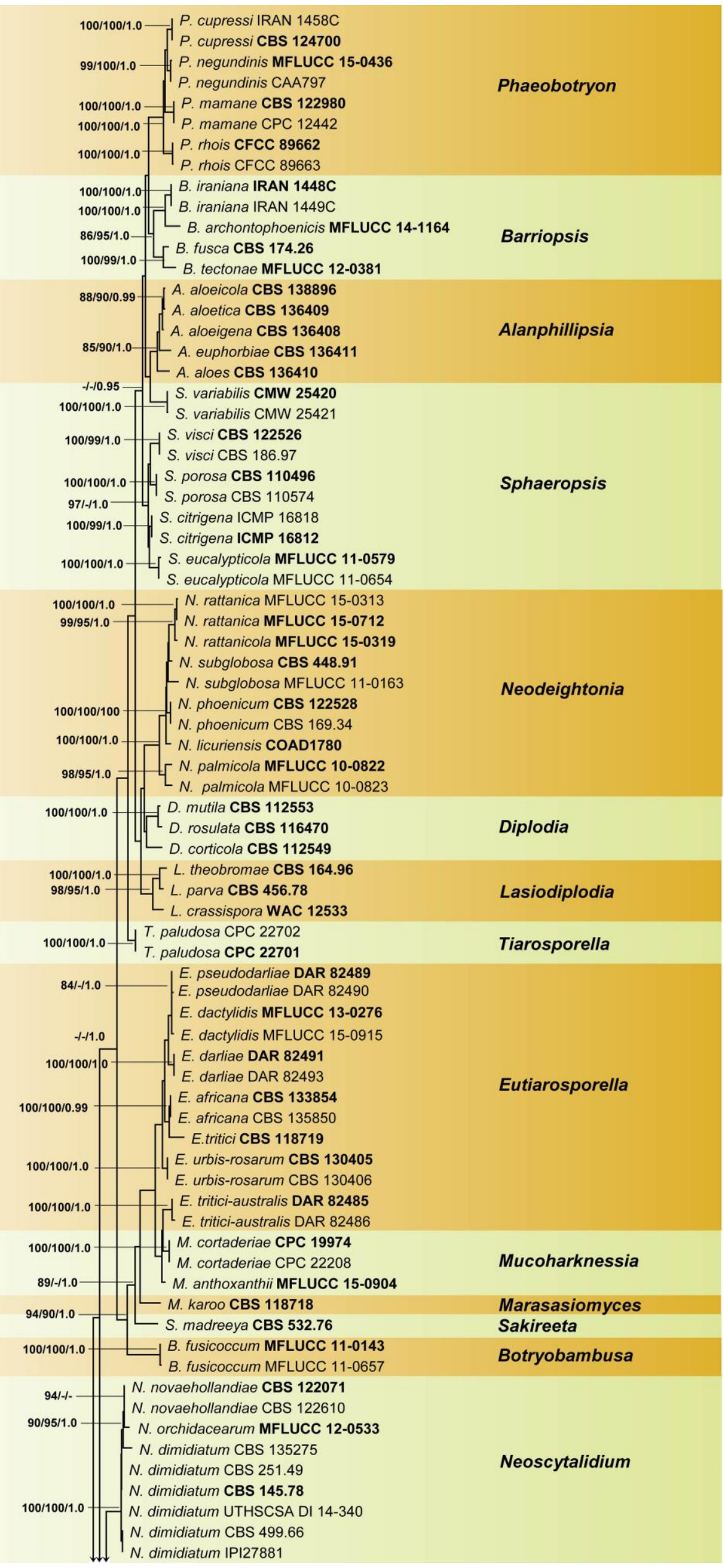




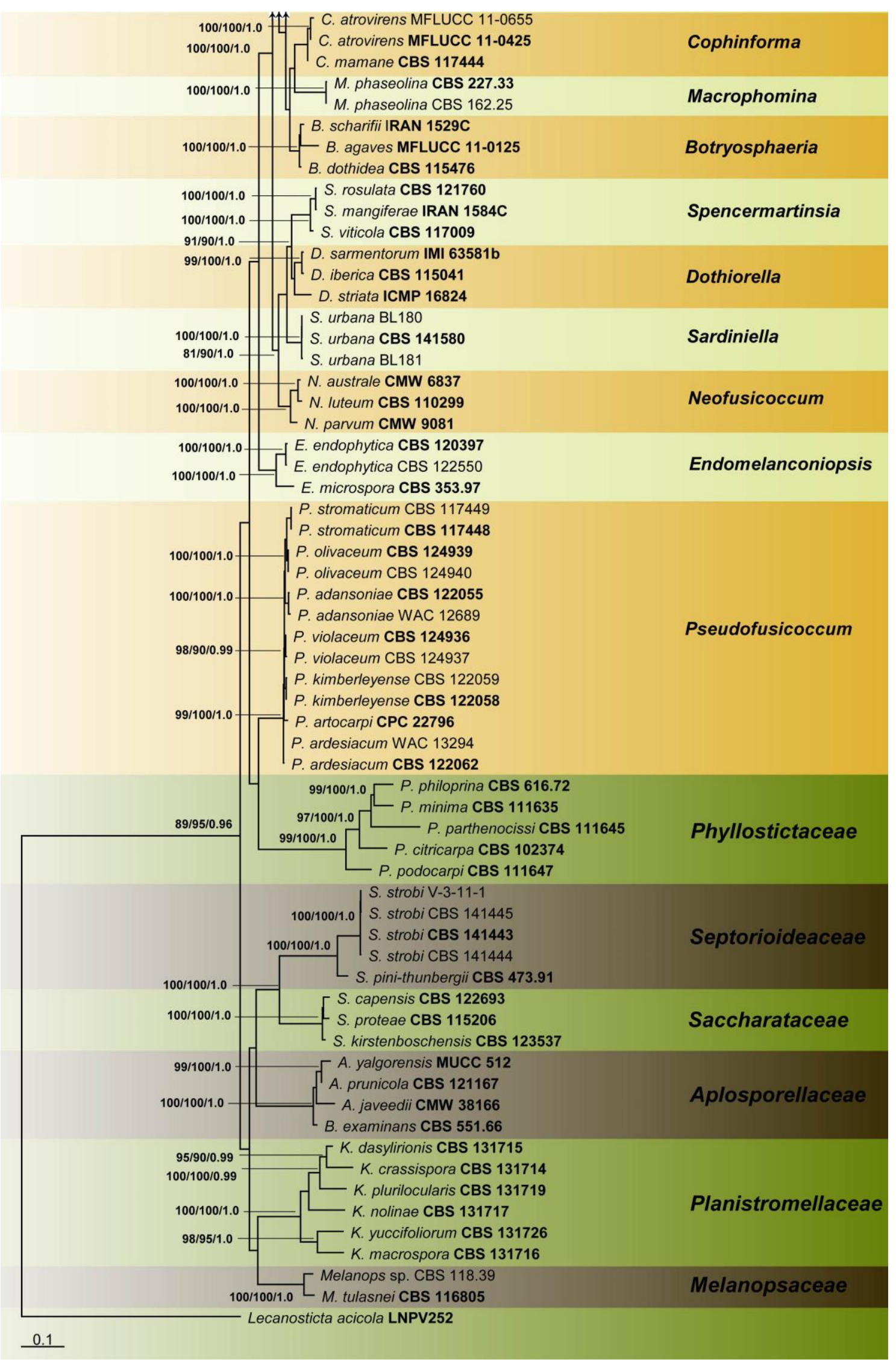

Fig. 1 - Phylogram generated from Maximum Likelihood analysis based on combined ITS, tefl- $\alpha$ and LSU dataset of Botryosphaeriales. Bootstrap support values for maximum likelihood (ML), maximum parsimony (MP) greater than $75 \%$ and Bayesian posterior probabilities above 0.90 are indicated near the nodes. The ex-type strains are in bold and the tree is rooted with Lecanosticta acicola (LNPV252). 
1.3. Alanphillipsia aloes Crous \& M.J. Wingf., Persoonia 31: 197 (2013), MycoBank MB805817. Sexual morph not reported. See Crous et al. (2013) for illustrations and descriptions of asexual morph.

Type - South Africa, Western Cape Province, Clanwilliam, on dark lesions of dying Aloe dichotoma (Asphodelaceae), Sept. 2012, M.J. Wingfield (holotype CBS H-21418, cultures ex-type CPC 21298 = CBS 136410).

Host - Aloe dichotoma (Crous et al. 2013).

Distribution - South Africa (Crous et al. 2013).

1.4. Alanphillipsia aloetica Crous, Persoonia 31: 201 (2013), MycoBank MB805819.

Sexual morph not reported. See Crous et al. (2013) for illustrations and descriptions of asexual morph.

Type - South Africa, Eastern Cape, Uitenhage, on Aloe sp. (Asphodelaceae), 14 July 2012, P.W. Crous (holotype CBS H-21420, culture ex-type CPC 21110, 21109 = CBS 136409).

Host - Aloe sp. (Crous et al. 2013).

Distribution - South Africa (Crous et al. 2013).

1.5. Alanphillipsia euphorbiae Crous \& M.J. Wingf., Persoonia 31: 203 (2013), MycoBank MB805821.

Sexual morph not reported. See Crous et al. (2013) for illustrations and descriptions of asexual morph.

Type - South Africa, Western Cape Province, Kirstenbosch Botanical Garden, on leaves of Euphorbia sp. (Euphorbiaceae), Sept. 2012, M.J. Wingfield (holotype CBS H-21421, culture extype CPC 21629, 21628 = CBS 136411).

Host - Aloe sp. (Crous et al. 2013).

Distribution - South Africa (Crous et al. 2013).

2. Barriopsis A.J.L. Phillips, A. Alves \& Crous, Persoonia 21: 39 (2008), MycoBank MB511712. Barriopsis comprises four species. Sexual and asexual morphs have been reported within the genus. The pale brown, 1-septate conidia that are striate when immature are unique in the Botryosphaeriaceae.

Type species - Barriopsis fusca (N.E. Stevens) A.J.L. Phillips, A. Alves \& Crous 2008.

2.1. Barriopsis archontophoenicis Konta, Boonmee \& K.D. Hyde, 7: 924 (2016), Facesoffunginumber FOF02097.

Sexual morph and asexual morph illustrations and descriptions in Konta et al. (2016a, this volume). This species forms the sexual morph in culture after long periods of incubation (up to 6 months).

Type - Thailand, Chiang Mai Province, Mushroom Research Centre, on Archontophoenix alexandrae (Arecaceae), 15 August 2014, S. Konta (holotype MFLU 15-0015, culture ex-type MFLUCC 14-1164, MUCL 55901).

Host - Archontophoenix alexandrae (Konta et al. 2016a).

Distribution - Thailand (Konta et al. 2016a).

2.2. Barriopsis fusca (N.E. Stevens) A.J.L. Phillips, A. Alves \& Crous, Persoonia 21: 39 (2008), MycoBank MB511713.

Sexual morph illustrations and descriptions are available in Liu et al. (2012) and Phillips et al. (2008, 2013). The only available culture of B. fusca (CBS 174.26, ex-type) has lost its ability to 
sporulate. According to Stevens (1926) the asexual morph is lasiodiplodia-like with hyaline conidia that become dark-brown and septate with irregular longitudinal striations.

Type - Cuba, Herradura, on twigs of Citrus sp. (Rutaceae), 15 Jan. 1925, N.E. Stevens (holotype BPI 599052, culture ex-type CBS 174.26).

Host - Citrus sp. (Stevens 1926).

Distribution - Cuba (Stevens 1926), Florida (Shear 1923).

2.3. Barriopsis iraniana Abdollahz., Zare \& A.J.L. Phillips, Persoonia 23: 4 (2009), MycoBank MB513235.

Sexual morph not reported. See Abdollahzadeh et al. (2009), Phillips et al. (2013) for illustrations and descriptions of asexual morph.

Type - Iran, Hormozgan Province, Minab, Hajikhademi, on twigs of Mangifera indica (Anacardiaceae), 27 Feb. 2007, J. Abdollahzadeh \& A. Javadi (holotype IRAN 13939F, culture extype IRAN 1448C = CBS 124698).

Hosts - Citrus sp., Mangifera indica, Olea sp. (Abdollahzadeh et al. 2009).

Distribution - Iran (Abdollahzadeh et al. 2009).

2.4. Barriopsis tectonae Doilom, L.A. Shuttlew. \& K.D. Hyde, Phytotaxa 176: 84 (2014), MycoBank MB808202.

Sexual morph and asexual morph have been reported. See Doilom et al. (2014) for illustrations and descriptions.

Type - Thailand, Phayao Province: Muang District, on dead branch of Tectona grandis (Lamiaceae), 12 March 2012, M. Doilom (holotype MFLU 14-0024, culture ex-type MFLUCC 12$0381=$ CBS 137786).

Host - Tectona grandis (Doilom et al. 2014).

Distribution - Thailand (Doilom et al. (2014).

3. Botryobambusa Phook., J.K. Liu \& K.D. Hyde, Fungal Diversity 57: 166 (2012), MycoBank MB801313.

Botryobambusa comprises a single species, which is known in both the sexual and asexual morph.

Type species - Botryobambusa fusicoccum Phook., J.K. Liu \& K.D. Hyde 2012.

3.1. Botryobambusa fusicoccum Phook., J.K. Liu \& K.D. Hyde, Fungal Diversity 57: 166 (2012), MycoBank MB801314.

Sexual morph and asexual morph have been reported. See Liu et al. (2012), Phillips et al. (2013) for illustrations and descriptions.

Type - Thailand, Lampang Province, Jae Hom District, Mae Yuag Forestry Plantation, on dead culms of Bambusa sp. (Poaceae), 19 Aug. 2010, R. Phookamsak (holotype MFLU 11-0179, culture ex-type CBS 134113 = MFLUCC 11-0143).

Host - Bambusa sp. (Liu et al. 2012).

Distribution - Thailand (Liu et al. 2012).

4. Botryosphaeria Ces. \& De Not., Comm. Soc. crittog. Ital. 1: 211 (1863), MycoBank MB635.

Type genus of Botryosphaeriaceae. Botryosphaeria comprises ten species (Fig. 2). Sexual and asexual morphs have been reported in the genus.

Type species - Botryosphaeria dothidea (Moug. : Fr.) Ces. \& De Not. 1863. 


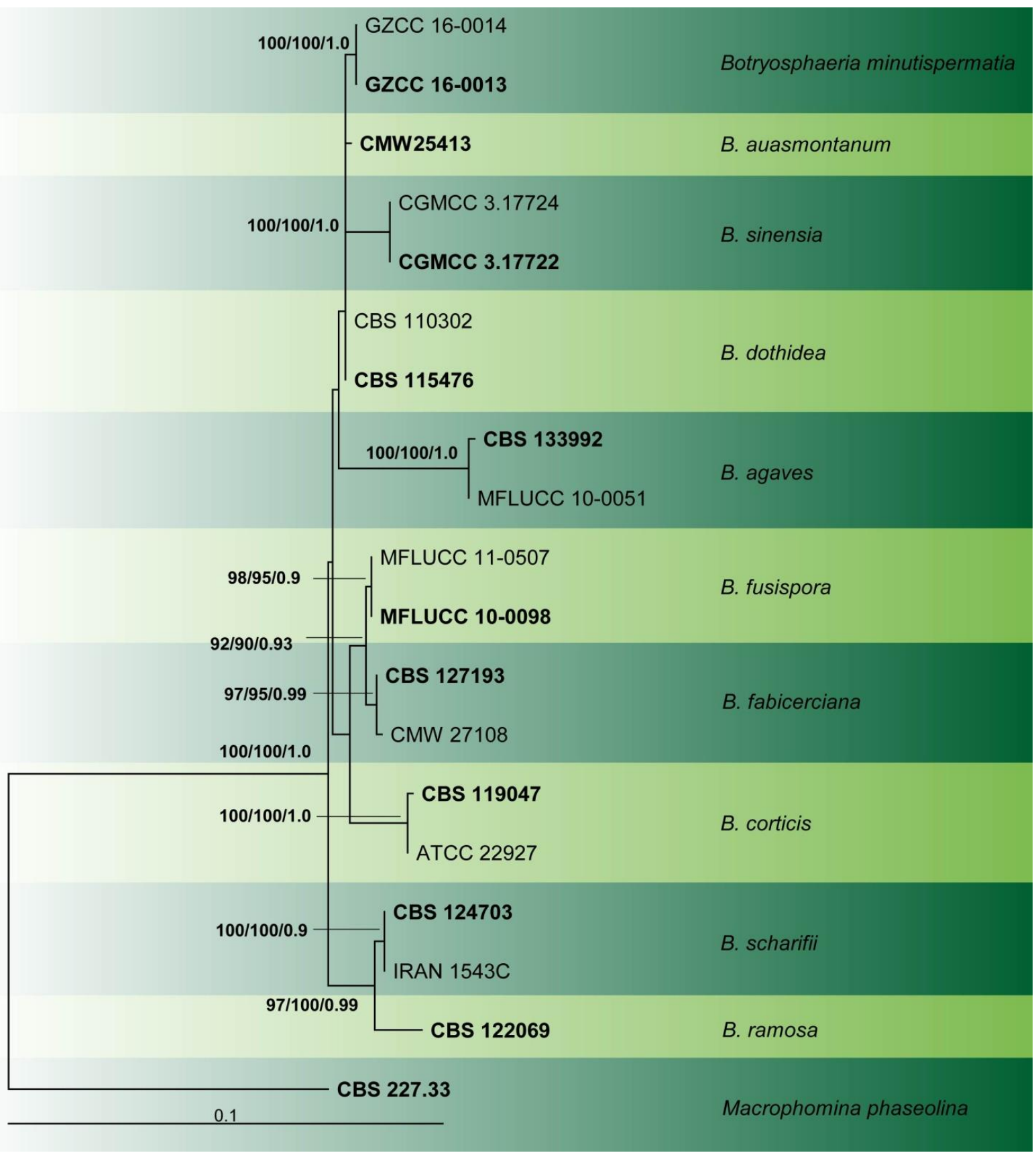

Fig. 2 - Phylogram generated from Maximum Likelihood analysis based on combined ITS and tefl- $\alpha$ dataset of Botryosphaeria. Bootstrap support values for maximum likelihood (ML), maximum parsimony (MP) greater than $75 \%$ and Bayesian posterior probabilities greater than 0.90 are indicated near the nodes. The ex-type strains are in bold and the tree is rooted with Macrophomina phaseolina (CBS 227.33).

4.1. Botryosphaeria agaves (Henn.) E.J. Butler, Annls mycol. 9: 415 (1911), MycoBank MB119799.

See Liu et al. (2012) and Phillips et al. (2013) for illustrations and descriptions of sexual morph. Asexual morph not reported.

Type - Tanzania, Zanzibar, on leaves of Agave sisalana, Zimmerman, holotype presumably lost (not in B). Thailand, Chiang Rai Province, Doi Tung, on living and dead leaves of Agave sp. (Asparagaceae), 16 Jun. 2010, R. Phookamsak (neotype designated in Phillips et al. (2013) MFLU 11-0161, culture ex-neotype MFLUCC 11-0125 = CBS 133992).

Host - Agaves sp. (Liu et al. 2012).

Distribution - Thailand (Liu et al. 2012). 
4.2. Botryosphaeria auasmontanum F.J.J. Van der Walt, Slippers \& G.J. Marais, Persoonia 33: 162 (2014), MycoBank MB518721.

Sexual morph not reported. See Slippers et al. (2014) for illustrations and descriptions of asexual morph.

Type - Namibia, Windhoek, Acacia mellifera (Fabaceae), Feb. 2006, F.J.J. van der Walt \& J. Roux (holotype PREM 59632, culture ex-type CBS 121769).

Host - Acacia mellifera (Slippers et al. 2014).

Distribution - Namibia (Slippers et al. 2014).

4.3. Botryosphaeria corticis (Demaree \& Wilcox) Arx \& E. Müll., Beitr. Kryptfl. Schweiz 11: 42 (1954), MycoBank MB293807.

Sexual morph and asexual morph have been reported. See Phillips et al. (2013) for illustrations and descriptions.

Type - USA, North Carolina, Atkinson, Vaccinium corymbosum (Ericaceae), 14 Feb. 1940, J.B. Demaree (holotype BPI 598729), New Jersey, Hammonton, on cankered stems of V. corymbosum, May 2005, P.V. Oudemans (epitype CBS H-19706, cultures ex-epitype CBS 119047, CBS 119048).

Hosts - Vaccinium species including V. ashei, V. corymbosum, V. tenellum and V. virgatum (Phillips et al. 2006, Wright \& Harmon 2010).

Distribution - USA (Florida, Georgia, Maryland, Mississippi, New Jersey, North Carolina) (Phillips et al. 2006, Wright \& Harmon 2010).

4.4. Botryosphaeria dothidea (Moug. : Fr.) Ces. \& De Not., Comm. Soc. crittog. Ital. 1: 212 (1863), MycoBank MB183247.

Sexual morph and asexual morph reported. See Phillips et al. (2013) for illustrations and descriptions.

Type - France, Rosa sp., 1823, Fries ex Mougeot. Herbarium S (neotype of Sphaeria dothidea designated by Slippers et al. 2004a). Switzerland, Ticino, Crocifisso, Prunus sp., Oct. 2000, B. Slippers (epitype PREM 57372 designated by Slippers et al. (2004a), culture ex-epitype CBS 115476). Italy, on branches of Aesculus sp., P.A. Saccardo, PAD, (neotype of asexual morph designated by Crous \& Palm 1999).

Hosts - According to USDA fungal database, this species has been reported in 171 host genera belonging to 75 families. However, the validity of many of these records is questionable since they were made prior to the re-evaluation of $B$. dothidea by Slippers et al. (2004a) who established a modern and reliable concept for this species. Studies subsequent to Phillips et al. (2013) confirm the following hosts: Acer platanoides in China (Wang et al. 2015), Artemisia sp. in China (Cosoveanu et al. 2016), Camellia sinensis in China (Jayawardena et al. 2016), Cupressus sempervirens in Iran (Mohammadi et al. 2014), Juglans regia in California, China (Chen et al. 2014a, Li et al. 2016b), Kiwifruit in China (Zhou et al. 2015), Malus domestica in Turkey, Serbia (Vasic et al. 2013, Turkolmez et al. 2016), Mangifera indica in Brazil (Marques et al. 2013b, Rabari et al. 2016), ornamental trees in Western Balkans (Zlatkovic et al. 2016), Phoenix dactylifera in Iran (Mohammadi 2014), Pistacia vera in California, Iran (Chen et al. 2014b, Mohammadi et al. 2014), Prunus serrulata in China (Yan et al. 2016), Psidium guajava in Brazil (Nogueira Junior et al. 2016), Pyrus sp. in China (Zhai et al. 2014), Tobacco in China (Bian et al. 2015), Vaccinium corymbosum in China (Xu et al. 2015b), Vitis vinifera in Algeria, China, Italy, Tunisia, Turkey (Yan et al. 2013, Ammad et al. 2014, Chebil et al. 2014, Akgul et al. 2015, Carlucci et al. 2015).

Distribution - Worldwide. 
4.5. Botryosphaeria fabicerciana (S.F. Chen, Pavlic, M.J. Wingf. \& X.D. Zhou) A.J.L. Phillips \& A. Alves, Stud. Mycol. 76: 77 (2013), MycoBank MB805457.

Sexual morph not reported. See Chen et al. (2011), Phillips et al. (2013) for illustrations and descriptions of asexual morph.

Type - China, Fujian Province, from senescing twigs of an unknown Eucalyptus sp. (Myrtaceae), Aug. 2007, M.J. Wingfield (holotype PREM 60449, culture ex-type CBS 127193).

Hosts - Eucalyptus sp. (Chen et al. 2011), Mangifera indica (Nogueira Junior et al. 2016, Marques et al. 2013b).

Distribution - China (Chen et al. 2011), Brazil (Marques et al. 2013b, Nogueira Junior et al. 2016).

4.6. Botryosphaeria fusispora Boonmee, J.K. Liu \& K.D. Hyde, Fungal Diversity 57: 171 (2012), MycoBank MB 801319.

Sexual morph and asexual morph reported. See Liu et al. (2012) for illustrations and descriptions.

Type - Thailand, Chiang Rai, Doi Tung, on dried bark of Entada sp. (Fabaceae), 10 Jun. 2009, S. Boonmee (holotype MFLU 10-0028, culture ex-type MFLUCC 10-0098).

Hosts - Caryota sp., Entada sp. (Liu et al. 2012).

Distribution - Thailand (Liu et al. 2012).

4.7. Botryosphaeria minutispermatia Ariyawansa, K.D. Hyde \& Z.Y. Liu, Phytotaxa 275: 40 (2016), Facesoffunginumber FoF02393.

Sexual morph and asexual morph reported. See Ariyawansa et al. (2016) for illustrations and descriptions.

Type - China, Guizhou Province, Guiyang City, Huaxi District, Guizhou Academy of Agricultural Sciences garden, on dead wood, 14 December 2015, HA Ariyawansa (holotype GZAAS 16-0009, culture ex-type GZCC 16-0013).

Host - Unknown host (Ariyawansa et al. 2016).

Distribution - China (Ariyawansa et al. 2016).

4.8. Botryosphaeria ramosa (Pavlic, T.I. Burgess \& M.J. Wingf.) A.J.L. Phillips \& A. Alves, Stud. Mycol. 76: 77 (2013), MycoBank MB805458.

Sexual morph not reported. See Phillips et al. (2013) for illustrations and descriptions.

Type - Australia, Western Australia, Bell Gorge, on Eucalyptus camaldulensis (Myrtaceae), Jul. 2006, T.I. Burgess (holotype PREM 59846, culture ex-type CBS 122069).

Host - Eucalyptus camaldulensis (Pavlic et al. 2008).

Distribution - Australia (Pavlic et al. 2008).

4.9. Botryosphaeria scharifii Abdoll., Zare \& A.J.L. Phillips, Mycologia 105: 213 (2013), MycoBank MB564800

Sexual morph not reported. See Abdollahzadeh et al. (2013) for illustrations and descriptions of asexual morph.

Type - Iran, Tehran, on fruits of Mangifera indica (Anacardiaceae) imported from Pakistan, Aug. 2006, J. Abdollahzadeh (holotype IRAN 14275F, culture ex-type CBS 124703).

Host - Mangifera indica (Abdollahzadeh et al. 2013).

Distribution - Iran, Pakistan (Abdollahzadeh et al. 2013).

4.10. Botryosphaeria sinensia Y.P. Zhou, Y. Zhang ter. Phytotaxa 245: 043 (2016), MycoBank MB813802. 
Sexual morph and asexual morph have been reported. See Zhou et al. (2016) for illustrations and descriptions.

Type - China, Henan Province, Puyang, Qingfeng county, on twigs of Populus sp. (Salicaceae), 11 Nov. 2014 (holotype HMAS 246714!, culture ex-type CGMCC 3.17722).

Hosts - Juglans regia, Morus sp., Populus sp. (Zhou et al. 2016).

Distribution - China (Zhou et al. 2016).

5. Cophinforma Doilom, J.K. Liu \& K.D. Hyde, Fungal Diversity 57: 174 (2012), MycoBank MB801315.

This genus comprises two species. Sexual morph and asexual morph have been reported within the genus.

Type species - Cophinforma atrovirens (Mehl \& Slippers) A. Alves \& A.J.L. Phillips 2013.

5.1. Cophinforma atrovirens (Mehl \& Slippers) A. Alves \& A.J.L. Phillips, Stud. Mycol. 76: 80 (2013), MycoBank MB805459.

Although Phillips et al. (2013) stated that the sexual morph has not been reported, Liu et al. (2012) described and illustrated the sexual morph of $C$. eucalypti, which Phillips et al. (2013) regarded as a synonym of $C$. atrovirens. See Phillips et al. (2013) for illustrations and descriptions of asexual morph.

Type - South Africa, Mpumalanga Province, Mawewe Nature Reserve, from an asymptomatic branch of Pterocarpus angolensis (Fabaceae), Dec. 2005, J.W.M. Mehl \& J. Roux (holotype PREM 60341, culture ex-type CBS 124934, paratype PREM 60342, culture ex-papartype CBS 124935).

Hosts - Eucalyptus sp. (Liu et al. 2012), Eucalyptus urophylla (Xu et al. 2015a), Pterocarpus angolensis (Mehl et al. 2011).

Distribution - South Africa (Mehl et al. 2011), Thailand (Liu et al. 2012), Venezuela (Xu et al. 2015a).

5.2. Cophinforma mamane (D.E. Gardner) A.J.L. Phillips \& A. Alves, Stud. Mycol. 76: 80 (2013), MycoBank MB805460.

Sexual morph and asexual morph reported. See Gardner (1997) for illustrations and Phillips et al. 2013 for descriptions of asexual morph.

Type - USA, Hawaii, Hawaii Island, Hawaii Volcanoes National Park, Kipuka Ki, on bark of a swollen branch of Sophora chrysophylla (Fabaceae), 1 May 1996, D.E. Gardner (holotype BISH 644614, no cultures extant; isotype BISH 737731, no cultures extant; paratypes BPI 737732, BPI 737733, no cultures extant).

Hosts - Sophora chrysophylla (Gardner 1997), Mangifera indica (Marques et al. 2013b), Vitis vinifera (Correia et al. 2013).

Distribution - Brazil (Correia et al. 2013, Marques et al. 2013b), USA (Hawaii) (Gardner 1997).

6. Diplodia Fr., in Montagne, Annls Sci. Nat., Bot., sér. 2, 1: 302 (1834), MycoBank MB8047.

This genus comprises 26 species (Fig. 3). Sexual and asexual morphs have been reported.

Type species - Diplodia mutila (Fr. : Fr.) Fr. 1849.

6.1. Diplodia africana Damm \& Crous, Mycologia 99: 671 (2008), MycoBank MB501323.

Sexual morph not reported. See Damm et al. (2007), Phillips et al. (2013) for illustrations and descriptions of asexual morph. 
Type - South Africa, Western Cape Province, Paarl, from wood section close to pruning wound of Prunus persica (Rosaceae), 10 Jun. 2004, U. Damm (holotype CBS H-19843, culture ex-type CBS 120835, additional culture STE-U 6289).

Hosts - Juniperus phoenicea (Alves et al. 2014), Prunus persica (Damm et al. 2007).

Distribution - Italy (Alves et al. 2014), South Africa (Damm et al. 2007).

6.2. Diplodia agrifolia S.C. Lynch \& Eskalen, Mycologia 105: 135 (2012), MycoBank MB800443.

Sexual morph not reported. See Lynch et al. (2013), Phillips et al. 2013 for illustrations and descriptions of asexual morph.

Type - USA, California, San Diego County, Mataguay Scout Camp, on cankered branch of Quercus agrifolia (Fagaceae), 23 Feb. 2010, S.C. Lynch \& A. Eskalen (holotype BPI 884095, culture ex-type CBS 132777 = ATCC MYA-4895 = UCROK 732).

Hosts - Quercus agrifolia, Q. kelloggii (Lynch et al. 2013).

Distribution - USA (Lynch et al. 2013).

6.3. Diplodia alatafructa Mehl \& Slippers, Mycologia 103: 542 (2011), MycoBank MB513498.

Sexual morph not reported. See Mehl et al. (2011), Phillips et al. (2013) for illustrations and descriptions of asexual morph.

Type - South Africa, Mpumalanga Province, Sudwala Caves area, from a stem wound on Pterocarpus Angolensis (Fabaceae), Dec. 2005, J.W.M. Mehl \& J. Roux (holotype PREM 60337, culture ex-type CBS 124931).

Host - Pterocarpus angolensis (Mehl et al. 2011).

Distribution - South Africa (Mehl et al. 2011).

6.4. Diplodia allocellula Jami, Gryzenh., Slippers \& M.J. Wingf., Cryptog. Mycol. 33: 256 (2012), MycoBank MB564140

Sexual morph not reported. See Jami et al. (2012), Phillips et al. (2013) for illustrations and descriptions of asexual morph.

Type - South Africa, Gauteng Province, Pretoria, from branch of Acacia karroo (Fabaceae) Nov. 2009, M. Gryzenhout \& F. Jami (holotype PREM 60701, culture ex-type CBS 130408).

Host - Acacia karroo (Jami et al. 2012).

Distribution - South Africa (Jami et al. 2012).

6.5. Diplodia bulgarica A.J.L. Phillips, J. Lopes \& Bobev, Persoonia 29: 33 (2012), MycoBank MB19632.

Sexual morph not reported. See Phillips et al. $(2012,2013)$ for illustrations and descriptions of asexual morph.

Type - Bulgaria, Plovdiv, on dead twigs of Malus sylvestris (Rosaceae), 2005, S.G. Bobev (holotype CBS H-20189, culture ex-type CBS 124254).

Host - Malus sp. (Phillips et al. 2012).

Distribution - Bulgaria, Iran (Phillips et al. 2012).

6.6. Diplodia corticola A.J.L. Phillips, A. Alves \& J. Luque, Mycologia 96: 603 (2004), MycoBank MB488568.

Sexual morph and asexual morph reported. See Phillips et al. 2013 for illustrations and descriptions.

Type - Portugal, Beira Littoral, Requeixo near Aveiro, on dead branches of Quercus suber (Fagaceae), Feb. 2002, A. Alves (holotype LISE 94839, culture ex-type CBS 112549). 


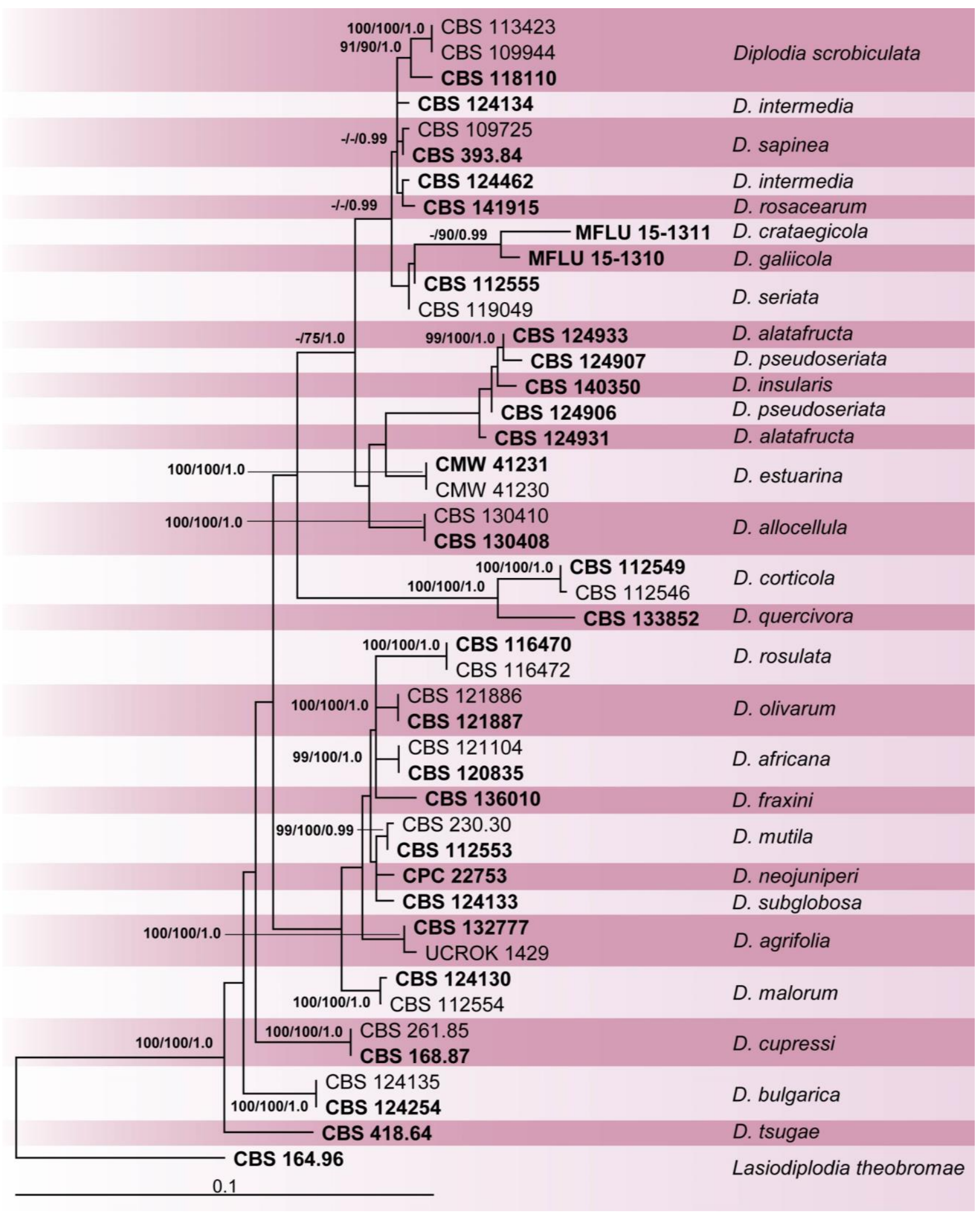

Fig. 3 - Phylogram generated from Maximum Likelihood analysis based on combined ITS and tefl- $\alpha$ dataset of Diplodia. Bootstrap support values for maximum likelihood (ML), maximum parsimony (MP) greater than $75 \%$ and Bayesian posterior probabilities above 0.90 are indicated near the nodes. The ex-type strains are in bold and the tree is rooted with Lasiodiplodia theobromae (CBS 164.96).

Hosts - Quercus sp. (Alves et al. 2004, Linaldeddu et al. 2014, Acimovic et al. 2016), Vitis vinifera (Úrbez-Torres et al. 2010, Carlucci et al. 2015).

Distribution - Italy (Carlucci et al. 2015, Linaldeddu et al. 2014), Portugal, Spain, Tunisia, USA (Alves et al. 2004, 2014), California (Úrbez-Torres et al. 2010), Maine (Acimovic et al. 2016). 
6.7. Diplodia crataegicola Dissanayake, Camporesi \& K.D. Hyde, Fungal Diversity 75: 51 (2015), Facesoffunginumber FoF00885.

Sexual morph not reported. See Ariyawansa et al. (2015) for illustrations and descriptions of asexual morph.

Type - Italy, Province of Forlì-Cesena [FC], Passo del Barbotto-Mercato Saraceno, on dead branch of Crataegus sp. (Rosaceae), 3 November 2012, E. Camporesi (holotype MFLU 15-1311, cultures ex-type MFLUCC 15-0648, KUMCC15-0075, GZCC 15-0002.

Host - Crataegus sp. (Ariyawansa et al. 2015).

Distribution - Italy (Ariyawansa et al. 2015).

6.8. Diplodia cupressi A.J.L. Phillips \& A. Alves, Fungal Diversity 23: 9 (2006), MycoBank MB510136.

Sexual morph not reported. See Phillips et al. (2013) for illustrations and descriptions of asexual morph.

Type - Israel, Bet Dagan, dried culture from cankered stems of Cupressus sempervirens (Cupressaceae), 1986, Z. Solel (holotype IMI 303475, culture ex-type CBS 168.87).

Hosts - Cupressus and Juniperus sp. (Alves et al. 2006, 2014, Solel et al. 1987).

Distribution - Cyprus, Greece, Israel, Italy, Morocco, South Africa, Tunisia, USA (de Wet et al. 2009, Alves et al. 2006, 2014, Solel et al. 1987).

6.9. Diplodia estuarina J.A Osorio, Jol. Roux \& Z.W. de Beer, Fungal Biology (available online, http://dx.doi.org/10.1016/j.funbio.2016.09.004, 2016), MycoBank MB812009.

Sexual morph not reported. See Osorio et al. (2016) for illustrations and description of asexual morph.

Type - South Africa, Kwazulu Province, St. Lucia and Richards Bay, from asymptomatic branches of Avicennia marina (Acanthaceae), J.A. Osorio \& Jol. Roux (holotype PREM 61247, culture extype CBS 139666).

Hosts - A. marina and R. mucronata (Osorio et al. 2016).

Distribution - South Africa (Osorio et al. 2016).

6.10. Diplodia fraxinii (Fr. : Fr.) Fr., Summa Veg. Scand. 2: 417. 1849. MycoBank MB247549.

Sexual morph not reported. See Alves et al. (2014) for illustrations and descriptions of asexual morph.

Type - Portugal, Monte da Caparica, on dead twigs of Fraxinus angustifolia (Oleaceae), 14 March 2013, Antonio Deidda (neotype LISE 96134, MBT176183, culture ex-neotype CBS 136010). Cascais, on dead twigs of $F$. angustifolia, 13 April 2013, Antonio Deidda (designated as morphotype A, culture CBS 136012 = CAD010).

Host - Fraxinus sp. (Alves et al. 2014).

Distribution - Italy, Netherlands, Portugal (Alves et al. 2014).

6.11. Diplodia galiicola Dissanayake, Camporesi \& K.D. Hyde, Fungal Diversity 75: 54 (2015), Facesoffunginumber FoF00884.

Sexual morph not reported. See Ariyawansa et al. (2015) for illustrations and descriptions of asexual morph. Linaldeddu et al. (2016c) consider this species a synonym of D. seriata.

Type - Italy, Province of Forlì-Cesena [FC], Strada San Zeno, Galeata, on dead stem of Galium sp. (Rubiaceae), 30 October 2013, E. Camporesi, IT 1495 (holotype MFLU 15-1310, culture ex-type MFLUCC 15-0647). 
Host - Galium sp. (Ariyawansa et al. 2015).

Distribution - Italy (Ariyawansa et al. 2015).

6.12. Diplodia insularis Linaldeddu, A. Alves \& A.J.L. Phillips, Mycosphere 7: 968 (2016), MycoBank MB818231, Facesoffunginumber FoF 02607.

Sexual morph not reported. See Linaldeddu et al. (2016c this volume) for illustrations and descriptions of the asexual morph.

Type - Italy, Santa Maria Island, isolated from a branch canker of Pistacia lentiscus (Anacardiaceae), 7 November 2013, Benedetto T. Linaldeddu, (holotype LISE 96309, culture extype CBS 140350).

Hosts - Eriobotrya japonica, Fraxinus angustifolia, Pistacia lentiscus (Linaldeddu et al. 2016c this volume).

Distribution - Caprera, Santa Maria, Sardinia (Italy) and Castellón (Spain) (Linaldeddu et al. 2016c this volume).

6.13. Diplodia intermedia A.J.L. Phillips, J. Lopes \& Bobev, Persoonia 29: 33 (2012), MycoBank MB19633.

Sexual morph and asexual morph reported. See Phillips et al. (2013) for illustrations and descriptions.

Type - Portugal, Setúbal, Monte da Caparica, dead twigs of Malus sylvestris (Rosaceae), Mar. 2006, A.J.L. Phillips (holotype CBS H-20190, culture ex-type CBS 124462).

Hosts - Cydonia, Malus (Phillips et al. 2012).

Distribution - Portugal (Phillips et al. 2012).

6.14. Diplodia malorum Fuckel, Jb. nassau. Ver. Naturk. 23-24: 395 (1870), MycoBank MB246351

Sexual morph not reported. See Phillips et al. (2013) for illustrations and descriptions of asexual morph.

Type - Germany, Rhineland, on Malus sp. (Rosaceae), 1870, J. Fuckel, Fuckel, Fungi rhenani No 1706 (holotype in G, isotypes K and M). Portugal, Setúbal, Monte da Caparica, Malus sylvestris, Feb. 2006, A.J.L. Phillips (epitype CBS H-201888, culture ex-epitype CBS 124130).

Hosts - Malus sp. (Phillips et al. 2012), Populus alba (Alves et al. 2014).

Distribution - Germany, Portugal (Phillips et al. 2012), Italy (Alves et al. 2014).

6.15. Diplodia mutila (Fr. : Fr.) Fr., Summa Veg. Scand. 2: 417 (1849), MycoBank MB201741.

Sexual morph and asexual morph reported. See Phillips et al. (2013), Alves et al. (2014) for illustrations and descriptions.

Type - France, Ardenne, Sedan, on bark of Populus nigra (Salicaceae), date unknown, Montagne (isotype K 99664). PORTUGAL, Beira Litoral, Aveiro, Populus alba, 2012, A. Alves, (epitype LISE 96136, culture ex-epitype CBS 136014).

Hosts - Recent studies subsequent to Phillips et al. (2013) confirm the following hosts: Chamaecyparis lawsoniana, Fraxinus excelsior, Fraxinus ornus, Ilex sp., Persea americana, Phoenix dactylifera, Populus alba, Prunus salicina, Taxus baccata, Vitis vinifera (Alves et al. 2014). Aesculus hippocastanum (Zlatkovic et al. 2016), Cupressus arizonica (Zlatkovic et al. 2016), Malus domestica (Úrbez-Torres et al. 2016), P. halepensis (Zlatkovic et al. 2016), Phoenix dactylifera (Mohammadi et al. 2014), Vitis vinifera (Carlucci et al. 2015). 
Distribution - British Columbia (Úrbez-Torres et al. 2016), England, Italy, Portugal, South Africa, USA (Alves et al. 2014), Italy (Carlucci et al. 2015), Iran (Mohammadi et al. 2014), Montenegro, Serbia (Zlatkovic et al. 2016).

6.16. Diplodia neojuniperi T. Trakunyingcharoen, L. Lombard \& Crous, Persoonia 34: 92 (2014), MycoBank MB810168.

Sexual morph not reported. See Trakunyingcharoen et al. (2015b) for illustrations and descriptions of asexual morph.

Type - Thailand, Chiang Mai province, on leaf of Juniperus chinensis (Cupressaceae), Feb. 2012, T. Trakunyingcharoen (holotype CBS H-21932, culture ex-type CBS 138652).

Host - Juniperus chinensis (Trakunyingcharoen et al. 2015b).

Distribution - Thailand (Trakunyingcharoen et al. 2015b).

6.17. Diplodia olivarum A.J.L. Phillips, Frisullo \& Lazzizera, Fungal Diversity 31: 67 (2008), MycoBank MB511402.

Sexual morph not reported. See Phillips et al. (2013) for illustrations and descriptions of asexual morph.

Type - Italy, Puglia, Lecce, Scorrano, Basco Belvedere, on rotting drupes of Olea europaea (Oleaceae), Dec. 2004, S. Frisullo (holotype CBS H-19914, culture ex-type CBS 121887).

Hosts - Ceratonia siliqua (Alves et al. 2014), Olea europaea (Lazzizera et al. 2008), Quercus coccifera (Alves et al. 2014).

Distribution - Italy (Lazzizera et al. 2008, Alves et al. 2014), Spain (Gramaje et al. 2012), Tunisia (Alves et al. 2014).

6.18. Diplodia pseudoseriata C.A. Pérez, Blanchette, Slippers \& M.J. Wingf., Fungal Diversity 41: 63 (2009), MycoBank MB513545.

Sexual morph not reported. See Phillips et al. (2013) for illustrations and descriptions of asexual morph.

Type - Uruguay, Paysandu, Guaviyu, isolated from asymptomatic twig of Blepharocalyx salicifolius (Myrtaceae), Aug. 2006, C. Pérez (holotype PREM 60264, culture ex-type CBS 124906 (ex-type).

Hosts - Acca sellowiana, Blepharocalyx salicifolius, Eugenia uniflora, Eugenia involucrata, Hexachlamis edulis, Myrceugenia euosma, Myrciaria tenella, Myrcianthes cisplatensis (Pérez et al. 2010), Fraxinus angustifolia (Alves et al. 2014).

Distribution - Italy (Alves et al. 2014), Uruguay (Pérez et al. 2010).

6.19. Diplodia quercivora Linald. \& A.J.L. Phillips, Mycologia 105: 1269 (2013), MycoBank MB801757.

Sexual morph not reported. See Phillips et al. (2013) for illustrations and descriptions of asexual morph.

Type - Tunisia, Tabarka, isolated from branch cankers of Quercus canariensis (Fagaceae), 20 Sep. 2006, B.T. Linaldeddu (holotype LISE 96110, culture ex-type CBS 133852).

Hosts - Quercus canariensis (Linaldeddu et al. 2013), Quercus virginiana (Dreaden et al. 2014).

Distribution - Tunisia (Linaldeddu et al. 2013), USA (Dreaden et al. 2014).

6.20. Diplodia rosacearum S. Giambra, A. Alves, J. Armengol \& S. Burruano, Mycosphere 7: 983 (2016), MycoBank MB818575. 
Sexual morph not reported. See Giambara et al. (2016) for illustrations and description of the asexual morph.

Type - Italy, Sicily, S. Maria di Gesù, isolated from a branch canker of Eriobotrya japonica (Rosaceae), March 2015, S. Giambra, (holotype LISE 96310, culture ex-holotype CBS 141915).

Host - Eriobotrya japonica (Giambara et al. 2016).

Distribution - Italy (Giambara et al. 2016).

6.21. Diplodia rosulata Gure, Slippers \& Stenlid, Mycol. Res. 109: 1010 (2005), MycoBank MB344348.

Sexual morph not reported. See Phillips et al. (2013) for illustrations and descriptions of asexual morph.

Type - Ethiopia, Southeastern Oromia, Gambo, Munessa-Shashamane Forest Enterprise, from seeds of Prunus africana (Rosaceae), 20 Jul. 2001, A. Gure (holotype CBS H-12357, culture extype CBS 116470).

Host - Prunus africana (Gure et al. 2005).

Distribution - Ethiopia (Gure et al. 2005).

6.22. Diplodia sapinea (Fr.) Fuckel, Jb. nassau. Ver. Naturk. 23-24: 393 (1870), MycoBank MB146913.

Sexual morph not reported. See Phillips et al. (2013) for illustrations and descriptions of asexual morph.

Type - Sweden, Suecia Smaland, Femsjo, on Pinus sp. (Pinaceae), E. Fries, Scleromyceti Sueciae Exsiccati No 126, Sphaeria sapinea Fries, lectotype: B, isotypes: G, K, E, UPS, C, BR, FH. The Netherlands, Gelderland, Schovenhorst, Putten, Pinetum, on cones of Pinus nigra, June 1984, H.A. van der Aa. (Epitype CBS H-18340, culture ex-epitype CBS 393.84).

Host - Mostly in Pinus sp. (Alves et al. 2014, Adamson et al. 2015, Zlatkovic et al. 2016).

Distribution - Worldwide.

6.23. Diplodia scrobiculata J. de Wet, Slippers \& M.J. Wingf., Mycol. Res. 107: 562 (2003), MycoBank MB372427.

Sexual morph not reported. See Phillips et al. (2013) for illustrations and descriptions of asexual morph.

Type - USA, Wisconsin, Jackson County, Pinus banksiana (Pinaceae), 1987, M.A. Palmer, (holotype PREM 57461, culture ex-type CBS 118110).

Hosts - Olea europaea, Arbutus unedo (Alves et al. 2014), Pinus banksiana, P. resinosa, and $P$. greggii (de Wet et al. 2003).

Distribution - Europe (France, Italy), Mexico and, USA (California, Minnesota, Wisconsin) (de Wet et al. 2003, Alves et al. 2014).

6.24. Diplodia seriata De Not., Micr. Ital., Dec. 4: no. 6 (1842), MycoBank MB180468.

Sexual morph and asexual morph reported. See Phillips et al. (2013), Alves et al. (2014) for illustrations and descriptions.

Type - Italy, on dead stems of Jasminium sp., 18 Aug. 1837, De Notaris (holotype HERB RO). Portugal, Montemor-o-Novo, on dead stems of Vitis vinifera (Vitaceae), 31 Jul. 1997, A.J.L. Phillips, (epitype CBS H-19809, culture ex-epitype CBS 112555).

Hosts - Plurivorous.

Distribution - Worldwide. 


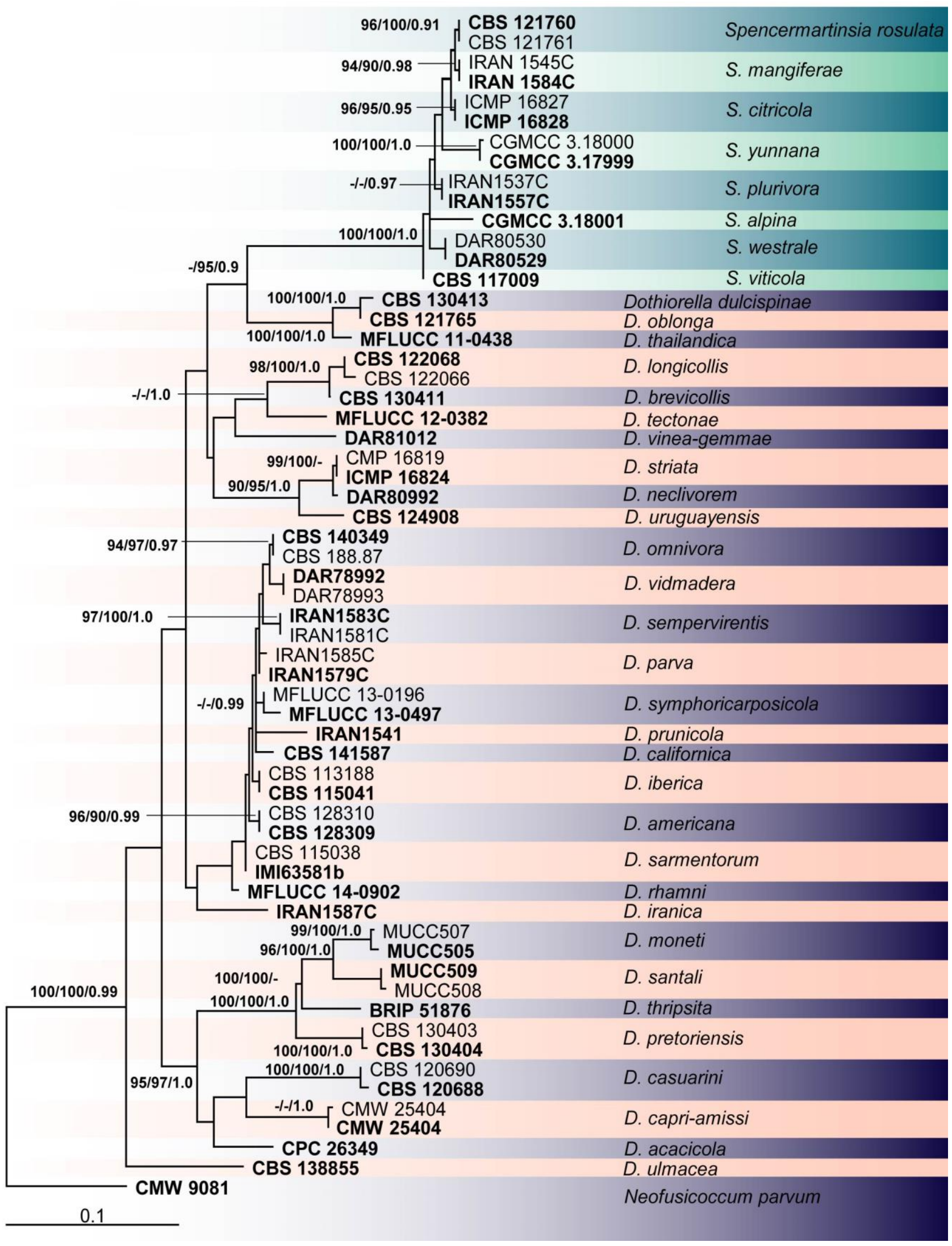

Fig. 4 - Phylogram generated from Maximum Likelihood analysis based on combined ITS and tefl- $\alpha$ dataset of Dothiorella and Spencermartinsia. Bootstrap support values for maximum likelihood (ML), maximum parsimony (MP) greater than $75 \%$ and Bayesian posterior probabilities above 0.90 are indicated near the nodes. The ex-type strains are in bold and the tree is rooted with Neofusicoccum parvum (CMW 9081). 
6.25. Diplodia subglobosa A.J.L. Phillips, Deidda \& Linald., Fungal Diversity 67: 152 (2014), MycoBank MB806049.

Sexual morph not reported. See Alves et al. (2014) for illustrations and descriptions of asexual morph.

Type - Spain, Cataluña, Lonicera nigra, (no date), J. Luque (holotype LISE 96135, culture ex-type CBS 124133). Other cultures: Spain, Cataluña, Fraxinus excelsior (Oleaceae), (no date), J. Luque (CBS 124132). Italy, Sicily, Fraxinus ornus, 2006, A. Sidoti (CBS 124131); Fraxinus excelsior, (no date) B. Slippers (CMW7776).

Hosts - Fraxinus sp., Lonicera nigra (Alves et al. 2014).

Distribution - Italy and Spain (Alves et al. 2014).

6.26. Diplodia tsugae (A. Funk) A.J.L. Phillips \& A. Alves, Persoonia 29: 35 (2012), MycoBank MB801409.

Sexual morph and asexual morph reported. See Funk (1964) for illustrations and Phillips et al. 2013, for descriptions.

Type - Canada, British Columbia, near Coola (Snootli Creek), on branches of Tsuga heterophylla (Pinaceae), 11 Sep. 1963, A. Funk (holotype DAVFP 15485. Lake Cowichan, 1 Nov. 1962, A. Funk, isotype CBS H-6790, culture ex-isotype CBS 418.64 = IMI 197143).

Host - Tsuga heterophylla (Funk 1964).

Distribution - Canada (British Columbia) (Funk 1964).

7. Dothiorella Sacc., Michelia 2: 5 (1880), MycoBank MB8098.

This genus comprises 30 species (Fig. 4). Sexual morph and the asexual morph have been reported within the genus. The number of species described in this genus has doubled since 2013 when Phillips et al (2013) included 12 species names and 16 unnamed lineages. With this increase in the number of species the phylogenetic separation between Dothiorella and Spencermartinsia has become less distinct. Nevertheless, we continue to regard these as two genera pending a detailed reevaluation.

Type species - Dothiorella pyrenophora Sacc., Michelia 2: 5. 1880.

7.1. Dothiorella acacicola Crous \& M.J. Wingf., Persoonia 36: 354 (2016), MycoBank MB817032.

Sexual morph not reported. See Crous et al. (2016) for illustrations and descriptions of asexual morph.

Type - France, La Réunion, S21²1'47.6" E55³6'48.7", on leaves of Acacia mearnsii (Fabaceae), 8 Mar. 2015, P.W. Crous \& M.J. Wingfield (holotype CBS H-22607, culture ex-type CPC $26349=$ CBS 141295).

Host - Acacia mearnsii (Crous et al. 2016).

Distribution - France (Crous et al. 2016).

7.2. Dothiorella americana Úrb.-Torr., Peduto \& Gubler, Fungal Diversity 52: 184 (2012), MycoBank MB519956.

Sexual morph not reported. See Úrbez-Torres et al. (2012) for illustrations and descriptions of asexual morph.

Type - USA, Missouri, Purdy, on diseased interspecific grape cultivar Vignoles (Ravat51), R.K. Striegler \& G.M. Leavitt (holotype UCD2252MO, culture ex-type CBS 128309).

Host - Vitis sp. (Úrbez-Torres et al. 2012). 
Distribution - USA (Missouri) (Úrbez-Torres et al. 2012).

7.3. Dothiorella brevicollis Jami, Gryzenh., Slippers \& M.J. Wingf., Cryptog. Mycol. 33: 256 (2012), MycoBank MB564142.

Sexual morph not reported. See Jami et al. (2012) for illustrations and descriptions of asexual morph.

Type - South Africa, Gauteng Province, Pretoria, from healthy wood section of Acacia karroo (Fabaceae), Nov. 2009, F. Jami (holotype PREM 60704, culture ex-type CBS 130411).

Host - Acacia karroo (Jami et al. 2012).

Distribution - South Africa (Gauteng Province) (Jami et al. 2012).

7.4. Dothiorella californica D.P. Lawr. \& Trouillas, Fungal Biology (available online, http://dx.doi.org/10.1016/j.funbio.2016.09.005, 2016), MycoBank MB817293.

Sexual morph not reported. See Lawrence et al. (2016) for illustrations and descriptions of asexual morph.

Type - USA, California: Napa County, $38.4367^{\circ} \mathrm{N}, 122.4028^{\circ} \mathrm{W}$, near Oakville 294 CA, 47 m.a.s.1., isolated from discolored wood of Umbellularia californica (Lauraceae) November 2004, F.P. Trouillas (holotype BPI 910162, culture ex-type CBS 141587).

Host - Umbellularia californica (Lawrence et al. 2016).

Distribution - California (Lawrence et al. 2016).

7.5. Dothiorella capri-amissi F.J.J. van der Walt, Slippers \& G.J. Marais, Persoonia 33: 162 (2014), MycoBank MB518723.

Sexual morph not reported. See Slippers et al. (2014) for illustrations and descriptions of asexual morph.

Type - South Africa, Prieska, from Acacia erioloba (Fabaceae), Feb. 2005, F.J.J. van der Walt \& G.J. Marais (holotype PREM 59626, culture ex-type CBS 121878).

Host - Acacia erioloba (Slippers et al. 2014).

Distribution - South Africa (Slippers et al. 2014).

7.6. Dothiorella casuarini J. de Wet, Slippers \& M.J. Wingf., Mycologia 101: 505 (2009), MycoBank MB510856.

Sexual morph not reported. See de Wet et al. (2009) for illustrations and descriptions of asexual morph.

Type - Australia, Canberra, Cotter River, on Casuarina sp. (Casuarinaceae), 2000, M.J. Wingfield (holotype PREM 59650, culture ex-type CBS 120688).

Host - Casuarina sp. (de Wet et al. 2009).

Distribution - Australia (Canberra) (de Wet et al. 2009).

7.7. Dothiorella dulcispinae Jami, Gryzenh., Slippers \& M.J. Wingf., Cryptog. Mycol. 33: 256 (2012), MycoBank MB564141.

Sexual morph not reported. See Jami et al. (2012) for illustrations and descriptions of asexual morph.

Type - South Africa, Gauteng Province, Pretoria, from die-back wood section of Acacia karroo (Fabaceae), Nov. 2009, F. Jami (holotype PREM 60706, culture ex-type CBS 130413).

Hosts - Acacia karroo (Jami et al. 2012), Acacia mellifera (Slippers et al. 2014).

Distribution - Namibia (Slippers et al. 2014), South Africa (Gauteng Province) (Jami et al. 2012). 
7.8. Dothiorella iberica A.J.L. Phillips, J. Luque \& A. Alves, Mycologia 97: 524 (2005), MycoBank MB344530.

Sexual morph and asexual morph reported. See Phillips et al. (2013) for illustrations and descriptions.

Type - Spain, Zaragoza province, Aragon, Tarazona, on dead twigs of Quercus ilex (Fagaceae), Dec. 2002, J. Luque (holotype LISE 94944, culture ex-type CBS 115041).

Hosts - Recent studies subsequent to Phillips et al. (2013) confirm the following hosts: Acer opalus (Dissanayake et al. 2016), Citrus sinensis (Adesemoye et al. 2014), Ostrya sp. (Pavlic-Zupanc et al. 2015), Pistacia sp. (Chen et al. 2014b), Prunus dulcis (Doll et al. 2015), Quercus agrifolia (Lynch et al. 2014).

Distribution - Recent studies subsequent to Phillips et al. (2013) confirm the following distribution: California (Adesemoye et al. 2014, Chen et al. 2014b, Doll et al. 2015, Lynch et al. 2014), Italy (Pavlic-Zupanc et al. 2015, Dissanayake et al. 2016).

7.9. Dothiorella iranica Abdollahz., Zare \& A.J.L. Phillips, Persoonia 32: 4 (2014), MycoBank MB803988.

Sexual morph not reported. See Abdollahzadeh et al. (2014) for illustrations and descriptions of asexual morph.

Type - Iran, Golestan Province, Gorgan (Agriculture Research Center), on twigs of Olea europea (Oleaceae), June 2007, A. Javadi (holotype IRAN 16253F, culture ex-type IRAN 1587C = CBS 124722).

Hosts - Olea europea (Abdollahzadeh et al. 2014), Paliurus sp. (Hyde et al. 2016)

Distribution - Iran (Abdollahzadeh et al. 2014), Italy (Hyde et al. 2016)

7.10. Dothiorella longicollis Pavlic, T.I. Burgess \& M.J. Wingf., Mycologia 100: 859 (2008), MycoBank MB512053.

Sexual morph not reported. See Pavlic et al. (2008) for illustrations and descriptions of asexual morph.

Type - Australia, Western Australia, Tunnel Creek National Park, on healthy branches of Lysiphyllum cunninghamii (Fabaceae), Jul. 2006, T.I. Burgess (holotype PREM 59485, culture extype CBS 122068).

Hosts - Lysiphyllum cunninghamii, Terminalia sp. (Pavlic et al. 2008)

Distribution - Australia (Pavlic et al. 2008).

7.11. Dothiorella moneti K. Taylor, Barber, G.E. Hardy \& T.I. Burgess, Mycol. Res. 113: 342 (2009), MycoBank MB511825.

Sexual morph not reported. See Taylor et al. (2009) for illustrations and descriptions of asexual morph.

Type - Australia, Western Australia, Yalgorup National Park, from healthy stem of Acacia rostellifera (Fabaceae), Jun. 2005, K.M. Taylor (holotype PERTH 07692978, culture ex-type MUCC 505 = WAC 13154).

Host - Acacia rostellifera (Taylor et al. 2009).

Distribution - Australia (Taylor et al. (2009).

7.12. Dothiorella neclivorem W.M. Pitt \& J.R. Úrbez-Torres, Australas. Pl. Path. 44: 49 (2015), MycoBank MB808287.

Sexual morph not reported. See Pitt et al. (2015) for illustrations and descriptions of asexual morph. 
Type - Australia, Pokolbin, New South Wales, berries of Chardonnay, November 2008, N. Wunderlich (holotype DAR80992, culture ex-type DAR80992).

Host - Vitis vinifera (Pitt et al. 2015).

Distribution - Australia (Pitt et al. 2015).

7.13. Dothiorella oblonga F.J.J. Van der Walt, Slippers \& G.J. Marais, Persoonia 33: 163 (2014), MycoBank MB518719.

Sexual morph not reported. See Slippers et al. (2014) for illustrations and descriptions of asexual morph.

Type - South Africa, Pretoria, Ditholo, Acacia mellifera, May 2006, F.J.J. van der Walt \& R.N. Heath (holotype PREM 59628, culture ex-type CBS 121765); Ditholo, A. mellifera (paratype PREM 59629, culture ex-paratype CBS 121766). Namibia, Rundu, A. mellifera, Feb. 2006, F.J.J. van der Walt \& J. Roux (paratype PREM 59627, culture ex-paratype CBS 121764).

Host - Acacia mellifera (Slippers et al. 2014).

Distribution - Namibia, South Africa (Slippers et al. 2014).

7.14. Dothiorella omnivora Linaldeddu, Deidda \& Scanu, Eur J Plant Pathol 146: 272 (2016), MycoBank MB812898.

Sexual morph not reported. See Linaldeddu et al. (2016a) for illustrations and descriptions of asexual morph.

Type - Italy, Aritzo, on cankered branch of Corylus avellana (Betulaceae), 09 July 2008, B.T. Linaldeddu (holotype LISE 96304, culture extype CBS 140349).

Hosts - Chamaecyparis lawsoniana, Corylus avellana, Fraxinus excelsior, Juglans regia, Ostrya carpinifolia, Thuja occidentalis, Vitis vinifera (Linaldeddu et al. 2016a).

Distribution - Australia, Bosnia and Herzegovina, France, Iran, Italy, Serbia (Linaldeddu et al. 2016a).

7.15. Dothiorella parva Abdollahz., Zare \& A.J.L. Phillips, Persoonia 32: 5 (2014), MycoBank MB803989.

Sexual morph not reported. See Abdollahzadeh et al. (2014) for illustrations and descriptions of asexual morph.

Type - Iran, Ardabil Province, Ardabil (Fandoghlo Forest Park), on twigs of Corylus avellana, July 2007, J. Abdollahzadeh \& A. Javadi (holotype IRAN 14264F, culture ex-type IRAN 1579C = CBS 124720).

Hosts - Corylus avellana (Abdollahzadeh et al. 2014), Ostrya carpinifolia (Pavlic-Zupanc et al. 2015).

Distribution - Iran (Abdollahzadeh et al. 2014), Italy (Pavlic-Zupanc et al. 2015).

7.16. Dothiorella pretoriensis (Jami, Gryzenh., Slippers \& M.J. Wingf.) Abdollahz. \& A.J.L. Phillips, Stud. Mycol. 76: 108 (2013), MycoBank MB803995.

Sexual morph not reported. See Jami et al. (2012) for illustrations and descriptions of asexual morph.

Type - South Africa, Gauteng, Pretoria, from wood of Acacia karroo (Fabaceae) with die-back symptoms, Nov. 2009, F. Jami (holotype PREM 60709, culture ex-type CBS 130404).

Host - Acacia karroo (Jami et al. 2012).

Distribution - South Africa (Jami et al. 2012). 
7.17. Dothiorella prunicola A.J.L. Phillips \& Abdollahz., Persoonia 32: 6 (2014), MycoBank MB803991.

Sexual morph not reported. See Abdollahzadeh et al. (2014) for illustrations and descriptions of asexual morph.

Type - Portugal, Algarve Province, on twigs of Prunus dulcis (Rosaceae), June 2007, E. Diogo (holotype IRAN 16252F, culture ex-type IRAN 1541C = CBS 124723).

Host - Prunus dulcis (Abdollahzadeh et al. 2014).

Distribution - Portugal (Abdollahzadeh et al. 2014).

7.18. Dothiorella rhamni Wanasinghe, Bulgakov, E.B.G. Jones \& K.D. Hyde, Fungal Diversity 78: (2016), Facesoffunginumber FoF01668.

Sexual morph not reported. See Li et al. (2016a) for illustrations and descriptions of asexual morph. Type - Russia, Rostov region, Oktyabrsky District, near natural sanctuary "Persianovskaya steppe", Khoruli hollow, ravine grove $\left(47.5006484^{\circ} \mathrm{E}, 40.1385927^{\circ} \mathrm{N}\right)$, on Rhamnus cathartica (Rhamnaceae), 26 April 2014, T.S. Bulgakov (holotype MFLU 15-3541, culture ex-type MFLUCC 14-0902).

Host - Rhamnus cathartica (Li et al. 2016a).

Distribution - Russia (Li et al. 2016a).

7.19. Dothiorella santali K. Taylor, Barber \& T.I. Burgess, Mycol. Res. 113: 345 (2009), MycoBank MB511828.

Sexual morph not reported. See Taylor et al. (2009) for illustrations and descriptions of asexual morph.

Type - Australia, Western Australia, Yalgorup National Park, from healthy stem of Santalum acuminatum (Santalaceae), Jun. 2005, K.M. Taylor (holotype PERTH 07693028, culture ex-type MUCC 509 = WAC 13155).

Host - Santalum acuminatum (Taylor et al. 2009).

Distribution - Australia (Taylor et al. 2009).

7.20. Dothiorella sarmentorum (Fr.) A.J.L. Phillips, A. Alves \& J. Luque, Mycologia 97: 522 (2005), MycoBank MB501403.

Sexual morph and asexual morph reported. See Phillips et al. (2013) for illustrations and descriptions.

Type - England, Warwickshire, on Ulmus sp. (Ulmaceae), Aug. 1956, E.A. Ellis, (holotype and culture ex-type IMI 63581b [as Otthia spiraeae]).

Hosts - Plurivorous. See Dissanayake et al. (2016) for host details (identifications are supported by molecular data of post-2005).

Distribution - Worldwide, See Dissanayake et al. (2016) for distribution of D. sarmentorum.

7.21. Dothiorella sempervirentis Abdollahz., Zare \& A.J.L. Phillips, Persoonia 32: 6 (2014), MycoBank MB803987.

Sexual morph not reported. See (Abdollahzadeh et al. 2014) for illustrations and descriptions of asexual morph.

Type - Iran, Golestan Province, Gorgan (City Park), on twigs and cones of Cupressus sempervirens (Cupressaceae), Aug. 2006, M.A. Aghajani (holotype IRAN 14265F, culture ex-type IRAN 1583C $=$ CBS 124718).

Host - Cupressus sempervirens (Abdollahzadeh et al. 2014). 
Distribution - Iran (Abdollahzadeh et al. 2014).

7.22. Dothiorella striata A.J.L. Phillips \& Abdollahz., Persoonia 32: 7 (2014), MycoBank MB803990.

Sexual morph not reported. See (Abdollahzadeh et al. 2014) for illustrations and descriptions of asexual morph.

Type - New Zealand, Northland, Kerikeri, Collins Orchard, Inlet Road, on twigs of Citrus sinensis (Rutaceae), Sept. 2006, S.R. Pennycook, P.R. Johnston \& B.C. Paulus (holotype PDD 92029, culture ex-type ICMP $16824=$ CBS 124731).

Host - Citrus sinensis (Abdollahzadeh et al. 2014).

Distribution - New Zealand (Abdollahzadeh et al. 2014).

7.23. Dothiorella symphoricarposicola W.J. Li, J.K. Liu \& K.D. Hyde, Cryptogamie Mycologie, 35: 257 (2014), Facesoffunginumber FOF000024.

Sexual morph not reported. See (Li et al. 2014) for illustrations and descriptions of asexual morph.

Type - Italy, Forlì-Cesena [FC], Berleta-Santa Sofia, on dead bark of Symphoricarpos sp. (Caprifoliaceae), 18 May 2013, Erio Camporesi, IT-1075 (holotype MFLU 14-0217, cultures extype MFLUCC 13-0497 and MFLUCC 13-0498); Spinello-Santa Sofia, on dead bark of Symphoricarpos sp., 28 June 2013, Erio Camporesi IT-1075A (paratype MFLU 14-0297.

Hosts - Laburnum alpinum, Laurus nobilis, Sambucus nigra, Symphoricarpos sp. (Li et al. 2014, Dissanayake et al. 2016).

Distribution - Italy (Li et al. 2014, Dissanayake et al. 2016).

7.24. Dothiorella tectonae Doilom, L.A. Shuttleworth, \& K.D. Hyde, Phytotaxa 233: 001 (2015), Facesoffunginumber FoF00165.

Sexual morph not reported. See Doilom et al. (2015) for illustrations and descriptions of asexual morph.

Type - Thailand, Phayao Province, Muang District, on dead branch of Tectona grandis (Lamiaceae), 12 March 2012, M. Doilom (holotype MFLU 14-0272, culture ex-type MFLUCC 120382, MUCL 55409).

Host - Tectona grandis (Doilom et al. 2015).

Distribution - Thailand (Doilom et al. 2015).

7.25. Dothiorella thailandica Abdollahz., A.J.L. Phillips \& A. Alves, Stud. Mycol. 76: 110 (2013), MycoBank MB805461.

Sexual morph not reported. See Liu et al. (2012) for illustrations and descriptions of asexual morph.

Type - Thailand, Chiang Rai Province, Doi Pui, on dead bamboo culm (Poaceae), 1 Sep. 2011, D.Q. Dai (holotype MFLU 12-0751, culture ex-type MFLUCC 11-0438 = CBS 133991).

Host - Bamboo (Liu et al. 2012).

Distribution - Thailand (Liu et al. 2012).

7.26. Dothiorella thripsita R.G. Shivas \& D.J. Tree, Persoonia 22: 169 (2009), MycoBank MB513166.

Sexual morph not reported. See Shivas et al. (2009) for illustrations and descriptions of asexual morph.

Type - Australia, Queensland, Tallegalla, on dead stems and phyllodes of Acacia harpophylla (Fabaceae), Mar. 2008, D.J. Tree \& C.E.C. Tree (holotype BRIP 51876, culture ex-type BRIP 51876). 
Host - Acacia harpophylla (Shivas et al. 2009).

Distribution - Australia (Shivas et al. 2009).

7.27. Dothiorella ulmacea Crous \& R.K. Schumach., Sydowia 67: 100 (2015), MycoBank MB812527.

Sexual morph not reported. See Crous et al. (2015b) for illustrations and descriptions of asexual morph.

Type - Germany, on a twig of Ulmus laevis (Ulmaceae), 1 Mar. 2014, leg. R. K. Schumacher (holotype CBS H-22257, culture ex-type CBS 138855); other specimen and culture on twigs of Ulmus laevis, 9 July 2014, leg. R. K. Schumacher (CBS H-22282, culture CPC 24945 = CBS 140005).

Host - Ulmus leavis (Crous et al. 2015b).

Distribution - Germany (Crous et al. 2015b).

7.28. Dothiorella uruguayensis (C.A. Pérez, Blanchette, Slippers \& M.J. Wingf.) Abdollahz. \& A.J.L. Phillips, Stud. Mycol. 76: 110 (2013), MycoBank MB803999.

Sexual morph not reported. See Pérez et al. (2010) for illustrations and descriptions of asexual morph.

Type - Uruguay, Paysandu, Tres Bocas, endophytic on twigs of Hexachlamis edulis, Aug. 2006, C.A. Pérez (holotype PREM 60268, culture ex-type CBS 124908).

Host - Hexachlamis edulis (Pérez et al. 2010).

Distribution - Uruguay (Pérez et al. 2010).

7.29. Dothiorella vidmadera W.M. Pitt, Úrbez-Torres \& Trouillas, Fungal Diversity Res. Ser. 61: 216 (2013), MycoBank MB803533.

Sexual morph and asexual morph reported. See Pitt et al. (2013b) for illustrations and descriptions of asexual morph and Li et al. (2016a) for illustrations and descriptions of sexual morph.

Type - Australia, EdenValley, South Australia, Vitis vinifera (Vitaceae) W.M. Pitt \& A. Loschiavo, (holotype DAR78992, culture ex-type DAR78992).

Hosts - Fraxinus ornus (Li et al. 2016a), Robinia pseudoacacia (Hyde et al. 2016), Vitis vinifera (Pitt et al. 2013b).

Distribution - Australia (Pitt et al. 2013b), Italy (Li et al. 2016a), Russia (Hyde et al. 2016).

7.30. Dothiorella vinea-gemmae W.M. Pitt \& J.R. Úrbez-Torres, Australas. Pl. Path. 44: 51 (2015), MycoBank MB808288.

Sexual morph not reported. See Pitt et al. (2015) for illustrations and descriptions of asexual morph.

Type - Australia, Pokolbin, New South Wales, Vitis vinifera (Vitaceae), November 2008, N. Wunderlich (holotype, DAR81012, culture ex-type, DAR81012).

Host - Vitis vinifera (Pitt et al. 2015).

Distribution - Australia (Pitt et al. 2015).

8. Endomelanconiopsis E.I. Rojas \& Samuels, Mycologia 100: 770 (2008), MycoBank MB511837. Endomelanconiopsis comprises only two species and only the asexual morph has been reported within the genus.

Type species - Endomelanconiopsis endophytica E.I. Rojas \& Samuels 2008.

8.1. Endomelanconiopsis endophytica E.I. Rojas \& Samuels, Mycologia 100: 770 (2008), MycoBank MB511838. 
Sexual morph not reported. See Rojas et al. (2008) for illustrations and descriptions of asexual morph.

Type - Panama, Nombre de Dios, isolated from leaves of Theobroma cacao (Malvaceae), 2000, E. Rojas, L. Mejía \& Z. Maynard (holotype BPI 878370, culture ex-type CBS 120379).

Hosts - Carapa guianensis (Ferreira et al. 2015), Heisteria concinna, Theobroma cacao (Rojas et al. 2008)

Distribution - Brazil (Ferreira et al. 2015), Panama (Rojas et al. 2008).

8.2. Endomelanconiopsis microspora (Verkley \& Aa) E.I. Rojas \& Samuels, Mycologia 100: 770 (2008), MycoBank MB511839.

Sexual morph not reported. See Verkley \& van der Aa (1997) for illustrations and descriptions of asexual morph.

Type - Papua New Guinea, Central Province, $22 \mathrm{~km}$ E of Port Moresby, Varirata National Park near Varirata Lookout, soil in dry secondary forest with Casuarina and Eucalyptus, and conglomerate rock outcrops, 23 Oct. 1995, A. Aptroot, H.A. van der Aa (holotype CBS H-12183, culture ex-type CBS 353.97).

Substrate - Soil (Verkley \& van der Aa 1997).

Distribution - Papua New Guinea (Verkley \& van der Aa 1997).

9. Eutiarosporella Crous, Phytotaxa 202: 85 (2015), MycoBank MB811248

This genus was introduced for tiarosporella-like fungi with long conidiomatal necks and holoblastic conidiogenesis (Crous et al. 2015a). The genus comprises seven species. Both sexual morph and asexual morph have been reported within the genus.

Type species - Eutiarosporella tritici (B. Sutton \& Marasas) Crous 2015.

9.1. Eutiarosporella africana (Jami, Gryzenh., Slippers \& M.J. Wingf.) Crous, Phytotaxa 202: 85 (2015), MycoBank MB811249.

Sexual morph not reported. See Jami et al. (2014) for illustrations and descriptions of asexual morph.

Type - South Africa, Gauteng Province, Pretoria, from healthy wood section of Celtis africana (Cannabaceae), Nov. 2011, F. Jami \& M. Gryzenhout (holotype PREM 60866, culture ex-type CBS 133854).

Host - Celtis Africana (Jami et al. 2014).

Distribution - South Africa (Jami et al. (2014).

9.2. Eutiarosporella dactylidis (Thambug., Camporesi \& K.D. Hyde) Dissanayake, Camporesi \& K.D. Hyde, Fungal Diversity 78: (2016), Facesoffunginumber FoF00334

Sexual morph and asexual morph reported. See Thambugala et al. (2014) for illustrations and descriptions.

Type - Italy, Teodorano - Meldola (province of Forlì-Cesena [FC]), on dead leaves of Dactylis glomerata (Poaceae), 15 December 2012, Erio Camporesi (holotype MFLU 14-0580, cultures extype MFLUCC 13-0276 = ICMP 20383).

Hosts - Arrhenatherum eliatus, Dactylis glomerata (Thambugala et al. 2014), Avenella flexuosa (Li et al. 2016a)

Distribution - Italy (Thambugala et al. 2014, Li et al. 2016a).

9.3. Eutiarosporella darliae E. Thynne, M.C. McDonald, M. Evans, H. Wallwork, S. Neate \& P.S. Solomon, Australas. Pl. Path. 44: 533 (2015), MycoBank MB811116. 
Sexual morph not reported. See Thynne et al. (2015) for illustrations and descriptions of asexual morph.

Type - Australia, Queensland, isolated from Triticum aestivum (Poaceae), 2012, QLD DPI, (holotype and culture ex-type DAR 82491).

Host - Triticum aestivum (Thynne et al. 2015).

Distribution - Australia (Thynne et al. 2015).

9.4. Eutiarosporella pseudodarliae E. Thynne, M.C. McDonald, M. Evans, H. Wallwork, S. Neate \& P.S. Solomon, Australas. Pl. Path. 44: 534 (2015), MycoBank MB 811117.

Sexual morph not reported. See Thynne et al. (2015) for illustrations and descriptions of asexual morph.

Type - Australia, Queensland, isolated from Triticum aestivum (Poaceae), 2011, QLD DPI (holotype and culture ex-type DAR 82489).

Host - Triticum aestivum (Thynne et al. 2015).

Distribution - Australia (Thynne et al. 2015).

9.5. Eutiarosporella tritici (B. Sutton \& Marasas) Crous, Phytotaxa 202: 85 (2015), MycoBank MB811250.

Sexual morph not reported. See (Crous et al. 2006) for illustrations and descriptions of asexual morph.

Type - South Africa: Free State Province: Heilbron, on Triticum aestivum (Poaceae), 18 Jan. 1973, W.F.O. Marasas (holotype PREM 44966, isotype IMI 186786, culture ex-type CBS 118719).

Host - Triticum aestivum (Sutton \& Marasas 1976).

Distribution - South Africa (Sutton \& Marasas 1976).

9.6. Eutiarosporella tritici-australis E. Thynne, M.C. McDonald, M. Evans, H. Wallwork, S. Neate \& P.S. Solomon, Australas. Pl. Path. 44: 532 (2015), MycoBank MB 811115.

Sexual morph not reported. See Thynne et al. (2015) for illustrations and descriptions of asexual morph. In our families tree (Fig. 1) Eutiarosporella tritici-australis appears to be more closely related to the genus Mucoharknessia. Based on ITS sequence comparison, Thynne et al. (2015) noted that some of their isolates cluster with $M$. cortaderiae. However, the morphological description of $M$. cortaderiae is in contrast with the isolates obtained from white grain disorder of wheat (Thynne et al. 2015). On account of their morphology, Thynne et al. (2015) concluded that the white grain disorder isolates reside in Eutiarosporella and not Mucoharknessia. Subsequently, $\mathrm{Li}$ et al. (2016a) introduced a new species in Mucoharknessia; M. anthoxanthii with morphology similar to Eutiarosporella. For these reasons, we consider that the position of E. tritici-australis in Eutiarosporella is tenable and needs to be re-assessed.

Type - Australia, South Australia, isolated from Triticum aestivum (Poaceae), 2012, SARDI (holotype and culture ex-type DAR 82485).

Host - Triticum aestivum (Thynne et al. 2015).

Distribution - Australia (Thynne et al. 2015).

9.7. Eutiarosporella urbis-rosarum (Jami, Gryzenh., Slippers \& M.J. Wingf.) Crous, Phytotaxa 202: 85 (2015), MycoBank MB811251.

Sexual morph not reported. See Jami et al. (2012) for illustrations and descriptions of asexual morph.

Type - South Africa: Free State Province, Bloemfontein, healthy wood of Vachellia karroo (Fabaceae), June 2008, M. Gryzenhout (holotype PREM 60698, culture ex-type CBS 130405). 
Host - Vachellia karroo (Jami et al. 2012).

Distribution - South Africa (Jami et al. 2012).

10. Lasiodiplodia Ellis \& Everh., Bot. Gaz. 21: 92 (1896), MycoBank MB8708.

This genus comprises 31 species (Fig. 5). Both sexual asexual morph have been reported within the genus. Phillips et al. (2013) and Slippers et al. (2014) have shown that morphology is not a reliable character for species differentiation and species can be recognized only from combined ITS and tefl- $\alpha$ sequence data. Cruywagen et al. (2016) suggest that hybridization between Lasiodiplodia species is widespread and further suggest that some of the currently recognized species may in fact be hybrids, e.g. L. viticola, L. missouriana, L. laeliocattletae and L. brasiliense. Nevertheless, in the following list they are treated as distinct species until hybridization is confirmed.

Type species - Lasiodiplodia theobromae (Pat.) Griff. \& Maubl., Bull. trimest. Soc. Mycol. Fr. 25: 57. 1909.

10.1. Lasiodiplodia avicenniae J.A. Osorio, Jol. Roux \& Z.W. de Beer, Fungal Biology (available online, http://dx.doi.org/10.1016/j.funbio.2016.09.004, 2016), MycoBank MB812010.

Sexual morph not reported. See Osorio et al. (2016) for illustrations and description of asexual morph.

Type - South Africa, Kwazulu-Natal Province, Beachwood and Isipingo, from asymptomatic branches of Avicennia marina (Acanthaceae), J.A Osorio \& Jol. Roux (holotype PREM 61249, cultures ex-type CBS 139670).

Host - Avicennia marina (Osorio et al. 2016).

Distribution - South Africa (Osorio et al. 2016).

10.2. Lasiodiplodia brasiliense M.S.B. Netto, M.W. Marques \& A.J.L. Phillips, Fungal Diversity 67: 134 (2014), MycoBank MB807525.

Sexual morph not reported. See Netto et al. (2014) for illustrations and descriptions of asexual morph.

Type - Brazil, Pernambuco, Farm Dan, on Mangifera indica stems (Anacardiaceae), 2010, M.W. Marques (holotype URM 85580, ex-type CMM4015 = URM7118).

Hosts - Carica papaya, Mangifera indica (Netto et al. 2014), Cocos nucifera (Rosado et al. 2016), Tectona grandis (Doilom et al. 2015), Vitis vinifera (Correia et al. 2016a).

Distribution - Brazil (Netto et al. 2014, Correia et al. 2016a, Rosado et al. 2016), Thailand (Doilom et al. 2015).

10.3. Lasiodiplodia bruguierae J.A. Osorio, Jol. Roux \& Z.W. de Beer, Fungal Biology (available online, http://dx.doi.org/10.1016/j.funbio.2016.09.004, 2016), MycoBank MB812011.

Sexual morph not reported. See Osorio et al. (2016) for illustrations and descriptions.

Type - South Africa, Kwazulu-Natal Province, Mlalazi Nature Reserve, Mtunzini, from asymptomatic branches of Bruguiera gymnorrhiza (Rhizophoraceae), J.A Osorio \& Jol. Roux (holotype PREM 61248, culture ex-type CBS 139669).

Host - Bruguiera gymnorrhiza (Osorio et al. 2016).

Distribution - South Africa (Osorio et al. 2016).

10.4. Lasiodiplodia caatinguensis I.B.L. Coutinho, F.C.O Freire, C.S. Lima \& J.E. Cardoso, Plant Pathology (available online, Doi: 10.1111/ppa.12565, 2016), MycoBank MB815122.

Sexual morph not reported. See Coutinho et al. (2016) for illustrations and descriptions of asexual morph. 


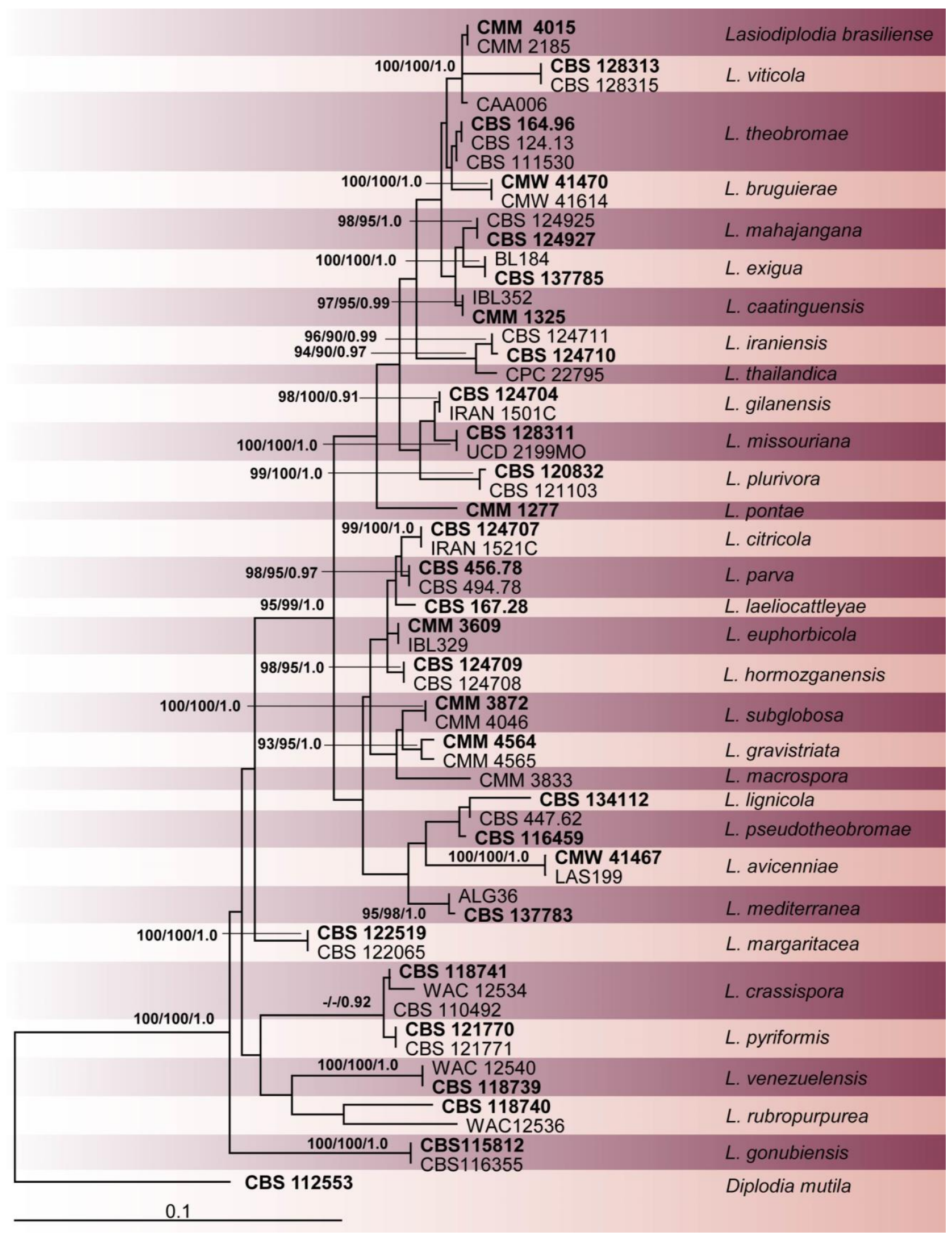

Fig. 5 - Phylogram generated from Maximum Likelihood analysis based on combined ITS and tefl- $\alpha$ dataset of Lasiodiplodia. Bootstrap support values for maximum likelihood (ML), maximum parsimony (MP) greater than $75 \%$ and Bayesian posterior probabilities above 0.90 are indicated near the nodes. The ex-type strains are in bold and the tree is rooted with Diplodia mutila (CBS 112553). 
Type - Brazil, Itarema, Ceara, trunk canker of Citrus sinensis (Rutaceae), October of 2013, I. B. L. Coutinho \& J. S. Lima (holotype VIC42498, culture ex-type CMM1325).

Host - Citrus sinensis (Coutinho et al. 2016).

Distribution - Brazil (Coutinho et al. 2016).

10.5. Lasiodiplodia citricola Abdollahz., Javadi \& A.J.L. Phillips, Persoonia 25: 4 (2010), MycoBank MB16777.

Sexual morph not reported. See Phillips et al. (2013) for illustrations and descriptions of asexual morph.

Type - Iran, Gilan Province, Chaboksar, on twigs of Citrus sp. (Rutaceae), Jun. 2007, J. Abdollahzadeh \& A. Javadi (holotype IRAN 14270F, cultures ex-type IRAN 1522C = CBS 124707).

Hosts - Citrus sp. (Abdollahzadeh et al. 2010), Juglans regia (Chen et al. 2013a, 2014a), Prunus persica (Chen et al. 2013b), Pistacia vera (Chen et al. 2014b), Vitis vinifera (Carlucci et al. 2015).

Distribution - Iran (Abdollahzadeh et al. 2010), Italy (Carlucci et al. 2015), USA (California) (Chen et al. 2013a, b, 2014a, b).

10.6. Lasiodiplodia crassispora T.I. Burgess \& Barber, Mycologia 98: 425 (2006), MycoBank MB500235.

Sexual morph not reported. See Phillips et al. (2013) for illustrations and descriptions of asexual morph.

Type - Australia, Western Australia, Kununurra, from canker on Santalum album (Santalaceae), Dec. 2003, T.I. Burgess (holotype MURU 407, cultures ex-type WAC 12533 = CMW 14691).

Hosts - Eucalyptus urophylla (Pérez et al. 2010), Mangifera indica (Marques et al. 2013a), Pterocarpus angolensis (Mehl et al. 2011), Santalum album (Burgess et al. 2006), Vitis vinifera (Úrbez-Torres et al. 2010, van Niekerk et al. 2010, Correia et al. 2016a).

Distribution - Australia (Western Australia) (Burgess et al. 2006), Brazil (Marques et al. 2013a, Correia et al. 2016a), South Africa (van Niekerk et al. 2010, Mehl et al. 2011), Uruguay (Pérez et al. 2010), USA (California) (Úrbez-Torres et al. 2010).

10.7. Lasiodiplodia euphorbiicola A.R. Machado \& O.L. Pereira, Fungal Diversity 67: 238 (2014), MycoBanK MB804872.

Synonym - Lasiodiplodia marypalmiae (as marypalme) Netto, M.W. Marques, A.J.L. Phillips \& M.P.S. Câmara, Fungal Diversity 67: 136 (2014), MycoBank MB807526.

Sexual morph not reported. See Machado et al. (2014a) for illustrations and descriptions of asexual morph. Sequences of $L$. euphorbicola were not available to Netto et al. (2014) when they described L. marypalmiae as a new species. In the phylogenetic analysis of Machado et al. (2014a) the extype isolate of L. marypalmiae nested with the ex-type of L. euphorbicola in a well-supported clade and for this reason they considered them to be synonyms.

Type - Brazil, Colatina, Espírito Santo, collar and root rot of Jatropha curcas (Euphorbiaceae), 2011, A. R. Machado \& O. L. Pereira, (holotype VIC39109, culture ex-type CMM3609).

Hosts - Carica papaya (Netto et al. 2014), Jatropha curcas (Machado et al. 2014a), Manihot esculenta (Machado et al. 2014b), Vitis vinifera (Correia et al. 2016a).

Distribution - Brazil (Machado et al. 2014a, b, Netto et al. 2014), Italy (Correia et al. 2016a).

10.8. Lasiodiplodia exigua Linaldeddu, Deidda \& A.J.L. Phillips, Fungal Diversity 71: 207 (2015), MycoBank MB808355.

Synonym - Lasiodiplodia americana S.F. Chen, G.Q. Li \& T.J. Michailides, Mycologia 107: 1 (2015), MycoBank MB810934. 
Sexual morph not reported. See Linaldeddu et al. (2015) for illustrations and descriptions of asexual morph. Rodrígez-Galvéz et al. (2016) showed that L. americana and L. exigua are phylogenetically indistinguishable and since L. exigua is the older name it takes precedence.

Type - Tunisia, Nabeul, isolated from a branch canker of Retama raetam (Fabaceae), 27 June 2012, Benedetto T. Linaldeddu (holotype LISE 96302, culture ex-type CBS 137785).

Hosts - Pistacia vera, Retama raetam (Chen et al. 2015, Linaldeddu et al. 2015).

Distribution - Tunisia (Linaldeddu et al. 2015), USA (Chen et al. 2015).

10.9. Lasiodiplodia gilanensis Abdollahz., Javadi \& A.J.L. Phillips, Persoonia 25: 5 (2010), MycoBank MB16778.

Sexual morph not reported. See Phillips et al. (2013) for illustrations and descriptions of asexual morph.

Type - Iran, Gilan Province, Rahimabad-Garmabdost, on twigs of unknown woody plant, Jun. 2007, J. Abdollahzadeh \& A. Javadi (holotype IRAN 14272F, cultures ex-type IRAN 1523C = CBS 124704).

Host - Pistacia vera (Chen et al. 2014b).

Distribution - Iran (Abdollahzadeh et al. 2010), USA (Chen et al. 2014b).

10.10. Lasiodiplodia gonubiensis Pavlic, Slippers \& M.J. Wingf., Stud. Mycol. 50: 318 (2004), MycoBank MB500079.

Sexual morph and asexual morph have been reported. See Pavlic et al. (2004) for illustrations and descriptions of the asexual morph and Trakunyingcharoen et al. (2015a) for illustrations and descriptions of the sexual morph.

Type - South Africa, Eastern Cape Province, Gonubie, isolated from Syzygium cordatum (Myrtaceae), Jul. 2002, D. Pavlic (holotype PREM 58127, culture ex-type CBS 115812).

Hosts - Phyllanthus emblica (Trakunyingcharoen et al. 2015a), Syzygium cordatum (Pavlic et al. 2004, 2007).

Distribution - South Africa (Pavlic et al. 2004, 2007), Thailand (Trakunyingcharoen et al. 2015a).

10.11. Lasiodiplodia gravistriata M.S.B. Netto \& M.P.S. Camara, Fungal Biology (available online, http://dx.doi.org/10.1016/j.funbio.2016.07.006, 2016) MycoBank MB816925.

Sexual morph not reported. See Netto et al. (2016) for illustrations and descriptions of asexual morph.

Type - Brazil, Minas Gerais, Coracao de Jesus, on Anacardium humile stems (Anacardiaceae), 2013, M. S. B. Netto (holotype URM 89942, culture ex-type CMM 4564).

Host - Anacardium humile (Netto et al. 2016).

Distribution - Brazil (Netto et al. 2016).

10.12. Lasiodiplodia hormozganensis Abdollahz., Zare \& A.J.L. Phillips, Persoonia 25: 6 (2010), MycoBank MB16779.

Sexual morph not reported. See Abdollazadeh et al. (2010) and Phillips et al. (2013) for illustrations and descriptions of asexual morph.

Type - Iran, Hormozgan Province, Rodan, on twigs of Olea sp. (Oleaceae), Jun. 2007, J. Abdollahzadeh \& A. Javadi holotype IRAN 14271F, cultures ex-type IRAN 1500C = CBS 124709).

Hosts - Bougainvillea spectabilis (Li et al. 2015), Carica papaya (Netto et al. 2014), Mangifera indica (Abdollahzadeh et al. 2010, Marques et al. 2013a, Al-sadi et al. 2013), Olea sp. 
(Abdollahzadeh et al. 2010), Phoenix dactylifera, Citrus (Al-sadi et al. 2013, 2014), Vitis vinifera (Correia et al. 2016a).

Distribution - Brazil (Marques et al. 2013a, Netto et al. 2014), China (Li et al. 2015), Iran (Hormozgan Province) (Abdollahzadeh et al. 2010), Italy (Correia et al. 2016a), Oman (Al-sadi et al. 2014), UAE (Al-sadi et al. 2013).

10.13. Lasiodiplodia iraniensis Abdollahz., Zare \& A.J.L. Phillips, Persoonia 25: 8 (2010), MycoBank MB16780.

Synonym - Lasiodiplodia jatrophicola A.R. Machado \& O.L. Pereira, Fungal Diversity 67: 239 (2014), MycoBank MB804869.

Sexual morph not reported. See Phillips et al. (2013) for illustrations and descriptions of asexual morph. Machado et al. (2014a) considered L. jatrophicola as morphologically and phylogenetically distinct from L. iraniensis. However, as pointed out by Phillips et al. (2013), morphology is not a suitable character for distinguishing species in Lasiodiplodia. Rodrígez-Galvéz et al. (2016) showed that these two species cannot be separated on the basis of ITS and tefl- $\alpha$ sequence data.

Type - Iran, Hormozgan Province, Bandar Abbas, Geno mountain, on twigs of Salvadora persica (Salvadoraceae), Mar. 2007, J. Abdollahzadeh \& A. Javadi (holotype IRAN 14268F, cultures extype IRAN $1520 \mathrm{C}=\mathrm{CBS} 124710$ ).

Hosts - Caryota mitis (Zhu et al. 2015), Citrus sp., Eucalyptus sp., Jatropha curcas (Machado et al. 2014a), Juglans sp., Mangifera indica, Salvadora persica, Terminalia catapa (Abdollahzadeh et al. 2010, Marques et al. 2013a, Sakalidis et al. 2011, Al-sadi et al. 2013, Li et al. 2015), Vitis vinifera (Correia et al. 2016a).

Distribution - Australia (Sakalidis et al. 2011), Brazil (Machado et al. 2014a, Marques et al. 2013a), China ( $\mathrm{Li}$ et al. 2015, Zhu et al. 2015), Iran (Hormozgan \& Golestan Provinces) (Abdollahzadeh et al. 2010), Italy (Correia et al. 2016a), UAE (Al-sadi et al. 2013).

10.14. Lasiodiplodia laeliocattleyae (Sibilia) A. Alves, Fungal Biology (available online, http://dx.doi.org/10.1016/j.funbio.2016.06.004, 2016), MycoBank MB815697.

Basionym - Diplodia cattleyae Sibilia, Boll. R. Staz. Patolog. Veget. Roma, N.S. 7: 433 (1927). MycoBank MB268837.

Synonym - Lasiodiplodia egyptiacae A.M. Ismail et al., Australas. Plant Path. 41: 655 (2012), MycoBank MB564516.

Sexual morph not reported. See Rodrígez-Galvéz et al. (2016) for illustrations and description of the asexual morph. Rodrígez-Galvéz et al. (2016) revealed that L. laeliocattleyae is phylogenetically indistinguishable from L. egyptiacae and therefore they correspond to the same species.

Type - Italy, from living leaves and pseudobulbs of the cultivated orchid Laeliocattleya, 1927, C. Sibilia (holotype location not known, culture ex-type of Diplodia laeliocattleyae CBS 167.28).

Hosts - Laeliocattleya, Mangifera indica (Ismail et al. 2012, Marques et al. 2013), Cocos nucifera (Rosado et al. 2016).

Distribution - Brazil (Marques et al. 2013, Rosado et al. 2016), Egypt (Ismail et al. 2012), Italy, Peru (Rodrígez-Galvéz et al. 2016).

10.15. Lasiodiplodia lignicola (Ariyaw., J.K. Liu \& K.D. Hyde) A.J.L. Phillips, A. Alves \& Abdollahz., Stud. Mycol. 76: 120 (2013), MycoBank MB805462.

Basionym - Auerswaldia lignicola Ariyawansa, J.K. Liu \& K.D. Hyde, Fungal Diversity 57: 161. 2012.

Sexual morph and asexual morph reported. See Liu et al. (2012) for illustrations and descriptions of sexual morph (as Auerswaldia lignicola) and Phillips et al. (2013) for illustrations and descriptions of asexual morph. In Phillips et al. (2013) and other phylogenetic studies, the phylogenetic position 
of L. lignicola was unclear. Cruywagen et al. (2016) re-sequenced tefl- $\alpha$ of the ex-type strain and this sequence (KU887003) was used in the analyses in the present paper, which clearly places this species in Lasiodilodia.

Type - Thailand, Chiang Rai Province, Muang District, Bandu, on dead wood, 30 Sep. 2011, A.D. Ariyawansa (holotype MFLU 12-0750, cultures ex-type MFLUCC 11-0435 = CBS 134112).

Hosts - Dead wood of unknown host (Liu et al. 2012).

Distribution - Thailand (Liu et al. 2012).

10.16. Lasiodiplodia macrospora A.R. Machado \& O.L. Pereira, Fungal Diversity 67: 240 (2014), MycoBanK MB804871.

Sexual morph not reported. See Machado et al. (2014a) for illustrations and descriptions of asexual morph.

Type - Brazil, Colatina, Espírito Santo, Collar and root rot of Jatropha curcas (Euphorbiaceae), 2011, A. R. Machado \& O. L. Pereira (holotype VIC39111, culture ex-type CMM3833).

Host - Jatropha curcas (Machado et al. 2014a).

Distribution - Brazil (Machado et al. 2014a).

10.17. Lasiodiplodia mahajangana Begoude, Jol. Roux \& Slippers, Mycol. Progr. 9: 110 (2010), MycoBank MB514012.

Sexual morph not reported. See Didier Begoude et al. (2010) for illustrations and descriptions of asexual morph.

Type - Madagascar, Mahajanga, isolated from healthy branches of Terminalia catappa (Combretaceae), Oct. 2007, J. Roux (holotype PREM 60288 holotype, culture ex-type CBS 124925).

Hosts - Euphorbia ingens (van der Linde et al. 2011), Terminalia catappa (Didier Begoude et al. 2010).

Distribution - Madagascar (Didier Begoude et al. 2010), South Africa (van der Linde et al. 2011).

10.18. Lasiodiplodia margaritacea Pavlic, T.I. Burgess \& M.J. Wingf., Mycologia 100: 860 (2008), MycoBank MB512052.

Sexual morph not reported. See Pavlic et al. (2008) for illustrations and descriptions of asexual morph.

Type - Australia, Western Australia, Tunnel Creek Gorge, on Adansonia gibbosa (Malvaceae), Jul. 2006, T.I. Burgess (holotype PREM 59844, culture ex-type CBS 122519).

Host - Adansonia gibbosa (Pavlic et al. 2008).

Distribution - Australia (Pavlic et al. 2008).

10.19. Lasiodiplodia mediterranea Linaldeddu, Deidda \& Berraf-Tebbal, Fungal Diversity 71: 207 (2015), MycoBank MB808356.

Sexual morph not reported. See Linaldeddu et al. (2015) for illustrations and descriptions of asexual morph.

Type - Italy, Bortigiadas, isolated from a branch canker of Quercus ilex (Fagaceae), June 2004, Benedetto T. Linaldeddu (holotype LISE 96303, culture ex-type CBS 137783).

Hosts - Citrus sinensis, Quercus ilex, Vitis vinifera (Linaldeddu et al. 2015, Andolfi et al. 2016).

Distribution - Algeria, Italy (Linaldeddu et al. 2015, Andolfi et al. 2016). 
10.20. Lasiodiplodia missouriana Úrb.-Torr., Peduto \& Gubler, Fungal Diversity 55: 181 (2012), MycoBank MB519954.

Sexual morph not reported. See Úrbez-Torres et al. (2012) for illustrations and descriptions of asexual morph.

Type - USA, Saint James, on Vitis vinifera $\times$ V. labrusca hybrid cv. Catawba (Vitaceae), Jun. 2006, R.K. Striegler \& G.M. Leavitt (holotype UCD2193MO, culture ex-type CBS 128311).

Host - Vitis sp. (Úrbez-Torres et al. 2012).

Distribution - USA (Úrbez-Torres et al. 2012).

10.21. Lasiodiplodia parva A.J.L. Phillips, A. Alves \& Crous, Fungal Diversity 28: 9 (2008), MycoBank MB510942.

Sexual morph not reported. See Phillips et al. (2013) for illustrations and descriptions of asexual morph.

Type - Colombia, Dep. Meta, Villavicencio, cassava field soil, 1978, O. Rangel (holotype CBS H19915, culture ex-type CBS 456.78).

Hosts - Cassava-field soil, Terminalia sp. (Begoude et al. 2011), Theobroma cacao (Alves et al. 2008), Vitis vinifera (Correia et al. 2013).

Distribution - Colombia, Sri Lanka (Alves et al. 2008), Cameroon (Begoude et al. 2011), Brazil (Correia et al. 2013).

10.22. Lasiodiplodia plurivora Damm \& Crous, Mycologia 99: 674 (2008), MycoBank MB501322.

Sexual morph not reported. See Damm et al. (2007) for illustrations and descriptions of asexual morph.

Type - South Africa, Western Cape Province, Stellenbosch, from V-shaped necrotic lesion of Prunus salicina (Rosaceae), May 2004, U. Damm (holotype CBS H-19844, culture ex-type CBS 120832).

Hosts - Prunus salicina, Vitis vinifera (Damm et al. 2007).

Distribution - South Africa (Western Cape Province) (Damm et al. 2007).

10.23. Lasiodiplodia pontae F.C.O Freire, I.B.L Coutinho, C.S. Lima \& J.E. Cardoso, Plant Pathology (available online, Doi: 10.1111/ppa.12565, 2016), MycoBank MB815121.

Sexual morph not reported. See Coutinho et al. (2016) for illustrations and descriptions of asexual morph.

Type - Brazil, Pio IX, Piaui, necrotic canker on Spondias purpurea (Anacardiaceae), October 2009, J. S. Lima \& F. C. O. Freire (holotype VIC42947, culture ex-type CMM1277).

Host - Spondias purpurea (Coutinho et al. 2016).

Distribution - Brazil (Coutinho et al. 2016).

10.24. Lasiodiplodia pseudotheobromae A.J.L. Phillips, A. Alves \& Crous, Fungal Diversity 28: 8 (2008), MycoBank MB510941.

Sexual morph reported by Tennakoon et al. (2016). Phillips et al. (2013) for illustrations and descriptions of asexual morph.

Type - Costa Rica, San Carlos, on Gmelina arborea (Lamiaceae), J. Carranza-Velazquez (holotype CBS H-19916, culture ex-type CBS 116459).

Hosts - Recent studies subsequent to Phillips et al. (2013) confirm the following hosts: Acacia mangium, Citrus aurantium, Coffea sp., Gmelina arborea, Rosa sp. (Alves et al. 2008, Castro- 
Medina et al. 2014), Bougainvillea spectabilis (Li et al. 2015), Carica papaya (Netto et al. 2014), Cocos nucifera (Rosado et al. 2016), Eucalyptus grandis (Pillay et al. 2013), Jatropha curcas (Machado et al. 2014a), Juglans regia China (Li et al. 2016b), Mangifera indica (SandovalSanchez et al. 2013, Marques et al. 2013a), Manihot esculenta Brazil (Machado et al. 2014b), Persea americana, Hevea brasiliensis (Trakunyingcharoen et al. 2015b), Schizolobium parahyba (Mehl et al. 2014), Tectona grandis (Doilom et al. 2015), Vitis vinifera (Correia et al. 2013, Correia et al. 2016a, Dissanayake et al. 2015a).

Distribution - Brazil (Marques et al. 2013a, Machado et al. 2014a, b, Correia et al. 2013, Netto et al. 2014, Rosado et al. 2016), China (Dissanayake et al. 2015a, Li et al. 2015, 2016), Costa Rica, Netherlands, Suriname, Zaire (Alves et al. 2008), Ecuador (Mehl et al. 2014), Italy (Correia et al. 2016a), Mexico (Sandoval-Sanchez et al. 2013), South Africa (Pillay et al. 2013), Thailand (Doilom et al. 2015, Trakunyingcharoen et al. 2015b), Venezuela (Castro-Medina et al. 2014).

10.25. Lasiodiplodia pyriformis F.J.J. Van der Walt, Slippers \& G.J. Marais, Persoonia 33: 163 (2014), MycoBank MB518722.

Sexual morph not reported. See Slippers et al. (2014) for illustrations and descriptions of asexual morph.

Type - Namibia, Dordabis, from Acacia mellifera (Fabaceae), Feb. 2006, F.J.J. van der Walt \& J. Roux (holotype PREM 59633, culture ex-type CBS 121770).

Host - Acacia mellifera (Slippers et al. 2014).

Distribution - Namibia (Slippers et al. 2014).

10.26. Lasiodiplodia rubropurpurea T.I. Burgess, Barber \& Pegg, Mycologia 98: 431 (2006), MycoBank MB500236.

Sexual morph not reported. See Burgess et al. 2006) for illustrations and descriptions of asexual morph.

Type - Australia, Queensland, Tully, from canker on Eucalyptus grandis (Myrtaceae), May 2003, T.I. Burgess (holotype MURU 409, culture ex-type CBS 118740).

Host - Eucalyptus grandis (Burgess et al. 2006).

Distribution - Australia (Queensland) (Burgess et al. 2006).

10.27. Lasiodiplodia subglobosa A.R. Machado \& O.L. Pereira, Fungal Diversity 67: 241 (2014), MycoBanK MB804870.

Sexual morph not reported. See Machado et al. (2014a) for illustrations and descriptions of asexual morph.

Type - Brazil, Jaíba, Minas Gerais, Collar and root rot of Jatropha curcas (Euphorbiaceae), 2011, A. R. Machado \& O. L. Pereira (holotype VIC39112, culture ex-type CMM3872).

Hosts - Carya illinoinensis (Poletto et al. 2016), Jatropha curcas (Machado et al. 2014a).

Distribution - Brazil (Machado et al. 2014a, Poletto et al. 2016).

10.28. Lasiodiplodia thailandica T. Trakunyingcharoen, L. Lombard \& Crous, Persoonia 34: 95 (2014), MycoBank MB810169.

Sexual morph not reported. See Trakunyingcharoen et al. (2015b) for illustrations and descriptions of asexual morph.

Type - Thailand, Chiang Mai province, on twigs of Mangifera indica (Anacardiaceae), May 2012, T. Trakunyingcharoen (holotype CBS-H 21933, culture ex-type CBS 138760).

Host - Mangifera indica (Trakunyingcharoen et al. 2015b).

Distribution - Thailand (Trakunyingcharoen et al. 2015b). 
10.29. Lasiodiplodia theobromae (Pat.) Griffon \& Maubl., Bull. Soc. mycol. Fr. 25: 57 (1909), MycoBank MB188476.

Sexual and asexual morph reported but the connection between the two morphs has not been proven conclusively (Phillips et al. 2013). See Phillips et al. (2013) for illustrations and descriptions.

Type - Ecuador, on Theobroma cacao, Lagerheim, holotype not found, and presumably lost. Papua New Guinea, Madang, Jais Aben, from unidentified fruit along coral reef coast, No. 1995, A. Aptroot (neotype CBS H-21411, culture ex-neotype CBS 164.96.

Hosts - Wide host range.

Distribution - Widely distributed in tropical and subtropical regions.

10.30. Lasiodiplodia venezuelensis T.I. Burgess, Barber \& Mohali, Mycologia 98: 432 (2006), MycoBank MB500237.

Sexual morph not reported. See Burgess et al. (2006) for illustrations and descriptions of asexual morph.

Type - Venezuela, Estado Portuguesa, Acarigua, from wood of living Acacia mangium (Fabaceae), Oct. 2003, S. Mohali (holotype MURU 413, culture ex-type CBS 118739).

Host - Acacia mangium (Burgess et al. 2006).

Distribution - Venezuela (Burgess et al. 2006).

10.31. Lasiodiplodia viticola Úrb.-Torr., Peduto \& Gubler, Fungal Diversity 55: 183 (2012), MycoBank MB519966.

Sexual morph not reported. See Úrbez-Torres et al. (2010) for illustrations and descriptions of asexual morph.

Type - USA, Arkansas, Altus, on interspecific hybrid grape Vignoles cv. Ravat 51R, D. Cartwright \& W. D. Gubler (holotype UCD2553AR, cultures ex-type UCD2553AR = CBS 128313).

Host - Vitis hybrids (Úrbez-Torres et al. 2010).

Distribution - USA (Arkansas and Missouri) (Úrbez-Torres et al. 2010).

11. Macrophomina Petr., Annls mycol. 21: 314 (1923), MycoBank MB8814.

This genus comprises only one species. Sexual morph not reported.

Type species - Macrophomina phaseolina (Tassi) Goid., Annali Sper. agr. N.S. 1: 457. 1947.

11.1. Macrophomina phaseolina (Tassi) Goid., Annali Sper. agr, N.S. 1: 457 (1947), MycoBank MB300023.

Sexual morph not reported. See Phillips et al. (2013) for illustrations and descriptions of asexual morph.

Holotype apparently lost. Cultures: Niger, Vigna minima, M. Ndiaye, CPC 11052, 11070. Senegal, soil, M. Ndiaye, CPC 11079, 11085, 11106, 11108. Uganda, Eucalyptus sp. (Myrtaceae), Jan. 1925, CBS 162.25; Unknown, Zea mays, Jun. 1933, S.F. Ashby, CBS 227.33.

Host - Plurivorous.

Distribution - Cosmopolitan.

12. Marasasiomyces Crous, Phytotaxa 202: 86 (2015), MycoBank MB811252.

This genus was introduced to accommodate tiarosporella-like fungi and comprises only one species. Sexual morph not reported.

Type species - Marasasiomyces karoo (B. Sutton \& Marasas) Crous 2015. 
12.1. Marasasiomyces karoo (B. Sutton \& Marasas) Crous, Phytotaxa 202: 86 (2015), MycoBank MB811253.

Sexual morph not reported. See Sutton \& Marasas (1976) and Crous et al. 2006 for illustrations and descriptions of asexual morph.

Type - South Africa. Cape Province: Colesberg, on dead stems of Eriocephalus sp. (Asteraceae), Feb. 1971, W.F.O. Marasas (holotype PREM 44967, isotype IMI 186782, culture ex-type CBS 118718).

Hosts - Eriocephalus sp., Nestlera sp., Tribulus terrestris (Sutton \& Marasas 1976).

Distribution - South Africa (Sutton \& Marasas 1976).

13. Mucoharknessia Crous, R.M. Sánchez \& Bianchin., Phytotaxa 202: 86 (2015), MycoBank MB811254.

This genus was introduced for species resembling Harknessia with conidia bearing mucoid appendages. Mucoharknessia comprises two species. Only the asexual morph has been reported within the genus.

Type species - Mucoharknessia cortaderiae Crous, R.M. Sánchez \& Bianchin 2015.

13.1. Mucoharknessia cortaderiae Crous, R.M. Sánchez \& Bianchin., Phytotaxa 202: 86 (2015), MycoBank MB811255.

Sexual morph not reported. See Crous et al. (2015a) for illustrations and descriptions of asexual morph.

Type - Argentina. Buenos Aires Province, Punta Alta, 3847’27,6”S 626'48,6”W, on leaves of Cortaderia selloana (Poaceae), 29 Mar. 2011, F.E. Anderson (holotype BBB, isotype CBS H21853, culture ex-isotype CBS 131032).

Host - Cortaderia selloana (Crous et al. 2015a).

Distribution - Argentina (Crous et al. 2015a).

13.2. Mucoharknessia anthoxanthii Dissanayake, Camporesi \& K.D. Hyde, Fungal Diversity 78 : 19 (2016), Facesoffungi number FoF01651.

Sexual morph not reported. See Li et al. (2016a) for illustrations and descriptions of asexual morph. Type - Italy, Province of Forlì-Cesena [FC], Passo delle Forche - Galeata, on dead stem of Anthoxanthum odoratum (Poaceae), 24 November 2012, Erio Camporesi (holotype MFLU 153477, culture ex-type MFLUCC 15-0904).

Host - Anthoxanthum odoratum (Li et al. 2016a).

Distribution - Italy (Li et al. 2016a).

14. Neodeightonia C. Booth, Mycol. Pap. 119: 17 (1970), MycoBank MB3450.

This genus comprises six species. Both sexual and the asexual morphs have been reported within the genus.

Type species - Neodeightonia subglobosa Booth, in Punithalingam 1970.

14.1. Neodeightonia licuriensis A. R. Machado \& O. L. Pereira, Cryptog. Mycol. 36: 121 (2015), MycoBank MB810662.

Sexual morph not reported. See Adamcik et al. (2015) for illustrations and descriptions of asexual morph.

Type - Brazil, along highway between the cities Castro Alves and Santa Terezinha, Bahia, on necrotic lesions on the rachis and leaves of Syagrus coronata (Arecaceae), 2010, O. L. Pereira, (holotype VIC42826, culture ex-type COAD1780). 
Host - Syagrus coronate (Adamcik et al. 2015).

Distribution - Brazil (Adamcik et al. 2015).

14.2. Neodeightonia palmicola J.K. Liu, Phook. \& K.D. Hyde, Sydowia 62: 268 (2010), MycoBank MB518804.

Sexual morph and asexual morph reported. See Liu et al. (2012) for illustrations and descriptions.

Type - Thailand, Chiang Rai, Muang District, Khun Korn Waterfall, on dead leaves of Arenga westerhoutii (Arecaceae), 18 Dec. 2009, Jian-Kui Liu (holotype MFLU10 0407, culture ex-type MFLUCC10 0822 = CBS 136074).

Host - Arenga westerhoutii (Liu et al. 2012).

Distribution - Thailand (Liu et al. 2012).

14.3. Neodeightonia phoenicum A.J.L. Phillips \& Crous, Persoonia 21: 43 (2008), MycoBank MB511708.

Sexual morph not reported. See Phillips et al. (2013) for illustrations and descriptions of asexual morph.

Type - Spain, Catalonia, Tarragona, Salou, on Phoenix sp. (Arecaceae), F. Garcia (holotype CBS H-20108, culture ex-type CBS 122528).

Host - Phoenix sp. (Phillips et al. 2008, Ligoxigakis et al. 2013).

Distribution - Spain, USA (California) (Phillips et al. 2008), Greece (Ligoxigakis et al. 2013).

14.4. Neodeightonia rattanica Konta \& K.D. Hyde, Mycosphere 7: 953 (2016), Facesoffunginumber FoF02237.

Sexual morph and asexual morph reported. See Konta et al. (2016b) for illustrations and descriptions.

Type - Thailand, Phang-Nga, on dead rachis of Calamus sp. (Arecaceae), 6 December 2014, S. Konta (holotype MFLU 15-1443, culture ex-type MFLUCC 15-0712).

Host - Calamus sp. (Konta et al. 2016b).

Distribution - Thailand (Konta et al. 2016b).

14.5. Neodeightonia rattanicola Konta \& K.D. Hyde, Mycosphere 7: 954 (2016), Facesoffunginumber FoF02238.

Sexual morph and asexual morph reported. See Konta et al. (2016b) for illustrations and descriptions.

Type - Thailand, Phang-Nga, on dead rachis of Calamus sp. (Arecaceae), 6 December 2014, S. Konta (holotype MFLU 15-0294, culture ex-type MFLUCC 15-0319).

Host - Calamus sp. (Konta et al. 2016b).

Distribution - Thailand (Konta et al. 2016b).

14.6. Neodeightonia subglobosa C. Booth, Mycol. Pap. 119: 19 (1970), MycoBank MB318601.

Sexual morph and asexual morph reported. See Phillips et al. (2013) for illustrations and descriptions.

Type - Sierra Leone, Njala (Kori), on dead culms of Bambusa arundinacea (Poaceae), 17 Aug. 1954, F.C. Deighton (holotype IMI 57769(f), culture ex-type CBS 448.91).

Host - Bambusa arundinacea (Punithalingam 1970).

Distribution - Sierra Leone (Punithalingam 1970). 


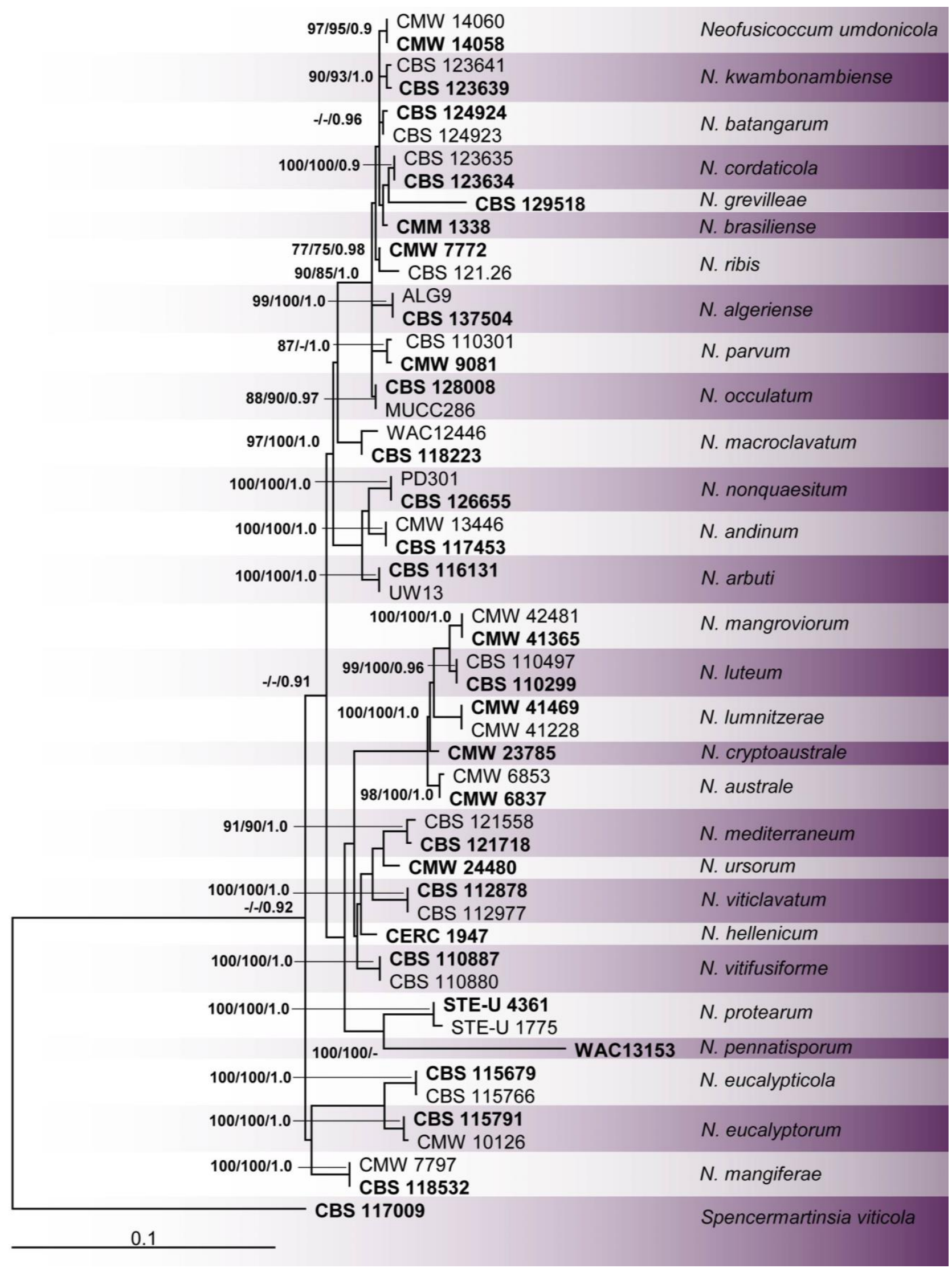

Fig. 6 - Phylogram generated from Maximum Likelihood analysis based on combined ITS and tefl- $\alpha$ dataset of Neofusicoccum. Bootstrap support values for maximum likelihood (ML), maximum parsimony (MP) greater than $75 \%$ and Bayesian posterior probabilities greater than 0.90 are indicated near the nodes. The ex-type strains are in bold and the tree is rooted with Spencermartinsia viticola (CBS 117009). 
15. Neofusicoccum Crous, Slippers \& A.J.L. Phillips, Stud. Mycol. 55: 247 (2006), MycoBank MB500870.

This genus comprises 29 species (Fig. 6). Both sexual and asexual morphs have been reported within the genus.

Type species - Neofusicoccum parvum (Pennycook \& Samuels) Crous, Slippers \& A.J.L. Phillips, Stud. Mycol. 55: 248. 2006.

15.1. Neofusicoccum algeriense Berraf-Tebbal \& A.J.L. Phillips, Fungal Diversity 53: 423 (2014), MycoBank MB808496.

Sexual morph not reported. See Berraf-Tebbal et al. (2014) for illustrations and descriptions of asexual morph. Lopes et al. (2016) consider this species as a synonym of $N$. parvum.

Type - Algeria. Ain-Benian, on trunks and branches of Vitis vinifera (Vitaceae), May 2013, A. Berraf-Tebbal (holotype LISE 9630, culture ex-type CBS 137504).

Host - Vitis vinifera (Berraf-Tebbal et al. 2014).

Distribution - Algeria (Berraf-Tebbal et al. 2014).

15.2. Neofusicoccum andinum (Mohali, Slippers \& M.J. Wingf.) Mohali, Slippers \& M.J. Wingf., Stud. Mycol. 55: 247 (2006), MycoBank MB500871.

Sexual morph not reported. See Mohali et al. (2006) for illustrations and descriptions of asexual morph.

Type - Venezuela, Mérida Morph, Merida, Mucuchies (3140 m), Cordillera of Los Andes, on branches of Eucalyptus sp. (Myrtaceae), Feb. 2003, S. Mohali (holotype PREM 58238, culture extype CBS 117453).

Host - Eucalyptus sp. (Mohali et al. 2006).

Distribution - Venezuela (Mohali et al. 2006).

15.3. Neofusicoccum arbuti (D.F. Farr \& M. Elliott) Crous, Slippers \& A.J.L. Phillips, Stud. Mycol. 55: 248 (2006), MycoBank MB500872.

Sexual morph not reported. See Phillips et al. (2013) for illustrations and descriptions of asexual morph.

Type - USA, Washington, King Co., Seattle, Magnolia Bluffs, isolated from cankers of Arbutus menziesii (Ericaceae), Oct. 2003, collected by M. Elliott, isolated by A. Rossman (holotype BPI 843970, culture ex-type CBS 116131).

Hosts - Arbutus menziesii (Pacific madrone) (Farr et al. 2005, Mcgregor et al. 2016), Vaccinium sp. (Espinoza et al. 2009).

Distribution - USA, Canada (Farr et al. 2005), Chile (Espinoza et al. 2009), North America (Mcgregor et al. 2016).

15.4. Neofusicoccum australe (Slippers, Crous \& M.J. Wingf.) Crous, Slippers \& A.J.L. Phillips, Stud. Mycol. 55: 248 (2006), MycoBank MB500873.

Sexual morph and asexual morph reported. See Phillips et al. (2013) for illustrations and descriptions.

Type - Australia, Victoria, Batemans Bay, Acacia sp. (Fabaceae), M.J. Wingfield (holotype PREM 57589, culture ex-type CMW 6837).

Hosts - Acacia karroo (Jami et al. 2015), Eucalyptus grandis, Syzygium cordatum (Pillay et al. 2013), Mangifera indica (Ismail et al. 2013), Olea europaea (Triki et al. 2015), Pistacia vera (Chen et al. 2014b), Quercus robur (Barradas et al. 2013), Vaccinium (Castillo et al. 2013), Vitis vinifera (Berraf-Tebbal et al. 2014, Pitt et al. 2013a, Valencia et al. 2015, Billones-Baaijens et al. 2015). 
Distribution - Algeria (Berraf-Tebbal et al. 2014), Australia (Pitt et al. 2013a), Chile (Valencia et al. 2015), Italy (Ismail et al. 2013, New Zealand (Billones-Baaijens et al. 2015), Portugal (Barradas et al. 2013), South Africa (Jami et al. 2015), South Africa (Pillay et al. 2013), Spain (Castillo et al. 2013), Tunisia (Triki et al. 2015), USA (Chen et al. 2014b).

15.5. Neofusicoccum batangarum Begoude, Jol. Roux \& Slippers, Stud. Mycol. 76: 137 (2013), MycoBank MB514013.

Sexual morph not reported. See Didier Begoude et al. (2010) for illustrations and descriptions of asexual morph.

Type - Cameroon, Kribi, Beach, isolated from healthy branches of Terminalia catappa (Combretaceae), Dec. 2007, D. Begoude \& J. Roux, a dry culture on pine needles (holotype PREM 60285, culture ex-type CBS 124924).

Hosts - Prickly Pear Cactus (Conforto et al. 2016), Schinus terebinthifolius (Shetty et al. 2011), Terminalia catappa (Didier Begoude et al. 2010).

Distribution - Brazil (Conforto et al. 2016), Cameroon (Didier Begoude et al. 2010), USA (Shetty et al. 2011).

15.6. Neofusicoccum brasiliense M.W. Marques, A.J.L. Phillips \& M.P.S. Câmara, sp. nov., MycoBank MB819515.

"Neofusicoccum brasiliense" M.W. Marques, A.J.L. Phillips \& M.P.S. Câmara, Fungal Diversity 61: 201 (2013), nom. inval.

Sexual morph not reported. Full description and diagnosis in Marques et al. (2013b).

Marques et al. (2013b) introduced Neofusicoccum brasiliense but did not designate a holotype and thus the name was invalid. To validate the name a suitable specimen has been deposited as follows.

Type - Brazil, Pernambuco, Petrolina, Lote 1195, on Mangifera indica stems (Anacardiaceae), 2010, M.W. Marques (holotype URM 89948, culture ex-type CMM1338).

Host - Mangifera indica (Marques et al. 2013b).

Distribution - Brazil (Marques et al. 2013b).

15.7. Neofusicoccum cordaticola Pavlic, Slippers \& M.J. Wingf., Mycologia 100: 643 (2008), MycoBank MB512498.

Sexual morph not reported. See Pavlic et al. (2009) for illustrations and descriptions of asexual morph.

Type - South Africa, Kwazulu-Natal Province, Sodwana Bay, on symptomless branches and leaves, dying branches and pulp of ripe fruits of Syzygium cordatum (Myrtaceae), Mar. 2002, D. Pavlic, (holotype PREM 60066, culture ex-type CBS 123634).

Host - Syzygium cordatum (Pavlic et al. 2009).

Distribution - South Africa (Pavlic et al. 2009).

15.8. Neofusicoccum cryptoaustrale Pavlic, Maleme, Slippers \& M.J. Wingf., Persoonia 31: 271 (2013), MycoBank MB512477.

Sexual morph not reported. See Crous et al. (2013) for illustrations and descriptions of asexual morph.

Type - South Africa, Gauteng Province, Pretoria, from branches and leaves of living Eucalyptus trees (Myrtaceae), May 2005, H.M. Maleme (holotype PREM 59817, culture ex-type CBS 1122813); Gauteng Province, Eucalyptus trees, H.M. Maleme (paratype PREM 59818, culture exparatype CMW 20738, MycoBank MB512477). 
Host - Eucalyptus sp. (Crous et al. 2013).

Distribution - South Africa (Crous et al. 2013).

15.9. Neofusicoccum eucalypticola (Slippers, Crous \& M.J. Wingf.) Crous, Slippers \& A.J.L. Phillips, Stud. Mycol. 55: 248, MycoBank MB500874.

Sexual morph and asexual morph reported. See Slippers et al. (2004b) for illustrations and descriptions.

Type - Australia, Victoria, Orbost, on Eucalyptus grandis (Myrtaceae), 2001, M.J. Wingfield (holotype PREM 57848, culture ex-type CBS 115679).

Host - Eucalyptus sp. (Slippers et al. 2004b, Burgess et al. 2006).

Distribution - Australia (Slippers et al. 2004b).

15.10. Neofusicoccum eucalyptorum (Crous, H. Sm. ter. \& M.J. Wingf.) Crous, Slippers \& A.J.L. Phillips, Stud. Mycol. 55: 248. 2006. MycoBank MB500875.

Sexual morph and asexual morph reported. See Smith et al. (2001) for illustrations and descriptions.

Type - of sexual morph: South Africa, Mpumalanga, Sabie, Eucalyptus grandis, 1995, H. Smith, holotype PREM 56603; of asexual morph: South Africa, Mpumalanga, Sabie, E. grandis, 1995, H. Smith, holotype PREM 56604. The ex-type isolate was not designated in the original publication and could not be traced. Slippers et al. (2004b) regarded CBS 115791 as representative.

Hosts - Blepharocalyx salicifolius (Pérez et al. 2010), Eucalyptus spp. (Burgess et al. 2006, Smith et al. 2001, Slippers et al. 2004b, Pérez et al. 2010, De la Mora-Castaneda et al. 2014), Myrceugenia glaucescens, Myrrhinium atropurpureum var. octandrum, Syzygium cordatum (Pillay et al. 2013).

Distribution - Australia (Slippers et al. 2004b), Mexico (De la Mora-Castaneda et al. 2014), South Africa (Smith et al. 2001, Pillay et al. 2013), Uraguay (Pérez et al. 2010).

15.11. Neofusicoccum grevilleae Crous \& R.G. Shivas, Persoonia 26: 117 (2011), MycoBank MB560162.

Sexual morph not reported. See Crous et al. (2011) for illustrations and descriptions of asexual morph.

Type - Australia, Queensland, Brisbane, on leaves of Grevillea aurea (Proteaceae), 14 Jul. 2009, P.W. Crous \& R.G. Shivas (holotype CBS H-20578, culture ex-type CBS 129518).

Host - Grevillea aurea (Crous et al. 2011).

Distribution - Australia (Crous et al. 2011).

15.12. Neofusicoccum hellenicum S.F. Chen, G.Q. Li \& T.J. Michailides, Mycologia 107: 1 (2015), MycoBank MB810935.

Sexual morph not reported. See Crous et al. (2011) for illustrations and descriptions of asexual morph.

Type - Greece, Thessaloniki, from twigs of one Pistacia vera cultivar Aegina (Anacardiaceae), 23 Oct 1997, T.J. Michailides (holotype CSFF2010, culture ex-type CERC1947 = CFCC50067).

Host - Pistacia vera (Crous et al. 2011).

Distribution - Greece (Crous et al. 2011).

15.13. Neofusicoccum kwambonambiense Pavlic, Slippers \& M.J. Wingf., Mycologia 100: 643 (2008), MycoBank MB512499. 
Sexual morph not reported. See Pavlic et al. (2009) for illustrations and descriptions of asexual morph.

Type - South Africa, Kwazulu-Natal Province, Kwambonambi, on symptomless branches and leaves, dying branches and pulp of ripe fruits of Syzygium cordatum (Myrtaceae), Mar 2002, D. Pavlic (holotype PREM 60067, culture ex-type CBS 123639).

Hosts - Acacia karroo, Celtis Africana (Jami et al. 2014), Eucalyptus grandis (Pillay et al. 2013), Fragaria ananassa (Lopes et al. 2014), Syzygium cordatum (Pavlic et al. 2009, Pillay et al. 2013, Pavlic-Zupanc et al. 2015), Vitis vinifera (Abreo et al. 2013).

Distribution - Brazil (Lopes et al. 2014), South Africa (Pavlic et al. 2009, Pillay et al. 2013, Jami et al. 2014, Pavlic-Zupanc et al. 2015), Uruguay (Abreo et al. 2013).

15.14. Neofusicoccum lumnitzerae J.A. Osorio, Jol. Roux \& Z.W. de Beer, Fungal Biology (available online, http://dx.doi.org/10.1016/j.funbio.2016.09.004, 2016), MycoBank MB812012.

Sexual morph not reported. See Osorio et al. (2016) for illustrations and description.

Type - South Africa, Kwazulu-Natal Province: Kosi Bay, from asymptomatic branches of Lumnitzera racemosa (Combretaceae) J.A Osorio \& Jol. Roux. (holotype PREM 61251, culture extype CBS 139674).

Host - Lumnitzera racemosa (Osorio et al. 2016).

Distribution - South Africa (Osorio et al. 2016).

15.15. Neofusicoccum luteum (Pennycook \& Samuels) Crous, Slippers \& A.J.L. Phillips, Stud. Mycol. 55: 248 (2006), MycoBank MB500876.

Sexual morph and asexual morph reported. See Phillips et al. (2013) for illustrations and descriptions.

Type - New Zealand, Bay of Plenty, Te Puke, No 1 Road, DSIR Research Orchard, from lesions on ripe fruit of Actinidia deliciosa (Actinidiaceae), 6 Oct. 1982, S.R. Pennycook (holotype PDD 45400, culture ex-type of asexual morph PDDCC 8004). Portugal, Estremadura, Oeiras, Quinta do Marquês, on cane of Vitis vinifera cv. Galego Dourado (Vitaceae), Mar. 1996, A.J.L. Phillips (holotype of sexual morph LISE 94070, culture ex-type CBS 110299).

Hosts - Recent studies subsequent to Phillips et al. (2013) confirm the following hosts: Citrus sp. (Adesemoye et al. 2014), Erica arborea (Linaldeddu et al. 2015), Vitis vinifera (Billones-Baaijens et al. 2015, Chebil et al. 2014).

Distribution - Recent studies subsequent to Phillips et al. (2013) confirm the following distribution: California (Adesemoye et al. 2014), Italy (Linaldeddu et al. 2015), New Zealand (Billones-Baaijens et al. 2015), Tunisia (Chebil et al. 2014).

15.16. Neofusicoccum macroclavatum (T.I. Burgess, Barber \& G.E. Hardy) T.I. Burgess, Barber \& G.E. Hardy, Stud. Mycol. 55: 248 (2006), MycoBank MB500877.

Sexual morph not reported. See Burgess et al. (2005) for illustrations and descriptions of asexual morph.

Type - Australia, Western Australia, Denmark, from wood of living Eucalyptus globulus (Myrtaceae), Oct. 2002, T.I. Burgess (holotype MURU 400, culture ex-type CBS 118223).

Hosts - Eucalyptus globulus, E. saligna (Burgess et al. 2005), Vitis vinifera (Billones-Baaijens et al. 2013).

Distribution - Australia (Burgess et al. 2005), New Zealand (Billones-Baaijens et al. 2013).

15.17. Neofusicoccum mangiferae (Syd. \& P. Syd.) Crous, Slippers \& A.J.L. Phillips, Stud. Mycol. 55: 248 (2006), MycoBank MB500878. 
Sexual morph not reported. See Slippers et al. (2005) for illustrations and descriptions of asexual morph.

Type - India, Lucknow, on Mangifera indica (Anacardiaceae), F. Bahadur (E.J. Butler 1724), 22 Oct. 1908 (holotype HCIO Cultures linked to the type could not be located and probably do not exist. Slippers et al. (2005) regarded the following as representatives: CBS 118531, CBS 118532.

Hosts - Mangifera indica (Slippers et al. 2005, Ni et al. 2012, Serrato-Diaz et al. 2014), Vitis vinifera (Dissanayake et al. 2015b).

Distribution - Australia, India (Slippers et al. 2005), China (Dissanayake et al. 2015b), Puerto Rico (Serrato-Diaz et al. 2014), Taiwan (Ni et al. 2012).

15.18. Neofusicoccum mangroviorum J.A. Osorio, Jol. Roux \& Z.W. de Beer, Fungal Biology (available online, http://dx.doi.org/10.1016/j.funbio.2016.09.004, 2016), MycoBank MB814641.

Sexual morph not reported. See Osorio et al. (2016) for illustrations and description.

Type - South Africa, Kwazulu-Natal Province, Beachwood, Kosi Bay, Mtunzini, Mgazana, from asymptomatic branches of Avicennia marina, J.A Osorio \& Jol. Roux (holotype PREM 61305, culture ex-type CBS 140738).

Host - Avicennia marina, Bruguiera gymnorrhiza, Lumnitzera racemosa and Rhizophora mucronata (Osorio et al. 2016).

Distribution - South Africa (Osorio et al. 2016).

15.19. Neofusicoccum mediterraneum Crous, M.J. Wingf. \& A.J.L. Phillips, Fungal Planet, 19: 2 (2007), MycoBank MB504461.

Sexual morph not reported. See Phillips et al. (2013) for illustrations and descriptions of asexual morph.

Type - Greece, Rhodes, Rhodos Palace Hotel parking lot, on branches and leaves of Eucalyptus sp., 12 Jun. 2006, collected by P.W. Crous, M.J. Wingfield \& A.J.L. Phillips (holotype CBS H19921, culture ex-type CBS 121718).

Hosts - Recent studies subsequent to Phillips et al. (2013) confirm the following hosts: Vitis vinifera (Berraf-Tebbal et al. 2014), Diospyros kaki (Palou et al. 2013), Juglans regia (Chen et al. 2014a), Mangifera indica (Krishnapillai \& Wijeratnam 2015), Olea europaea (Úrbez-Torres et al. 2013), Pistachia vera (Michailides \& Morgan 2016, Chen et al. 2014b).

Distribution - Recent studies subsequent to Phillips et al. (2013) confirm the following distribution: Algeria (Berraf-Tebbal et al. 2014), California (Chen et al. 2014a, b, Michailides \& Morgan 2016, Úrbez-Torres et al. 2013), India (Krishnapillai et al. 2015), Spain (Palou et al. 2013).

15.20. Neofusicoccum nonquaesitum Inderb., Trouillas, Bostock \& Michailides, Mycologia 102: 1360 (2010), MycoBank MB518135.

Sexual morph not reported. See Inderbitzin et al. (2010) for illustrations and descriptions of asexual morph.

Type - USA, California, Napa County, St Helena, on cankered branch of Umbellularia californica (Lauraceae), 12 Nov. 2004, F.P. Trouillas (holotype UC1946389, culture ex-type CBS 126655).

Hosts - Malus domestica (Rooney-Latham \& Soriano 2016), Sequoiadendron giganteum (RooneyLatham et al. 2012), Umbellularia californica, Prunus dulcis (Inderbitzin et al. 2010), Vaccinium corymbosum (Espinoza et al. 2009, Pérez et al. 2014).

Distribution - Chile (Espinoza et al. 2009, Pérez et al. 2014), North America (Rooney-Latham et al. 2012), USA (California) (Inderbitzin et al. 2010, Rooney-Latham \& Soriano 2016). 
15.21. Neofusicoccum occulatum Sakalidis \& T.I. Burgess, Mol. Phylogen. Evol. 60: 340 (2011), MycoBank MB518777.

Sexual morph not reported. See Sakalidis et al. (2011) for illustrations and descriptions of asexual morph.

Type - Australia, Queensland, Karanda, symptomless branches of Eucalyptus grandis hybrid (Myrtaceae), Mar 2002, T.I. Burgess (holotype MURU467, culture ex-type CBS 128008).

Host - Eucalyptus (Sakalidis et al. 2011).

Distribution - Australia (Sakalidis et al. 2011).

15.22. Neofusicoccum parvum (Pennycook \& Samuels) Crous, Slippers \& A.J.L. Phillips, Stud. Mycol. 55: 248 (2006), MycoBank MB500879.

Sexual morph and asexual morph reported. See Phillips et al. (2013) for illustrations and descriptions.

Type - New Zealand, Bay of Plenty, Te Puke, No 3 Road, Baldwin Orchard, on small dead branch of Populus nigra (Salicaceae), 17 Dec. 1981, S.R. Pennycook (holotype PDD 45438, culture extype PDDCC 8003 = ATCC 58191).

Hosts - Plurivorous.

Distribution - Worldwide.

15.23. Neofusicoccum pennatisporum K. Taylor, Barber \& T.I. Burgess, Mycol. Res. 113: 346 (2009), MycoBank MB511826.

Sexual morph and asexual morph reported. See Taylor et al. (2009) for illustrations and descriptions. Morphologically and phylogenetically this species is divergent and its position within Neofusicoccum needs to be re-evaluated.

Type - Australia, Western Australia, Yalgorup National Park, from healthy stem of Allocasuarina fraseriana (Casuarinaceae), Jun. 2005, K.M. Taylor, holotype PERTH 07693044. WAC $13153=$ MUCC 510 (ex-type).

Host - Allocasuarina fraseriana (Taylor et al. 2009).

Distribution - Western Australia (Taylor et al. 2009).

15.24. Neofusicoccum protearum (Denman \& Crous) Crous, Slippers \& A.J.L. Phillips, Stud. Mycol. 55: 249 (2006), MycoBank MB500880.

Sexual morph and asexual morph reported. See Denman et al. (2003) for illustrations and descriptions.

Type - Of sexual morph: South Africa, Western Cape, Porterville, Baanbreek Farm, on stems of Protea magnifica (Proteaceae), 27 Jul. 1997, S. Denman (holotype PREM 57329, cultures ex-type STE-U $4361=$ CPC 4361).

Hosts - Protea sp., Leucadendron sp. (Denman et al. 2000, 2003, Marincowitz et al. 2008), Santalum acuminatum (Taylor et al. 2009).

Distribution - Australia, Portugal (continental and Madeira), South Africa, Spain (Tenerife), USA (Hawaii) (Denman et al. 2000, 2003, Marincowitz et al. 2008, Taylor et al. 2009).

15.25. Neofusicoccum ribis (Slippers, Crous \& M.J. Wingf.) Crous, Slippers \& A.J.L. Phillips, Stud. Mycol. 55: 249 (2006), MycoBank MB500881.

Sexual morph and asexual morph reported. See Slippers et al. (2004b) for illustrations and descriptions. 
Type - USA, New York, Geneva, on Ribes vulgare, 1911, J.G. Grossenbacher \& B.M. Duggar (holotype CUP-A (F.Col. 3408), culture ex-type CBS 115475).

Hosts - More than 250 hosts are listed in Farr \& Rossman (2016) but many of the reports were published before the concept of $N$. ribis was clarified by Slippers et al. (2004a) and thus are not reliable. Recent studies subsequent to Phillips et al. (2013) confirm the following hosts: Hevea brasiliensis (Ngobisa et al. 2013), Mallus domestica (Jurick et al. 2013), Psidium guajava (Nogueira Junior et al. 2016).

Distribution - Brazil (Nogueira Junior et al. 2016), Malaysia (Ngobisa et al. 2013), Pennsylvania (Jurick et al. 2013).

15.26. Neofusicoccum umdonicola Pavlic, Slippers \& M.J. Wingf., Mycologia 100: 644 (2008), MycoBank MB512500.

Sexual morph not reported. See Pavlic et al. (2009) for illustrations and descriptions of asexual morph.

Type - South Africa, Kwazulu-Natal Province, Kosi Bay from symptomless branches and leaves, dying branches and pulp of ripe fruits of Syzygium cordatum (Myrtaceae), Mar. 2002, D. Pavlic (holotype PREM 60068, culture ex-type CBS 123645).

Hosts - Schizolobium parahyba (Mehl et al. 2014), Syzygium cordatum (Pavlic et al. 2009, PavlicZupanc et al. 2015).

Distribution - Ecuador (Mehl et al. 2014), South Africa (Pavlic et al. 2009).

15.27. Neofusicoccum ursorum Pavlic, Maleme, Slippers \& M.J. Wingf., Persoonia 31: 271 (2013), MycoBank MB512478.

Sexual morph not reported. See Crous et al. (2013) for illustrations and descriptions of asexual morph.

Type - South Africa, Gauteng Province, Pretoria, from branches and leaves of living Eucalyptus trees (Myrtaceae), May 2005, H.M. Maleme (holotype PREM 59815, culture ex-type CBS 122811).

Host - Eucalyptus sp. (Crous et al. 2013).

Distribution - South Africa (Crous et al. 2013).

15.28. Neofusicoccum viticlavatum (Van Niekerk \& Crous) Crous, Slippers \& A.J.L. Phillips, Stud. Mycol. 55: 249 (2006), MycoBank MB500882.

Sexual morph not reported. See van Niekerk et al. (2004) for illustrations and descriptions of asexual morph.

Type - South Africa, Western Cape Province, Stellenbosch, on V. vinifera (Vitaceae), 2002, F. Halleen (holotype CBS H-7755, culture ex-type CBS 112878).

Host - Vitis vinifera (van Niekerk et al. 2004).

Distribution - South Africa (Western Cape Province) (van Niekerk et al. 2004).

15.29. Neofusicoccum vitifusiforme (Van Niekerk \& Crous) Crous, Slippers \& A.J.L. Phillips, Stud. Mycol. 55: 249 (2006), MycoBank MB500883.

Sexual morph not reported. See Phillips et al. (2013) for illustrations and descriptions of asexual morph.

Type - South Africa, Western Cape Province, Stellenbosch, on V. vinifera (Vitaceae), 2002, J.M. van Niekerk (holotype CBS H-7756, culture ex-type CBS 110887).

Hosts - Acacia karroo (Jami et al. 2013), Eucalyptus corticosa (as N. corticosae) (Summerell et al. 2006), Eucalyptus sp., Eucalyptus camaldulensis, Eucalyptus diversicolor, E. pauciflora, 
Eucalyptus marginata, Eucalyptus rubida, Eucalyptus viminalis (as D. eucalypti) (Barber et al. 2005, Taylor et al. 2009, Sutton 1980), Juglans regia (Chen et al. 2014a), Olea europaea (Lazzizera et al. 2008, Úrbez-Torres et al. 2013), Pistachia vera (Chen et al. 2014b), Prunus armeniaca, Prunus persica, Prunus salicina (Damm et al. 2007), Schizolobium parahyba (Mehl et al. 2014), Vaccinium corymbosum (Kong et al. 2010), Vitis vinifera (van Niekerk et al. 2004, Úrbez-Torres et al. 2012, Mondello et al. 2013).

Distribution - Australia (Sutton 1980, Barber et al. 2005, Summerell et al. 2006, Taylor et al. 2009), China (Kong et al. 2010), Italy (Lazzizera et al. 2008, Mondello et al. 2013), South Africa (van Niekerk et al. 2004, Damm et al. 2007, Jami et al. 2013, Mehl et al. 2014), USA (ÚrbezTorres et al. 2012, 2013, Chen et al. 2014a, b).

16. Neoscytalidium Crous \& Slippers, Stud. Mycol. 55: 244. 2006, MycoBank MB500868. This genus comprises three species. The sexual morph has not been reported for any species.

Type species - Neoscytalidium dimidiatum (Penz.) Crous \& Slippers.

Phillips et al. (2013) incorrectly stated that $N$. hyalinum is the type species of Neoscytalidium. The oldest epithet is dimidiatum based on Torula dimidiata (1882). Huang et al. (2016) corrected this error and placed $N$. dimidiatum as type species of the genus.

16.1. Neoscytalidium dimidiatum (Penz.) Crous \& Slippers, Stud. Mycol. 55: 244 (2006), MycoBank MB500869.

Basionym - Torula dimidiata Penz., Michelia 2: 466. 1882 (basionym; hyphomycete synanamorph).

Synonym - Neoscytalidium hyalinum (C. K. Campb. \& J. L. Mulder) AJL Phillips, Groenewald \& Crous, SIM 76: 148 (2013). See Huang et al. (2016).

Sexual morph not reported. See Crous et al. (2006) for illustrations and description of asexual morph.

Type - Holotype of basionym apparently lost. Since $N$. hyalinum and $N$. dimidiatum are considered to be synonyms, the isotype and ex-isotype culture of $N$. hyalinum have been used as the standard for this species. Lectotype: United Kingdom, sole of human foot, 20 Nov. 1973, C.K. Campbell (isotype CBS H-7745, culture ex-isotype CBS 145.78).

Hosts - Recent studies subsequent to Phillips et al. (2013) confirm the following hosts: Jatropha curcas (Machado et al. 2014a), Manihot esculenta (Machado et al. 2014b), Vitis vinifera (Correia et al. 2016b).

Distribution - Recent studies subsequent to Phillips et al. (2013) confirm the following distribution: Brazil (Machado et al. 2014a, b, Correia et al. 2016b).

16.2. Neoscytalidium novaehollandiae Pavlic, T.I. Burgess \& M.J. Wingf., Mycologia 100: 862 (2008), MycoBank MB512103.

Sexual morph not reported. See Pavlic et al. (2008) for illustrations and descriptions of asexual morph.

Type - Australia, Western Australia, Bell Gorge, on Crotalaria medicaginea (Fabaceae), Jul. 2006, T.I. Burgess (holotype PREM 60069, culture ex-type CBS 122071).

Hosts - Acacia synchronica, Adansonia gibbosa, Crotalaria medicaginea, Grevillia agrifolia (Pavlic et al. 2008), Mangifera indica, Ficus carica (Ray et al. 2010).

Distribution - Australia (Pavlic et al. 2008, Ray et al. 2010).

16.3. Neoscytalidium orchidacearum S. K. Huang, N. Tangthirasunun, J. C. Kang \& K. D. Hyde, Mycobiology 44: 79 (2016).

Sexual morph not reported. See Huang et al. (2016) for illustrations and descriptions of asexual morph. 
Type - Thailand, Sukhotai, on dead leaves of Orchid (Orchidaceae), 5 Aug 2012; S Hongsanan (holotype MFLU 13-0294, cultures ex-type MFLUCC 12-0533 and GZUCC 15113001).

Host - Orchid (Huang et al. 2016).

Distribution - Thailand (Huang et al. 2016).

17. Phaeobotryon Theiss. \& Syd., Ann. Mycol. 13: 664 (1915), MycoBank MB3892.

This genus comprises four species. Sexual and asexual morphs have been reported within the genus. No cultures of the type species are extant.

Type species - Phaeobotryon cercidis (Cooke) Theiss. \& Syd., Ann. Mycol. 13: 664. 1915.

17.1. Phaeobotryon cupressi Abdollahz., Zare \& A.J.L. Phillips, Persoonia 23: 6 (2009), MycoBank MB513236.

Sexual morph not reported. See Phillips et al. (2013) for illustrations and descriptions of asexual morph.

Type - Iran, Golestan Province, Gorgan, City Park, on twigs of Cupressus sempervirens (Cupressaceae), 15 Aug. 2006, M.A. Aghajani (holotype IRAN 13940F, cultures ex-type IRAN $1455 \mathrm{C}=\mathrm{CBS} 124700$ ).

Hosts - Cupressus sempervirens (Abdollahzadeh et al. 2009, Zlatkovic et al. 2016), Juniperus scopulorum (Alves et al. 2013).

Distribution - Iran (Abdollahzadeh et al. 2009), USA (Alves et al. 2013), Western Balkans (Zlatkovic et al. 2016).

17.2. Phaeobotryon mamane Crous \& A.J.L. Phillips, Persoonia 21: 45 (2008), MycoBank MB506581.

Sexual morph and asexual morph reported. See Phillips et al. (2008) for illustrations and descriptions.

Type - USA, Hawaii, Manna Koa Park, Saddle Road, on stems of Sophora chrysophylla (Fabaceae), Jul. 2005, W. Gams (holotype CBS H-20109, culture ex-type CBS 122980).

Host - Sophora chrysophylla (Phillips et al. 2008).

Distribution - USA (Hawaii) (Phillips et al. 2008).

17.3. Phaeobotryon negundinis Daranagama, Bulgakov \& K.D. Hyde, Mycosphere 7: 936 (2016), Facesoffunginumber FoF01916.

Sexual morph not reported. See Daranagama et al. (2016) for illustrations and descriptions of asexual morph.

Type - Russia, Rostov region, Rostov-on-Don city, Botanical Garden of Southern Federal University, Higher Park, on dying and dead twigs and branches of Acer negundo L. (Sapindaceae), 05 March 2014, T.S. Bulgakov (holotype MFLU 16-0475, culture ex-type MFLUCC 15-0436).

Hosts - Acer negundo, Ligustrum vulgare, Forsythia $\times$ intermedia (Daranagama et al. 2016).

Distribution - Russia (Daranagama et al. 2016).

17.4. Phaeobotryon rhois C.M. Tian, X.L. Fan \& K.D. Hyde, Phytotaxa 205: 95 (2015), MycoBank MB811599, Facesoffunginumber FOF00596.

Sexual morph not reported. See Fan et al. (2015) for illustrations and descriptions of asexual morph.

Type - China, Ningxia Province: Yinchuan City, Yongning County, Wanghong Town, Nongsheng Village, $38^{\circ} 22^{\prime} 97.02^{\prime \prime} \mathrm{N}, 106^{\circ} 21^{\prime} 44.89^{\prime}$ " E, alt. $1101 \mathrm{~m}$, on twigs and branches of Rhus typhina 
(Anacardiaceae), 26 July 2013, Xin-lei Fan \& Hong Fan (holotype BJFC-S1007, cultures ex-type CFCC 89662 = CCTCC AF2014017).

Host - Rhus typhina (Fan et al. 2015).

Distribution - China (Fan et al. 2015).

18. Pseudofusicoccum Mohali, Slippers \& M.J. Wingf., Stud. Mycol. 55: 249 (2006), MycoBank MB500884.

This genus comprises seven species. The sexual morph has not been reported for any species. The phylogenetic position of this genus has been questioned (Slippers et al. 2013) and it is still uncertain if it resides within Botryosphaeriaceae. However, until its position has been definitively resolved we continue to regard it as a genus within Botryophaeriaceae.

Type species - Pseudofusicoccum stromaticum (Mohali, Slippers \& M.J. Wingf.) Mohali, Slippers \& M.J. Wingf., Stud. Mycol. 55: 249. 2006.

18.1. Pseudofusicoccum adansoniae Pavlic, T.I. Burgess \& M.J. Wingf., Mycologia 100: 855 (2008), MycoBank MB512048.

Sexual morph not reported. See Pavlic et al. (2008) for illustrations and descriptions of asexual morph.

Type - Australia, Western Australia, Derby, on Adansonia gibbosa (Malvaceae), Jul. 2006, T.I. Burgess (holotype PREM 59841, culture ex-type CBS 122055).

Hosts - Adansonia gibbosa, Acacia synchronica, Eucalyptus sp., Ficus opposita (Pavlic et al. 2008), Adansonia gregorii, Grevillea agrifolia (Sakalidis et al. 2011), Cassia fistula (Trakunyingcharoen et al. 2015b), Dimocarpus longan (Trakunyingcharoen et al. 2015b), Hevea brasiliensis (Trakunyingcharoen et al. 2015a), Jatropha podagrica (Sharma et al. 2013), Senna siamea (Trakunyingcharoen et al. 2015b), Tectona grandis (Doilom et al. 2015).

Distribution - Australia (Pavlic et al. 2008, Sakalidis et al. 2011), India (Sharma et al. 2013), Thailand (Doilom et al. 2015, Trakunyingcharoen et al. 2015a, b).

18.2. Pseudofusicoccum ardesiacum Pavlic, T.I. Burgess \& M.J. Wingf., Mycologia 100: 858 (2008), MycoBank MB512051.

Sexual morph not reported. See Pavlic et al. (2008) for illustrations and descriptions of asexual morph.

Type - Australia, Western Australia, Mount Hardman, Great Northern Highway, on Adansonia gibbosa (Malvaceae), Jul. 2006, T.I. Burgess (holotype PREM 59843, culture ex-type CBS 122062).

Hosts - Adansonia gibbosa, Eucalyptus sp. (Pavlic et al. 2008), Caesalpinia pulcherrima, Veitchia merrillii (Trakunyingcharoen et al. 2015b).

Distribution - Australia (Pavlic et al. 2008), Thailand (Trakunyingcharoen et al. 2015b).

18.3. Pseudofusicoccum artocarpi T. Trakunyingcharoen, L. Lombard \& Crous, Persoonia 34: 96 (2014), MycoBank MB810167.

Sexual morph not reported. See Trakunyingcharoen et al. (2015b) for illustrations and descriptions of asexual morph.

Type - Thailand, Chiang Mai province, on twigs of Artocarpus heterophyllus (Moraceae), May 2012, T. Trakunyingcharoen (holotype CBS H-21935, culture ex-type CBS 138655).

Host - Artocarpus heterophyllus (Trakunyingcharoen et al. 2015b).

Distribution - Thailand (Trakunyingcharoen et al. 2015b). 
18.4. Pseudofusicoccum kimberleyensis Pavlic, T.I. Burgess \& M.J. Wingf., Mycologia 100: 857 (2008), MycoBank MB512049.

Sexual morph not reported. See Pavlic et al. (2008) for illustrations and descriptions of asexual morph.

Type - Australia, Western Australia, Tunnel Creek National Park, on Acacia synchronica (Fabaceae), Jul. 2006, T.I. Burgess (holotype PREM 59842, culture ex-type CBS 122058).

Hosts - Adansonia gibbosa, Acacia synchronica, Eucalyptus sp., Ficus opposita (Pavlic et al. 2008).

Distribution - Western Australia (Pavlic et al. 2008).

18.5. Pseudofusicoccum olivaceum Mehl \& Slippers, Mycologia 103: 537 (2011), MycoBank MB513501.

Sexual morph not reported. See Mehl et al. (2011) for illustrations and descriptions of asexual morph.

Type - South Africa, Mpumalanga Province, Kruger National Park, Pretoriuskop, on an asymptomatic branch of Pterocarpus angolensis (Fabaceae), Sep. 2005, J. Roux (holotype PREM 60328, culture ex-type CBS 124939).

Host - Pterocarpus angolensis (Mehl et al. 2011).

Distribution - South Africa (Mehl et al. 2011).

18.6. Pseudofusicoccum stromaticum (Mohali, Slippers \& M.J. Wingf.) Mohali, Slippers \& M.J. Wingf., Stud. Mycol. 55: 249, MycoBank MB500885.

Sexual morph not reported. See Phillips et al. (2013) for illustrations and descriptions of asexual morph.

Type - Venezuela, Portuguesa Morph, Acarigua, Smurfit Company, on branches of Eucalyptus urophylla (Myrtaceae), Feb. 2003, S. Mohali (holotype PREM 58237, culture ex-type CMW 13366, other representative cultures CBS 117448, CBS 117449).

Hosts - Eucalyptus sp. (Mohali et al. 2006), Acacia mangium (Mohali et al. 2006), Mangifera indica (Marques et al. 2012, 2013b).

Distribution - Brazil (Marques et al. 2012, 2013b), Venezuela (Mohali et al. 2006).

18.7. Pseudofusicoccum violaceum Mehl \& Slippers, Mycologia 103: 541 (2011), MycoBank MB513500.

Sexual morph not reported. See Mehl et al. (2011) for illustrations and descriptions of asexual morph.

Type - South Africa, Mpumalanga Province, Mawewe Nature Reserve, on an asymptomatic branch of Pterocarpus angolensis (Fabaceae), Dec. 2005, J.W.M. Mehl \& J. Roux (holotype PREM 60333, culture ex-type CBS 124936).

Hosts - Pterocarpus angolensis (Mehl et al. 2011), Tinospora cordifolia (Mishra et al. 2012).

Distribution - India (Mishra et al. 2012), South Africa (Mehl et al. 2011).

19. Sakireeta Subram. \& K. Ramakr., Journal of the Indian Botanical Society 36: 83 (1957).

This genus comprises only one species. The sexual morph has not been reported.

Type species - Sakireeta madreeya Subram. \& K. Ramakr 1957.

19.1. Sakireeta madreeya Subram. \& K. Ramakr., Journal of the Indian Botanical Society 36: 84 (1957). 
Synonym - Tiarosporella madreeya (Subram. \& K. Ramakr.) Nag Raj, Canadian Journal of Botany 51: 2470 (1974) [1973].

Sexual morph not reported. See Crous et al. (2015a) for illustrations and descriptions of asexual morph.

Type - India, Madras, Choolai, on dead culm of Aristida setacea (Poaceae), 27 Sept. 1951, K. Ramakrishnan (holotype MUBL 631); Kurukshetra Univ., undetermined grass host, July 1976, R.S. Mehrotra, CBS H-21854, culture CBS 532.76.

Host - Aristida setacea (Crous et al. 2015a).

Distribution - India (Crous et al. 2015a).

20. Sardiniella Linaldeddu, A. Alves \& A.J.L. Phillips, Mycosphere 7: 900 (2016), MycoBank MB817511.

Sardiniella was introduced to accommodate species that are morphologically similar to Diplodia and Dothiorella/Spencermartinsia. Phylogenetically Sardiniella is closely related to Neofusicoccum and Dothiorella/Spencermartinsia. This genus comprises a single species. The sexual morph has not been reported.

Type species - Sardiniella urbana Linaldeddu, A. Alves \& A.J.L. Phillips 2016.

20. 1. Sardiniella urbana Linaldeddu, A. Alves \& A.J.L. Phillips, Mycosphere 7: 900 (2016), MycoBank MB817512.

Sexual morph not reported. See Linaldeddu et al. (2016b) for illustrations and descriptions of asexual morph.

Type - Italy, Sassari, isolated from a branch canker of Celtis australis (Cannabaceae), 9 September 2013, Benedetto T. Linaldeddu (holotype LISE 96308, culture ex-type CBS 141580).

Host - Celtis australis (Linaldeddu et al. 2016b)

Distribution - Italy (Linaldeddu et al. 2016b)

21. Spencermartinsia A.J.L. Phillips, A. Alves \& Crous, Persoonia 21: 51 (2008), MycoBank MB511762.

This genus comprises eight species (Fig. 4). Sexual morph and asexual morphs have been reported within the genus. Phylogenetically Spencermartinsia is very closely related to Dothiorella. Morphologically the two can be differentiated only in the sexual morphs; ascospores of Spencermartinsia bear hyaline apiculi, which are absent in Dothiorella. With the addition of more species, the distinction between these two genera has become more tenuous. Nevertheless, in this paper we continue to regard them as separate pending further study.

Type species - Spencermartinsia viticola (A.J.L. Phillips \& J. Luque) A.J.L. Phillips, A. Alves \& Crous, Persoonia 21: 51. 2008.

21.1. Spencermartinsia alpina Y. Zhang ter. \& M. Zhang, sp. nov., MycoBank MB 819517.

“Spencermartinsia alpina” Y. Zhang ter. \& M. Zhang, Mycosphere 7: 945 (2016), nom. inval.

Zhang et al. (2016) introduced Spencermartinsia alpina as a new species but did not explicitly designate a holotype and thus the name was invalid. To validate the name the holotype is cited below. The sexual morph was not reported. Full description and diagnosis in Zhang et al. (2016).

Type - China, Yunnan Province, Chuxiong City, Zixi Mountain Forest Park, from thick branch of dead young tree of Platycladus orientalis (Cupressaceae), 16 Dec 2014, leg. W. He \& J.R. Wu, det. Y. Zhang (holotype HMAS254733, culture ex-type CGMCC 3.18001).

Host - Platycladus orientalis (Zhang et al. 2016). 
Distribution - China (Zhang et al. 2016).

21.2. Spencermartinsia citricola A.J.L. Phillips \& Abdollahz., Persoonia 32: 7 (2014), MycoBank MB803992.

Sexual morph not reported. See Abdollahzadeh et al. (2014) for illustrations and descriptions of asexual morph.

Type - New Zealand, Northland, Kerikeri, Collins Orchard, Inlet Road, on twigs of Citrus sinensis (Rutaceae), Sept. 2006, S.R. Pennycook, P.R. Johnston and B.C. Paulus (holotype PDD92023, cultures ex-type ICMP16828 = CBS124729).

Host - Citrus sinensis (Abdollahzadeh et al. 2014).

Distribution - New Zealand (Abdollahzadeh et al. 2014).

21.3. Spencermartinsia mangiferae Abdollahz., Javadi \& A.J.L. Phillips, Persoonia 32: 9 (2014), MycoBank MB803993.

Sexual morph not reported. See Abdollahzadeh et al. (2014) for illustrations and descriptions of asexual morph.

Type - Iran, Hormozgan Province, Bandar Abbas (Hajiabad-Siaho), on twigs of Mangifera indica (Anacardiaceae), Mar. 2007, J. Abdollahzadeh \& A. Javadi (holotype IRAN 14266F, cultures extype IRAN 1584C = CBS 124727).

Host - Mangifera indica (Abdollahzadeh et al. 2014).

Distribution - Iran (Abdollahzadeh et al. 2014).

21.4. Spencermartinsia plurivora Abdollahz., Javadi \& A.J.L. Phillips, Persoonia 32: 9 (2014), MycoBank MB803994.

Sexual morph not reported. See Abdollahzadeh et al. (2014) for illustrations and descriptions of asexual morph.

Type - Iran, Khuzestan Province, Dezful (Safiabad Citrus Research Centre), on twigs of Citrus sp. (Rutaceae), Nov. 2006, J. Abdollahzadeh \& A. Javadi (holotype IRAN 14267F, cultures ex-type IRAN $1557 \mathrm{C}=$ CBS 124724).

Host - Citrus sp. (Abdollahzadeh et al. 2014).

Distribution - Iran (Abdollahzadeh et al. 2014).

21.5. Spencermartinsia rosulata F.J.J. Van der Walt, Slippers \& G.J. Marais, Persoonia 33: 164 (2014), MycoBank MB518724.

Sexual morph not reported. See Slippers et al. (2014) for illustrations and descriptions of asexual morph.

Type - Namibia, Windhoek, from Acacia karroo (Fabaceae), Feb. 2006, F.J.J. van der Walt \& J. Roux (holotype PREM 59622, culture ex-type CBS 121760).

Host - Acacia sp. (Slippers et al. 2014).

Distribution - Namibia, South Africa (Slippers et al. 2014).

21.6. Spencermartinsia viticola (A.J.L. Phillips \& J. Luque) A.J.L. Phillips, A. Alves \& Crous, Persoonia 21: 51 (2008), MycoBank MB511763.

Sexual morph and asexual morph reported. See Phillips et al. (2013) for illustrations and descriptions.

Type - Spain, Catalonia, Vimbodí, near the Monastery of Poblet, on pruned canes of Vitis vinifera cv. Garnatxa Negra (Vitaceae), Aug. 2004, J. Luque \& S. Martos (holotype LISE 95177, culture ex-type CBS 117009). 
Hosts - Acacia karroo (Jami et al. 2013, 2014), Celtis africana (Jami et al. 2014), Citrus sp. (Adesemoye \& Eskalen 2011, Inderbitzin et al. 2010), Gymnosporia buxifolia (Jami et al. 2014), Populus cathayana (Zhang et al. 2009), Poniciana gilliesii (Phillips et al. 2008), Prunus persica and P. salicina (Damm et al. 2007), Vitis vinifera (de Wet et al. 2009, Luque et al. 2005, Qiu et al. 2011, Úrbez-Torres et al. 2007, Pitt et al. 2013b, Diaz et al. 2013, Valencia et al. 2015).

Distribution - Australia (Qiu et al. 2011, Pitt et al. 2013b), Chile (Diaz et al. 2013), China (Zhang et al. 2009, Valencia et al. 2015), France (Phillips et al. 2008), South Africa (Damm et al. 2007, de Wet et al. 2009, Jami et al. 2013, 2014), Spain (Luque et al. 2005) and USA (Adesemoye \& Eskalen 2011, Inderbitzin et al. 2010, Úrbez-Torres et al. 2007).

21.7. Spencermartinsia westrale W.M. Pitt, J.R. Úrbez-Torres \& Trouillas, Australas. Pl. Path. 44: 48 (2015), MycoBank MB808286.

Sexual morph not reported. See Pitt et al. (2015) for illustrations and descriptions of asexual morph.

Type - Australia, Upper Swan, Western Australia, discarded canes of Vitis vinifera (Vitaceae), November 2009, F.P. Trouillas (holotype, DAR80529, culture ex-type, DAR80529).

Host - Vitis vinifera (Pitt et al. 2015).

Distribution - Australia (Pitt et al. 2015).

21.8. Spencermartinsia yunnana Y. Zhang ter. \& M. Zhang, sp. nov, MycoBank MB 819518.

“Spencermartinsia yunnana” Y. Zhang ter. \& M. Zhang, Mycosphere 7: 946 (2016), nom. inval.

Zhang et al. (2016) introduced Spencermartinsia yunnana as a new species but did not explicitly designate a holotype and thus the name was invalid. To validate the name the holotype is cited below. The sexual morph not reported. Full description and diagnosis in Zhang et al. (2016).

Type - China, Yunnan Province, Chuxiong City, Zixi Mountain Forest Park, from dead branch of Camellia sp. (Theaceae), 16 Dec 2014, leg. W. He \& J.R. Wu, det. Y. Zhang (holotype HMAS254734, culture ex-type CGMCC 3.17999).

Hosts - Acer buergerianum, Camellia sp., Poncirus trifoliata, Ternstroemia gymnanthera (Zhang et al. 2016).

Distribution - China (Zhang et al. 2016).

22. Sphaeropsis Sacc., Michelia 2: 105. 1880, MycoBank MB9992.

This genus comprises five species. Sexual and asexual morphs have been reported within the genus. Type species - Sphaeropsis visci (Alb. \& Schwein.) Sacc., Michelia 2: 105. 1880.

22.1. Sphaeropsis citrigena (A.J.L. Phillips, P.R. Johnst. \& Pennycook) A.J.L. Phillips \& A. Alves, Stud. Mycol. 76: 158 (2013), MycoBank MB805463.

Sexual morph and asexual morph reported. See Phillips et al. (2008) and Phillips et al. (2013) for illustrations and descriptions.

Type - New Zealand, Northland, Kerikeri, Davies Orchard (\#2), Inlet Road, on recently dead barkcovered twigs of Citrus sinensis (Rutaceae), 6 Sep. 2006, S.R. Pennycook, P.R. Johnston \& B.C. Paulus (holotype PDD 89238, culture ex-type ICMP 16812).

Host - Citrus sinensis (Phillips et al. 2013).

Distribution - New Zealand (Phillips et al. 2013).

22.2. Sphaeropsis eucalypticola (Doilom, J.K. Liu, \& K.D. Hyde) A.J.L. Phillips, Stud. Mycol. 76: 158 (2013), MycoBank MB805464. 
Asexual morph not reported. See Liu et al. (2012) and Phillips et al. (2013) for illustrations and descriptions of sexual morph.

Type - Thailand, Chiang Rai Province, Muang District, on dead twig of Eucalyptus sp. (Myrtaceae), 8 Aug. 2011, M. Doilom (holotype MFLU 12-0753, culture ex-type MFLUCC 11$0579=$ CBS 133993).

Hosts - Eucalyptus sp. (Liu et al. 2012), Tectona grandis (Doilom et al. 2015).

Distribution - Thailand (Liu et al. 2012, Doilom et al. 2015).

22.3. Sphaeropsis porosa (Van Niekerk \& Crous) A.J.L. Phillips \& A. Alves, Stud. Mycol. $76: 159$ (2013), MycoBank MB805465.

Sexual morph not reported. See Phillips et al. (2013) for illustrations and descriptions of asexual morph.

Type - South Africa, Western Cape Province, Stellenbosch, on Vitis vinifera (Vitaceae), 2002, J.M. van Niekerk (holotype CBS H-12039, culture ex-type CBS 110496).

Host - Vitis vinifera (van Niekerk et al. 2004).

Distribution - South Africa (Western Cape Province) (van Niekerk et al. 2004).

22.4. Sphaeropsis variabilis F.J.J. Van der Walt, Slippers \& G.J. Marais, Persoonia 33: 164 (2014), MycoBank MB518720.

Sexual morph not reported. See Slippers et al. (2014) for illustrations and description of asexual morph. In our families tree (Fig. 1) S. variabilis appears to be more closely related to Alanphillipsia than to Sphaeropsis. In the phylogenies presented by Slippers et al. (2014) S. variabilis was not clearly aligned within Sphaeropsis, but they did not include any species of Alanphillipsia in their phylogenies. Furthermore, Slippers et al. (2014) did not include any details of paraphyses, which are an important character that distinguishes Sphaeropsis from Diplodia. For these reasons we consider that the position of $S$. variabilis in Sphaeropsis is tenable and needs to be re-assessed.

Type - Namibia, Windhoek, from Acacia karroo (Fabaceae), Feb. 2006, F.J.J. van der Walt \& J. Roux (holotype PREM 59637, culture ex-type CBS 121774).

Host - Acacia sp. (Slippers et al. 2014).

Distribution - Namibia, South Africa (Slippers et al. 2014).

22.5. Sphaeropsis visci (Alb. \& Schwein.) Sacc., Michelia 2: 105. (1880), MycoBank MB281898.

Sexual morph and asexual morph reported. See Phillips et al. (2008) and Phillips et al. (2013) for illustrations and descriptions.

Type - Germany, on Viscum album (Santalaceae), Albertini \& Schweinitz, holotype could not be located and presumably lost. Ukraine, National Nature Park 'Svjatie Gory', on branches of Viscum album, 10 Mar. 2007, Á. Akulov (neotype CWU (MYC) AS 2271, cultures ex-neotype CBS 122526, CBS 122527).

Hosts - Viscum album (Sutton 1980, Phillips et al. 2008, Varga et al. 2014, Poczai et al. 2015, Zlatkovic et al. 2016).

Distribution - Austria, Czechoslovakia, Egypt, Romania (Sutton 1980), Ukraine (Phillips et al. 2008), Hungary (Poczai et al. 2015), Serbia, Luxemburg (Zlatkovic et al. 2016).

23. Tiarosporella Höhn, Ber. Deutsch. Bot. Ges. 37: 159. (1919), MycoBank MB10233.

This genus comprises only one species. The sexual morph has not been reported.

Type species: Tiarosporella paludosa (Sacc. \& Fiori ex P. Syd.) Höhn 1919. 
23.1. Tiarosporella paludosa (Sacc. \& Fiori) Höhn., Berichte der Deutschen Botanischen Gesellschaft 37: 159 (1919).

Basionym - Neottiospora paludosa Sacc. \& Fiori, Hedwigia Beiblätter 38: 137 (1899).

Sexual morph not reported. See Crous et al. (2015a) for illustrations and descriptions of asexual morph.

Type - Germany, Berlin, Zahlendorf, on leaves of Eriophorum polystachium (Cyperaceae), Oct. 1895, P. Sydow (holotype in FH, isotype DAOM 130546). Netherlands, Winterswijk in the Korenburgerveen, Latitude 51.990133, Longitude 6.664013, on Trichophorum cespitosum subsp. germanicum, 28 Apr. 2013, W. Quaedvlieg (epitype CBS H-21855 MBT200481, culture ex-epitype CBS 138577).

Hosts - Eleocharis palustris, Trichophorum cespitosum (Crous et al. 2015a).

Distribution - Germany, Netherlands (Crous et al. 2015a).

\section{Acknowledgements}

We are grateful to the CARS-30, Youth Foundation of Beijing Academy of Agriculture and Forestry Sciences (number QNJJ 201515). K.D. Hyde thanks the Chinese Academy of Sciences, project number 2013T2S0030, for the award of Visiting Professorship for Senior International Scientists at Kunming Institute of Botany. AJL Phillips acknowledges support from Biosystems and Integrative Sciences Institute (BioISI, FCT/UID/ Multi/04046/2013).

\section{References}

Abdollahzadeh J, Javadi A, Mohammadi Goltapeh E, Zare R, Phillips AJL. 2010 - Phylogeny and morphology of four new species of Lasiodiplodia from Iran. Persoonia 25, 1-10.

Abdollahzadeh J, Javadi A, Zare R, Phillips AJL. 2014 - A phylogenetic study of Dothiorella and Spencermatinsia species associated with woody plants in Iran, New Zealand, Portugal and Spain. Persoonia 32, 1-12.

Abdollahzadeh J, Mohammadi Goltapeh E, Javadi A, Shams-Bakhsh M, Zare R, et al. 2009 Barriopsis iraniana and Phaeobotryon cupressi: two new species of the Botryosphaeriaceae from trees in Iran. Persoonia 23, 1-8.

Abdollahzadeh J, Zare R, Phillips AJL. 2013 - Phylogeny and taxonomy of Botryosphaeria and Neofusicoccum species in Iran, with description of Botryosphaeria scharifii sp. nov. Mycologia 105, 210-220.

Abreo E, Martinez S, Bettucci L, Lupo S. 2013 - Characterization of Botryosphaeriaceae species associated with grapevines in Uruguay. Australasian Plant Pathology 42, 241-249.

Acimovic SG, Harmon CL, Bec S, Wyka S, Broders K, et al. 2016 - First report of Diplodia corticola causing decline of red oak (Quercus rubra) trees in Maine. Plant Disease 100, 649-650.

Adamcik S, Cai L, Chakraborty D, Chen XH, Cotter HVT, et al. 2015 - Fungal Biodiversity profiles 1-10. Cryptogamie Mycologie 36, 121-166.

Adamson K, Klavina D, Drenkhan R, Gaitnieks T, Hanso M. 2015 - Diplodia sapinea is colonizing the native Scots pine (Pinus sylvestris) in the northern Baltics. European Journal of Plant Pathology 143, 343-350.

Adesemoye AO, Eskalen A. 2011 - First report of Spencermartinsia viticola, Neofusicoccum austral \& N. parvum causing branch canker of citrus in California. Plant Disease 95, 770.

Adesemoye AO, Mayorquin JS, Wang DH, Twizeyimana M, Lynch SC, et al. 2014 - Identification of species of Botryosphaeriaceae causing bot gummosis in citrus in California. Plant Disease 98, 55-61.

Akgul DS, Savas NG, Teker T, Keykubat B, Mayorquin JS. 2015 - Fungal trunk pathogens of sultana seedless vineyards in Aegean region of Turkey. Phytopathologia Mediterranea 54, 380-393. 
Al-Sadi AM, Al-Ghaithi AG, Al-Fahdi N, Al-Yahyai R. 2014 - Characterization and pathogenicity of fungal pathogens associated with root diseases of citrus in Oman. International Journal of Agriculture and Biology 16, 371-376.

Al-Sadi AM, Al-Wehaibi AN, Al-Shariqi RM, Alhammadi MS, Alhosni IA, et al. 2013 Population genetic analysis reveals diversity in Lasiodiplodia species infecting date palm, citrus, and mango in Oman and the UAE. Plant Disease 97, 1363-1369.

Alves A, Barradas C, Phillips AJL, Correia A. 2013 - Diversity of Botryosphaeriaceae species associated with conifers in Portugal. European Journal of Plant Pathology 135, 791-804.

Alves A, Correia A, Luque J, Phillips AJL. 2004 - Botryosphaeria corticola, sp. nov. on Quercus species, with notes and description of Botryosphaeria stevensii and its anamorph, Diplodia mutila. Mycologia 96, 598-613.

Alves A, Correia A, Phillips AJL. 2006 - Multi-gene genealogies and morphological data support Diplodia cupressi $\mathrm{sp}$. nov., previously recognized as $D$. pinea f. sp. cupressi, as a distinct species. Fungal Diversity 23, 1-15.

Alves A, Crous PW, Correia A, Phillips AJL. 2008 - Morphological and molecular data reveal cryptic speciation in Lasiodiplodia theobromae. Fungal Diversity 28, 1-13.

Alves A, Linaldeddu BT, Deidda A, Scanu B, Phillips AJL. 2014 - The complex of Diplodia species associated with Fraxinus and some other woody hosts in Italy and Portugal. Fungal Diversity 67, 143-156.

Ammad F, Benchabane M, Toumi M, Belkacem N, Guesmi A, et al. 2014 - Occurrence of Botryosphaeriaceae species associated with grapevine dieback in Algeria. Turkish Journal of Agriculture and Forestry 38, 865-876.

Andolfi A, Basso S, Giambra S, Conigliaro G, Piccolo SL, et al. 2016 - Lasiolactols A and B produced by the grapevine fungal pathogen Lasiodiplodia mediterranea. Chemistry \& Biodiversity 13, 395-402.

Ariyawansa HA, Hyde KD, Jayasiri SC, Buyck B, Cui YY, et al. 2015 - Fungal Diversity Notes 111-252 Taxonomic and phylogenetic contributions to fungal taxa. Fungal Diversity 75, $27-274$.

Ariyawansa HA, Hyde KD, Liu JK, Wu SP, Liu ZY. 2016 - Additions to Karst Fungi 1: Botryosphaeria minutispermatia sp. nov., from Guizhou Province, China. Phytotaxa 275, 035-044.

Barber PA, Burgess TJ, Hardy GEStJ, Slippers B, Keane PJ, et al. 2005 - Botryosphaeria species from Eucalyptus in Australia are pleoanamorphic, producing Dichomera synanamorphs in culture. Mycological Research 109, 1347-1363.

Barradas C, Correia A, Alves A. 2013 - First report of Neofusicoccum australe and N. luteum associated with canker and dieback of Quercus robur in Portugal. Plant Disease 97, 560.

Begoude BAD, Slippers B, Wingfield MJ, Roux J. 2011 - The pathogenic potential of endophytic Botryosphaeriaceous fungi on Terminalia species in Cameroon. Forest Pathology 41, 281292.

Berraf-Tebbal A, Guereiro MA, Phillips AJL. 2014 - Phylogeny of Neofusicoccum species associated with grapevine trunk diseases in Algeria, with description of Neofusicoccum algeriense sp. nov. Phytopathologia Mediterranea 53, 416-427.

Bian CH, Miao P, Kang YB. 2015 - First report of canker disease of flue-cured tobacco caused by Botryosphaeria dothidea in China. Plant Disease 99, 890-890.

Billones-Baaijens R, Jones EE, Ridgway HJ, Jaspers MW. 2013 - Virulence affected by assay parameters during grapevine pathogenicity studies with Botryosphaeriaceae nursery isolates. Plant Pathology 62, 1214-1225.

Billones-Baaijens R, Ridgway HJ, Jones EBG, Jaspers MV. 2015 - Spatial distribution of Neofusicoccum species within a rootstock mother vine indicates potential infection pathways. European Journal of Plant Pathology 141, 267-269. 
Burgess TI, Barber PA, Hardy GEStJ. 2005 - Botryosphaeria spp. Associated with eucalypts in Western Australia, including the description of Fusicoccum macroclavatum sp. nov. Australasian Plant Pathology 34, 557-567.

Burgess TI, Barber PA, Mohali S, Pegg G, Beer W de, et al. 2006 - Three new Lasiodiplodia spp. from the tropics, recognized based on DNA sequence comparisons and morphology. Mycologia 98, 423-435.

Carlucci A, Cibelli F, Lops F, Raimondo ML. 2015 - Characterization of Botryosphaeriaceae species as causal agents of trunk disease on grapevines. Plant Disease 99, 1678-1688.

Castillo S, Borrero C, Castano R, Rodríguez A, Avilés M. 2013 - First report of canker disease caused by Neofusicoccum parvum and $N$. australe on blueberry bushes in Spain. Plant Disease 97, 1112-1112.

Castro-Medina F, Mohali SR, Úrbez-Torres JR, Gubler WD. 2014 - First report of Lasiodiplodia pseudotheobromae causing trunk cankers in Acacia mangium in Venezuela. Plant Disease 98, 686-686.

Chebil S, Fersi R, Yakoub A, Chenenaoui S, Chattaoui M, et al. 2014 - First report of Botryosphaeria dothidea, Diplodia seriata, and Neofusicoccum luteum associated with canker and dieback of grapevines in Tunisia. Plant Disease 98, 420-420.

Chen SF, Fichtner E, Morgan DP, Michailides TJ. 2013a - First report of Lasiodiplodia citricola and Neoscytalidium dimidiatum causing death of graft union of English walnut in California. Plant Disease 97, 993-993.

Chen SF, Li G, Liu F, Michailides TJ. 2015 - Novel species of Botryosphaeriaceae associated with shoot blight of pistachio. Mycologia 107, 780-792.

Chen SF, Morgan DP, Hasey JK, Michailides TJ. 2013b - First report of Lasiodiplodia citricola associated with stem canker of peach in California, USA. Journal of Plant Pathology 95, 659-659.

Chen SF, Morgan DP, Hasey JK, Anderson K, Michailides TJ. 2014a - Phylogeny, morphology, distribution, and pathogenicity of Botryosphaeriaceae and Diaporthaceae from English walnut in California. Plant Disease 98, 636-652.

Chen SF, Morgan DP, Michailides TJ. 2014b - Botryosphaeriaceae and Diaporthaceae associated with panicle and shoot blight of pistachio in California, USA. Fungal Diversity 67, 157179.

Chen SF, Pavlic D, Roux J, Slippers B, Xie YJ, et al. 2011 - Characterization of Botryosphaeriaceae from plantation-grown Eucalyptus species in South China. Plant Pathology 60, 739-751.

Conforto C, Lima NB, Garcete-Gomez JM, Camara MPS, Michereff S. 2016 - First report of cladode brown spot in cactus prickly pear caused by Neofusicoccum batangarum in Brazil. Plant Disease 100, 1238-1239.

Correia KC, Camara MPS, Barbosa MAG, Sales R, Agusti-Brisach C, et al. 2013 - Fungal trunk pathogens associated with table grape decline in Northeastern Brazil. Phytopathologia Mediterranea 52, 380-387.

Correia KC, Silva MA, de Morais MA Jr, Armengol J, Phillips AJL, et al. 2016a - Phylogeny, distribution and pathogenicity of Lasiodiplodia species associated with dieback of table grape in the main Brazilian exporting region. Plant pathology 65, 92-103.

Correia KC, Silva MA, Netto MSB, Vieira WAS, Câmara MPS, et al. 2016b - First report of grapevine dieback caused by Neoscytalidium hyalinum in Brazil. Plant Disease 100, 213213.

Cosoveanu A, Hernandez M, Iacomi-Vasilescu B, Zhang X, Shu S, et al. 2016 - Fungi as endophytes in Chinese Artemisia spp: juxtaposed elements of phylogeny, diversity and bioactivity. Mycosphere 7, 102-117.

Coutinho IBL, Freire FCO, Lima CS, Lima JS, Goncalvesc FJT, et al. 2016 - Diversity of genus Lasiodiplodia associated with perennial tropical fruit plants in northeastern Brazil. Plant Pathology Doi: 10.1111/ppa.12565. 
Crous PW, Groenewald JZ, Shivas RG, Edwards J, Seifert KA, et al. 2011 - Fungal Planet Description Sheets: 69-91. Persoonia 26, 108-156.

Crous PW, Müller MM, Sánchez RM, Giordano L, Bianchinotti MV, et al. 2015a - Resolving Tiarosporella spp. allied to Botryosphaeriaceae and Phacidiaceae. Phytotaxa 202, 073093.

Crous PW, Palm ME. 1999 - Reassessment of the anamorph genera Botryodiplodia, Dothiorella and Fusicoccum. Sydowia 51, 167-175.

Crous PW, Schumacher RK, Wingfield MJ, Lombard L, Giraldo A, et al. 2015b - Fungal Systematics and Evolution: FUSE 1. Sydowia 67, 81-118.

Crous PW, Slippers B, Wingfield MJ, Rheeder J, Marasas WFO, et al. 2006 - Phylogenetic lineages in the Botryosphaeriaceae. Studies in Mycology 55, 235-253.

Crous PW, Wingfield MJ, Guarro J, Cheewangkoon R, van der Bank M, et al. 2013 - Fungal Planet description sheets: 154-213. Persoonia 31, 188-296.

Crous PW, Wingfield MJ, Richardson DM, Le Roux JJ, Strasberg D, et al. 2016 - Fungal Planet description sheets: 400-468. Persoonia 36, 316-458.

Crous PW, Wingfield MJ, Schumacher RK, Summerell BA, Giraldo A, et al. 2014 - Fungal Planet description sheets: 281-319. Persoonia 33, 212-292.

Cruywagen EM, Slippers B, Roux J, Wingfield MJ. 2016 - Phylogenetic species recognition and hybridization in Lasiodiplodia: A case study on species from baobabs. Fungal Biology http://dx.doi.org/10.1016/j.funbio.2016.07.014.

Damm U, Crous PW, Fourie PH. 2007 - Botryosphaeriaceae as potential pathogens of Prunus species in South Africa, with descriptions of Diplodia africana and Lasiodiplodia plurivora sp. nov. Mycologia 99, 664-680.

Daranagama DA, Thambugala KM, Campino B, Alves A, Bulgakov TS, et al. 2016 Phaeobotryon negundinis sp. nov. (Botryosphaeriales) from Russia. Mycosphere 7, 933941.

De la Mora-Castaneda JG, Cibrian-Tovar D, Perez-Vera OA. 2014 - Neofusicoccum eucalyptorum (= Botryosphaeria eucalyptorum) and $N$. parvum: pathogens in eucalyptus plantations in Mexico. Revista Chapingo Serie Ciencias Forestales Y Del Ambiente 20, 187-197.

Denman S, Crous PW, Groenewald JZ, Slippers B, Wingfield BD, et al. 2003 - Circumscription of Botryosphaeria species associated with Proteaceae based on morphology and DNA sequence data. Mycologia 95, 294-307.

Denman S, Crous PW, Taylor JE, Kang J-C, Pascoe I, et al. 2000 - An overview of the taxonomic history of Botryosphaeria, and a re-evaluation of its anamorphs based on morphology and ITS rDNA phylogeny. Studies in Mycology 45, 129-140.

Diaz GA, Auger J, Besoain X, Bordeu E, Latorre BA. 2013 - Prevalence and pathogenicity of fungi associated with grapevine trunk diseases in Chilean vineyards. Ciencia e Investigation Agraria 40, 327-339.

Didier Begoude BA, Slippers B, Wingfield MJ, Roux J. 2010 - Botryosphaeriaceae associated with Terminalia catappa in Cameroon, South Africa and Madagascar. Mycological Progress 9, 101-123.

Dissanayake AJ, Camporesi E, Hyde KD, Phillips AJL, Fu CY, et al. 2016 - Dothiorella species associated with woody hosts in Italy. Mycosphere 7, 51-63.

Dissanayake AJ, Zhang W, Li XH, Zhou Y, Hyde KD, et al. 2015b - First report of Neofusicoccum mangiferae associated with grapevine dieback in China. Phytopathologia Mediterranea 54, 414-419.

Dissanayake AJ, Zhang W, Liu M, Chukeatirote E, Yan JY, et al. 2015a - Lasiodiplodia pseudotheobromae causes pedicel and peduncle discolouration of grapes in China. Australasian Plant Disease 10, 21.

Doilom M, Shuttleworth L, Roux J, Chukeatirote E, Hyde KD. 2014 - Barriopsis tectonae sp. nov. a new species of Botryosphaeriaceae from Tectona grandis (teak) in Thailand. Phytotaxa $176,081-091$. 
Doilom M, Shuttleworth LA, Roux J, Chukeatirote E, Hyde KD. 2015 - Botryosphaeriaceae associated with Tectona grandis (teak) in Northern Thailand. Phytotaxa 233, 1-26.

Doll DA, Rolshausen PE, Pouzoulet J, Michailides TJ. 2015 - First report of Dothiorella iberica causing trunk and scaffold cankers of almond in California. Plant Disease 99, 1185-1185.

Dreaden TJ, Black AW, Mullerin S, Smith JA. 2014 - First report of Diplodia quercivora causing shoot dieback and branch cankers on live oak (Quercus virginiana) in the United States. Plant Disease 98, 282.

Espinoza JG, Briceño EX, Chávez ER, Úrbez-Torres JR, Latorre BA. 2009 - Neofusicoccum sp. associated with stem canker \& dieback of blueberry in Chile. Plant Disease 93, 1187-1194.

Fan XL, Hyde KD, Liu JK, Liang YM, Tian CM. 2015 - Multigene phylogeny and morphology reveal Phaeobotryon rhois sp nov (Botryosphaeriales, Ascomycota). Phytotaxa 205, 90-98.

Farr DF, Elliot M, Rossman AY, Edmonds RL. 2005 - Fusicoccum arbuti sp. nov. causing cankers on Pacific madrone in western North America with notes on Fusicoccum dimidiatum, the correct name for Scytalidium dimidiatum and Nattrassia mangiferae. Mycologia 97, 730741.

Farr DF, Rossman AY. 2016. Fungal databases, systematic mycology and microbiology laboratory, ARS, USDA. (SMML database). https://nt.ars-grin.gov/fungaldatabases/. Accessed 21 September 2016.

Ferreira MC, Almeida V, Mariana de L, Zani CL, Junior PAS, et al. 2015 - Molecular phylogeny, diversity, symbiosis and discover of bioactive compounds of endophytic fungi associated with the medicinal Amazonian plant Carapa guianensis (Meliaceae). Biochemical Systematics and Ecology 59, 36-44.

Funk A. 1964 - Botryosphaeria tsugae sp. nov, causing dieback of western hemlock in British Columbia. Canadian Journal of Botany 42, 769-775.

Gardner DE. 1997 - Botryosphaeria mamane sp. nov. associated with witches'- brooms on the endemic forest tree Sophora chrysophylla in Hawaii. Mycologia 89, 298-303.

Giambra S, Piazza G, Alves A, Mondello V, Berbegal M, et al. 2016-Botryosphaeriaceae species associated with diseased loquat trees in Italy and description of Diplodia rosacearum sp. nov. Mycosphere 7, 978-989.

Gramaje D, Agustí-Brisach C, Pérez-Sierra A, Moralejo E, Olmo D, et al. 2012 - Fungal trunk pathogens associated with wood decay of almond trees on Mallorca (Spain). Persoonia 28, $1-13$.

Gure A, Slippers B, Stenlid J. 2005 - Seed-borne Botryosphaeria spp. from native Prunus and Podocarpus trees in Ethiopia, with a description of the anamorph Diplodia rosulata sp. nov. Mycological Research 109, 1005-1014.

Hall TA. 1999 - BioEdit: a user-friendly biological sequence alignment editor and analysis program for Windows 95/98/NT. Nucleic Acids Symposium Series 41, 95-98.

Huang SK, Tangthirasunun N, Phillips AJL, Dai DQ, Wanasinghe DN, et al. 2016 - Morphology and phylogeny of Neoscytalidium orchidacearum sp. nov. (Botryosphaeriaceae). Mycobiology 44, 79-84.

Hyde KD, Hongsanan S, Jeewon R, Bhat DJ, McKenzie EHC, et al. 2016 - Fungal diversity notes 367-491: taxonomic and phylogenetic contributions to fungal taxa. Fungal Diversity 80, 1270.

Inderbitzin P, Bostock RM, Trouillas FP, Michailides TJ. 2010 - A six locus phylogeny reveals high species diversity in Botryosphaeriaceae from California almond. Mycologia 102, 1350-1368.

Ismail AM, Cirvilleri G, Lombard L, Crous PW, Groenewald JZ, et al. 2013 - Characterisation of Neofusicoccum species causing mango dieback in Italy. Journal of Plant Pathology 95, 549557.

Ismail AM, Cirvilleri G, Polizzi G, Crous PW, Groenewald JZ, et al. 2012 - Lasiodiplodia species associated with dieback disease of mango (Mangifera indica) in Egypt. Australasian Plant Pathology 41, 649-660. 
Jami F, Slippers B, Wingfield MJ, Gryzenhout M. 2013 - Greater Botryosphaeriaceae diversity in healthy than associated diseased Acacia karroo tree tissues. Australasian Plant Pathology 42, 421-430.

Jami F, Slippers B, Wingfield MJ, Gryzenhout M. 2014 - Botryosphaeriaceae species overlap on four unrelated, native South African hosts. Fungal Biology 118, 168-179.

Jami F, Slippers B, Wingfield MJ, Loots MT, Gryzenhout M. 2015 - Temporal and spatial variation of Botryosphaeriaceae associated with Acacia karroo in South Africa. Fungal Ecology 15, 51-62.

Jami F, Slippers B, Wingfield MJ, Gryzenhout M. 2012 - Five new species of the Botryosphaeriaceae from Acacia karoo in South Africa. Cryptogamie Mycologie 33, 245266.

Jayawardena RS, Li XH, Xu W, Yan JY, Li HL, et al. 2016. First Report of Botryosphaeria dothidea causing leaf necrosis of Camellia sinensis in Fujian Province, China. Plant Disease 100, 854-854.

Jurick WM, Vico I, Gaskins VL, Janisiewicz WJ, Peter KA. 2013 - First report of Neofusicoccum ribis causing postharvest decay of apple fruit from cold storage in Pennsylvania. Plant Disease 97, 999-999.

Katoh K, Toh H. 2008 - Recent developments in the MAFFT multiple sequence alignment program. Briefings in Bioinformatics 9, 276-285.

Kong CS, Qiu XL, Yi KS, Yu XF, Yu L. 2010 - First report of Neofusicoccum vitifusiforme causing blueberry blight of blueberry in China. Plant Disease 94, 1373.

Konta S, Hongsanan S, Phillips AJL, Jones EBG, Boonmee S, et al. 2016b - Botryosphaeriaceae from palms in Thailand II - two new species of Neodeightonia, N. rattanica and $N$. rattanicola from Calamus (rattan palm). Mycosphere 7, 950-961.

Konta S, Phillips AJL, Bahkali AH, Jones EBG, Eungwanichayapant DP, et al. 2016a Botryosphaeriaceae from palms in Thailand - Barriopsis archontophoenicis sp. nov, from Archontophoenix alexandrae. Mycosphere 7, 921-932.

Krishnapillai N, Wijeratnam RSW. 2015 - First report of Neofusicoccum mediterraneum causing stem end rot on Karuthakolumban mangoes. Plant Disease 99, 1858-1858.

Lawrence DP, Hand FP, Gubler WD, Trouillas FP. 2016 - Botryosphaeriaceae species associated with dieback and canker disease of bay laurel in northern California with the description of Dothiorella californica sp. nov. Fungal Biology doi: 10.1016/j.funbio.2016.09.005.

Lazzizera C, Frisullo S, Alves A, Phillips AJL. 2008 - Morphology, phylogeny and pathogenicity of Botryosphaeria and Neofusicoccum species associated with drupe rot of olives in southern Italy. Plant Pathology 57, 948-956.

Li GJ, Hyde KD, Zhao RL, Hongsanan S, Abdel-Aziz FA, et al. 2016a - Fungal diversity notes 253-366: taxonomic and phylogenetic contributions to fungal taxa. Fungal Diversity 78: 1237.

Li GQ, Arnold RJ, Liu FF, Li JQ, Chen SF. 2015 - Identification and Pathogenicity of Lasiodiplodia Species from Eucalyptus urophyllaxgrandis, Polyscias balfouriana and Bougainvillea spectabilis in Southern China. Journal of Phytopathology 163, 956-967.

Li GQ, Liu FF, Li JQ, Liu QL, Chen SF. 2016b - Characterization of Botryosphaeria dothidea and Lasiodiplodia pseudotheobromae from English Walnut in China. Journal of Phytopathology $164,348-353$.

Li W, Liu J, Bhat DJ, Camporesi E, Xu J, et al. 2014 - Introducing the novel species, Dothiorella symphoricarposicola, from snowberry in Italy. Cryptogamie Mycology 35, 257-270.

Ligoxigakis EK, Markakis EA, Papaioannou IA, Typas MA. 2013 - First report of palm rot of Phoenix spp caused by Neodeightonia phoenicum in Greece. Plant Disease 97, 286-286.

Linaldeddu BT, Alves A, Phillips AJL. 2016b - Sardiniella urbana gen. et sp. nov., a new member of the Botryosphaeriaceae isolated from declining Celtis australis trees in Sardinian streetscapes. Mycosphere 7, 893-905. 
Linaldeddu BT, Deidda A, Scanu B, Franceschini A, Alves A, et al. 2016a - Phylogeny, morphology and pathogenicity of Botryosphaeriaceae, Diatrypaceae and Gnomoniaceae associated with branch diseases of hazelnut in Sardinia (Italy). European Journal of Plant Pathology Doi: 10.1007/s10658-016-0912-z.

Linaldeddu BT, Deidda A, Scanu B, Franceschini A, Serra S, et al. 2015 - Diversity of Botryosphaeriaceae species associated with grapevine and other woody hosts in Italy, Algeria and Tunisia, with descriptions of Lasiodiplodia exigua and Lasiodiplodia mediterranea sp. nov. Fungal Diversity 71, 201-214.

Linaldeddu BT, Franceschini A, Alves A, Phillips AJL. 2013 - Diplodia quercivora sp. nov.: a new species of Diplodia found on declining Quercus canariensis trees in Tunisia. Mycologia 105, 1266-1274.

Linaldeddu BT, Maddau L, Franceschini A, Alves A, Phillips AJL. 2016c - Botryosphaeriaceae species associated with lentisk dieback in Italy and description of Diplodia insularis sp. nov. Mycosphere 7, 962-977.

Linaldeddu BT, Scanu B, Maddau L, Franceschini A. 2014 - Diplodia corticola and Phytophthora cinnamomi: the main pathogens involved in holm oak decline on Caprera island (Italy). Forest Pathology 44, 191-200.

Liu JK, Phookamsak R, Doilom M, Wikee S, Li YM, et al. 2012 - Towards a natural classification of Botryosphaeriales. Fungal Diversity 57, 149-210.

Lopes A, Phillips AJL, Alves A (2016) - Mating type genes in the genus Neofusicoccum: mating strategies and usefulness in species delimitation. Fungal Biology Doi 10.1016/j.funbio.2016.08.011

Lopes UP, Zambolim L, Pinho DB, Barros AV, Costa H, et al. 2014 - Postharvest rot and mummification of strawberry fruits caused by Neofusicoccum parvum and $N$. kwambonambiense in Brazil. Tropical Plant Pathology 39, 178-183.

Luque J, Martos S, Phillips AJL. 2005 - Botryosphaeria viticola sp. nov. on grapevines: a new species with a Dothiorella anamorph. Mycologia 97, 1111-1121.

Lynch SC, Eskalen A, Zambino PJ, Mayorquin JS, Wang DH. 2013 - Identification and pathogenicity of Botryosphaeriaceae species associated with coast live oak (Quercus agrifolia) decline in southern California. Mycologia 105, 125-140.

Lynch SC, Zambino PJ, Scott TA, Eskalen A. 2014 - Occurrence, incidence and associations among fungal pathogens and Agrilus auroguttatus, and their roles in Quercus agrifolia decline in California. Forest Pathology 44, 62-74.

Machado AR, Pinho DB, de Oliveira SAS, Pereira OL. 2014b - New occurrences of Botryosphaeriaceae causing black root rot of cassava in Brazil. Tropical Plant Pathology 39, 464-470.

Machado AR, Pinho DB, Pereira OL. 2014a - Phylogeny, identification and pathogenicity of the Botryosphaeriaceae associated with collar and root rot of the biofuel plant Jatropha curcas in Brazil, with a description of new species of Lasiodiplodia. Fungal Diversity 67, 231-247.

Marincowitz S, Groenewald JZ, Wingfield MJ, Crous PW. 2008 - Species of Botryosphaeriaceae occurring on Proteaceae. Persoonia 21, 111-118.

Marques MW, Lima NB, de Morais MA Jr, Barbosa MAG, Souza BO, et al. 2013a - Species of Lasiodiplodia associated with mango in Brazil. Fungal Diversity 61, 181-193.

Marques MW, Lima NB, de Morais MA Jr, Michereff SJ, Phillips AJL, et al. 2013b. Botryosphaeria, Neofusicoccum, Neoscytalidium and Pseudofusicoccum species associated with mango in Brazil. Fungal Diversity 61, 195-208.

Marques MW, Lima NB, Michereff SJ, Câmara MPS, Souza CRB. 2012 - First report of mango dieback caused by Pseudofusicoccum stromaticum in Brazil. Plant Disease 96, 144.

Mcgregor RR, Sakalidis ML, Hamelin RC. 2016 - Neofusicoccum arbuti: a hidden threat to Arbutus menziesii characterized by widespread latent infections and a broad host range. Canadian Journal of Plant Pathology 38, 70-81. 
Mehl JWM, Slippers B, Roux J, Wingfield MJ. 2011 - Botryosphaeriaceae associated with Pterocarpus angolensis (kiaat) in South Africa. Mycologia 103, 534-553.

Mehl JWM, Slippers B, Roux J, Wingfield MJ. 2014 - Botryosphaeriaceae associated with dieback of Schizolobium parahyba trees in South Africa and Ecuador. Forest Pathology 44, 396-408.

Michailides TJ, Morgan DP. 2016 - Association of Botryosphaeria panicle and shoot blight of Pistachio with injuries of fruit caused by Hemiptera insects and birds. Plant Disease 100, 1405-1413.

Mishra A, Gond SK, Kumar A, Sharma VK, Verma SK, et al. 2012 - Season and tissue type affect fungal endophyte communities of the Indian medicinal plant Tinospora cordifolia more strongly than geographic location. Microbial Ecology 64, 388-398.

Mohali SR, Slippers B, Wingfield MJ. 2006 - Identification of Botryosphaeriaceae from Eucalyptus, Acacia and Pinus in Venezuela. Fungal Diversity 25, 103-125.

Mohammadi H, Kazemi S, Farahmand H. 2014 - Phaeoacremonium and Botryosphaeriaceae species associated with cypress (Cupressus sempervirens L.) decline in Kerman province (Iran). Phytopathologia Mediterranea 53, 27-39.

Mohammadi H. 2014 - Phaeoacremonium spp and Botryosphaeriaceae spp associated with date palm (Phoenix dactylifera L) decline in Iran. Journal of Phytopathology 162, 575-581.

Mondello V, Lo PS, Conigliaro G, Alfonzo A, Torta L, et al. 2013 - First report of Neofusiccoccum vitifusiforme and presence of other Botryosphaeriaceae species associated with Botryosphaeria dieback of grapevine in Sicily (Italy). Phytopathologia Mediterranea 52, 388-396.

Netto MSB, Assuncao IP, Lima GSA, Marques MW, Lima WG, et al. 2014 - Species of Lasiodiplodia associated with papaya stem-end rot in Brazil. Fungal Diversity 67, 127-141.

Netto MSB, Lima WG, Correia KC, Da Silva CFB, Thon M, et al. 2016 - Analysis of phylogeny, distribution, and pathogenicity of Botryosphaeriaceae species associated with gummosis of Anacardium in Brazil, with a new species of Lasiodiplodia. Fungal Biology http://dx.doi.org/10.1016/j.funbio.2016.07.006.

Ngobisa AICN, Abidin MAZ, Wong MY, Wan NM. 2013 - Neofusicoccum ribis associated with leaf blight on rubber (Hevea brasiliensis) in Peninsular Malaysia. Plant Pathology Journal 29, 10-16.

Ni HF, Yang HR, Chen RS, Rueyfen L, Tinghsuan H. 2012 - New Botryosphaeriaceae fruit rot of mango in Taiwan: identification and pathogenicity. Botanical Studies 53, 467-478.

Niekerk JM van, Bester W, Halleen F, Crous PW, Fourie PH. 2010 - First report of Lasiodiplodia crassispora as a pathogen of grapevine trunks in South Africa. Plant Disease 94, 1063.

Niekerk JM van, Crous PW, Groenewald JZ, Fourie PH, Halleen F. 2004 - DNA phylogeny, morphology and pathogenicity of Botryosphaeria species on grapevines. Mycologia 96, 781-798.

Nogueira Junior AF, Fischer IH, Braganca CAD, Massola Jr NS, Amorim L. 2016 - Identification of Botryosphaeriaceae species that cause stylar-end rot of guavas and characterisation of the disease monocycle. European Journal of plant pathology 144, 271-287.

Nylander JAA. 2004 - MrModeltest 2.0. Program distributed by the author. Evolutionary Biology Centre, Uppsala University.

Osorio JA, Crous CJ, Beer ZWD, Wingfield MJ, Roux J. 2016 - Endophytic Botryosphaeriaceae, including five new species, associated with mangrove trees in South Africa. Fungal Biology doi:10.1016/j.funbio.2016.09.004J.

Palou L, Montesinos-Herrero C, Besada C, Taberner V. 2013 - Postharvest fruit rot of Persimmon (Diospyros kaki) in Spain caused by Lasiodiplodia theobromae and Neofusicoccum spp. Journal of Phytopathology 161, 625-631.

Pavlic D, Slippers B, Coutinho TA, Gryzenhout M, Wingfield MJ. 2004 - Lasiodiplodia gonubiensis sp. nov., a new Botryosphaeria anamorph from native Syzygium cordatum in South Africa. Studies in Mycology 50, 313-322. 
Pavlic D, Slippers B, Coutinho TA, Wingfield MJ. 2007 - Botryosphaeriaceae occurring on native Syzygium cordatum in South Africa and their potential threat to Eucalyptus. Plant Pathology 56, 624-636.

Pavlic D, Slippers B, Coutinho TA, Wingfield MJ. 2009 - Molecular and phenotypic characterization of three phylogenetic species discovered within the Neofusicoccum parvum/N. ribis complex. Mycologia 101, 636-647.

Pavlic D, Wingfield MJ, Barber P, Slippers B, Hardy GEStJ, et al. 2008 - Seven new species of the Botryosphaeriaceae from baobab and other native trees in Western Australia. Mycologia 100, 851-866.

Pavlic-Zupanc D, Pis `ur B, Slippers B, Wingfield M, Jurc D. 2015 - Molecular and morphological characterization of Dothiorella species associated with dieback of Ostrya carpinifolia in Slovenia and Italy. Phytopathologia Mediterranea 54, 222-231.

Pérez CA, Wingfield MJ, Slippers B, Altier NA, Blanchette RA. 2010 - Endophytic and cankerassociated Botryosphaeriaceae occurring on non-native Eucalyptus and native Myrtaceae trees in Uruguay. Fungal Diversity 41, 53-69.

Pérez SF, Merino-Gergichevich C, Guerrero JC. 2014. Detection of Neofusicoccum nonquaesitum causing dieback and canker in highbush blueberry from Southern Chile. Journal of Soil Science and Plant Nutrition 14, 581-588.

Phillips AJL, Alves A, Abdollahzadeh J, Slippers B, Wingfield MJ, et al. 2013 - The Botryosphaeriaceae: genera and species known from culture. Studies in Mycology 76, 51167.

Phillips AJL, Alves A, Pennycook SR, Johnston PR, Ramaley A, et al. 2008 - Resolving the phylogenetic and taxonomic status of dark-spored teleomorph genera in the Botryosphaeriaceae. Persoonia 21, 29-55.

Phillips AJL, Lopes J, Abdollahzadeh J, Bobev S, Alves A. 2012 - Resolving the Diplodia complex on apple and other Rosaceae hosts. Persoonia 29, 29-38.

Phillips AJL, Oudemans PV, Correia A, Alves A. 2006 - Characterization and epitypification of Botryosphaeria corticis, the cause of blueberry cane canker. Fungal Diversity 21, 141-155.

Pillay K, Slippers B, Wingfield MJ, Gryzenhout M. 2013 - Diversity and distribution of coinfecting Botryosphaeriaceae from Eucalyptus grandis and Syzygium cordatum in South Africa. South African Journal of Botany 84, 38-43.

Pitt WM, Huang R, Steel CC, Savocchia S. 2013a - Pathogenicity and epidemiology of Botryosphaeriaceae species isolated from grapevines in Australia. Australasian Plant Pathology 42, 573-582.

Pitt WM, Úrbez-Torres JR, Trouillas FP. 2013b - Dothiorella vidmadera, a novel species from grapevines in Australia and notes on Spencermartinsia. Fungal Diversity 61, 209-219.

Pitt WM, Úrbez-Torres JR, Trouillas FP. 2015 - Dothiorella and Spencermartinsia, new species and records from grapevines in Australia. Australasian Plant Pathol 44, 43-56.

Poczai P, Varga I, Hyvonen J. 2015 - Internal transcribed spacer (ITS) evolution in populations of the hyperparasitic European mistletoe pathogen fungus, Sphaeropsis visci (Botryosphaeriaceae): The utility of ITS2 secondary structures. Gene 558, 54-64.

Poletto T, Gonzatto Maciel C, Muniz M, Blume E, et al. 2016 - First report of stem canker caused by Lasiodiplodia subglobosa on Carya illinoinensis in Brazil. Plant Disease 100, 10161017.

Punithalingam E. 1969 - Studies on Sphaeropsidales in culture. Mycological Papers 119, 1-24.

Qiu Y, Steel CC, Ash GJ, Savocchia S. 2011 - Survey of Botryosphaeriaceae associated with grapevine decline in the Hunter Valley and Mudgee grape growing regions of New South Wales. Australasian Plant Pathology 40, 1-11.

Rabari V, Rakhashiya P, Patel P, Thaker V. 2016 - First Report of Botryosphaeria dothidea on Mangifera indica L in Gujarat. Journal of Phytopathology 164, 286-289. 
Ray JD, Lanoiselet V, Burgess TI. 2010 - First record of Neoscytalidium dimidiatum and Neoscytalidium novaehollandiae on Mangifera indica and Ficus carica in Australia. Australasian Plant Disease Notes 5, 48-50.

Rodrígez-Galvéz E, Guerrero P, Barradas C, Crous PW, Alves A. 2016 - Phylogeny and pathogenicity of Lasiodiplodia species associated with dieback of mango in Peru. Fungal Biology http://dx.doi.org/10.1016/j.funbio.2016.06.004.

Rojas EI, Herre EA, Mejía LC, Arnold AE, Chaverri P, et al. 2008 - Endomelanconiopsis, a new anamorph genus in the Botryosphaeriaeae. Mycologia 100, 760-775.

Ronquist F, Huelsenbeck JP. 2003 - MrBayes 3: Bayesian phylogenetic inference under mixed models. Bioinformatics 19, 1572-1574.

Rooney-Latham S, Soriano MC. 2016 - First Report of Neofusicoccum nonquaesitum Causing Branch Dieback of Apple in California. Plant Disease 100, 1012-1012.

Rooney-Latham S, Tidwell TE, Blomquist CL, Peek KS. 2012 -. First report of Neofusicoccum nonquaesitum causing branch cankers on giant sequoia (Sequoiadendron giganteum) in North America. Plant Disease 96, 905.

Rosado AWC, Machado AR, Freire FCO, Pereira OL. 2016 - Phylogeny, identification, and pathogenicity of Lasiodiplodia associated with postharvest stem-end rot of coconut in Brazil. Plant Disease 100, 561-568.

Sakalidis ML, Hardy GEStJ, Burgess TI. 2011 - Use of the genealogical sorting index (GSI) to delineate species boundaries in the Neofusicoccum parvum- Neofusicoccum ribis species complex. Molecular Phylogenetics and Evolution 60, 333-344.

Sandoval-Sanchez M, Nieto-Angel D, Sergio SIJ, Téliz-Ortiz D, Orozco-Santos M, et al. 2013 Fungi associated to stem-end rot and dieback of mango (Mangifera indica L.). Agrocienica 47, 61-73.

Serrato-Diaz LM, Rivera-Vargas L, French-Monar RD. 2014 - First Report of Neofusicoccum mangiferae Causing Rachis Necrosis and Inflorescence Blight of Mango (Mangifera indica) in Puerto Rico. Plant Disease 98, 570-571.

Sharma R, Kulkarni G, Shouche YS. 2013 - Pseudofusicoccum adansoniae isolated as an endophyte from Jatropha podagrica: new record for India. Mycotaxon 123, 39-45.

Shear CL, Stevens NE, Wilcox MS. 1923 - Botryosphaeria and Physalospora in the Eastern United States. Mycologia 17, 98-107.

Shetty KG, Minnis AM, Rossman AY, Jayachandran K. 2011 - The Brazilian peppertree seedborne pathogen, Neofusicoccum batangarum, a potential biocontrol agent. Biological Control 56, 91-97.

Shivas RG, Tree DJ, Tan YP, Ballard EL. 2009 - Dothiorella thripsita. Fungal Planet 32, Persoonia 22, 168-169.

Silvestro D, Michalak I. 2010 - RaxmlGUI: a graphical front-end for RAxML. Available from: http://sourceforge.net/projects/raxmlgui/ (accessed 25 September 2016)

Slippers B, Boissin E, Phillips AJL, Groenewald JZ, Wingfield MJ, et al. 2013 - Phylogenetic lineages in the Botryosphaeriales: A systematic and evolutionary framework. Studies in Mycology 76, 31-49.

Slippers B, Crous PW, Denman S, Coutinho TA, Wingfield BD, et al. 2004a - Combined multiple gene genealogies and phenotypic characters differentiate several species previously identified as Botryosphaeria dothidea. Mycologia 96, 83-101.

Slippers B, Fourie G, Crous PW, Coutinho TA, Wingfield BD, et al. 2004b - Speciation and distribution of Botryosphaeria sp. on native and introduced Eucalyptus trees in Australia and South Africa. Studies in Mycology 50, 343-358.

Slippers B, Johnson GI, Crous PW, Coutinho TA, Wingfield BD, et al. 2005 - Phylogenetic and morphological re-evaluation of the Botryosphaeria species causing diseases of Mangifera indica. Mycologia 97, 99-110.

Slippers B, Roux J, Wingfield MJ, Van der Walt FJJ, Jami F, et al. 2014 - Confronting the constraints of morphological taxonomy in the Botryosphaeriales. Persoonia 33, 155-168. 
Smith DR, Stanosz GR. 2001 - Molecular and morphological differentiation of Botryosphaeria dothidea (anamorph Fusicoccum aesculi) from some other fungi with Fusicoccum anamorphs. Mycologia 93, 505-515.

Solel Z, Madar Z, Kimchi M, Golan Y. 1987 - Diplodia canker of cypress. Canadian Journal of Plant Pathology 9, 115-118.

Stevens NE. 1926 - Two species of Physalospora on citrus and other hosts. Mycologia 18, 206217.

Summerell BA, Groenewald JZ, Carnegie AJ, Summerbell RC, Crous PW. 2006 - Eucalyptus microfungi known from culture Alysidiella, Fusculina and Phlogicylindrium genera nova, with notes on some other poorly known taxa. Fungal Diversity 23, 323-350.

Sutton BC, Marasas WFO. 1976 - Observations on Neottiosporina and Tiarosporella. Transactions of the British Mycological Society 67, 69-76.

Sutton BC. 1980 - The Coelomycetes: Fungi imperfecti with pycnidia, acervuli and stromata. Commonwealth Mycological Institute, Kew, Surrey, England.

Swofford DL. 2002 - PAUP* 4.0: phylogenetic analysis using parsimony (*and other methods). Sinauer Associates, Sunderland.

Taylor K, Barber PA, Hardy GEStJ, Burgess TI. 2009 - Botryosphaeriaceae from tuart (Eucalyptus gomphocephala) woodland, including descriptions of four new species. Mycological Research 113, 337-353.

Tennakoon DS, Phillips AJL, Phookamsak R, Ariyawansa HA, Bahkali AH, et al. 2016 - Sexual morph of Lasiodiplodia pseudotheobromae (Botryosphaeriaceae, Botryosphaeriales, Dothideomycetes) from China. Mycosphere 7, 990-1000.

Thambugala KM, Daranagama DA, Camporesi E, Singtripop C, Liu ZY, et al. 2014 - Multi-locus phylogeny reveals the sexual state of Tiarosporella in Botryosphaeriaceae. Cryptogam Mycol 35, 359-367.

Theissen F, Sydow H. 1918 - Vorentwürfe zu den Pseudosphaeriales. Annales Mycologici 16, 134.

Thompson JD, Gibson TJ, Plewniak F, Jeanmougin F, Higgins DG. 1997 - The Clustal X windows interface: flexible strategies for multiple sequence alignment aided by quality analysis tools. Nucleic Acids Research 24, 4876-4882.

Thynne E, McDonald MC, Evans M, Wallwork H, Neate S, et al 2015 - Re-classification of the causal agent of white grain disorder on wheat as three separate species of Eutiarosporella. Australasian Plant Pathology 44, 527-539.

Trakunyingcharoen T, Cheewangkoon R, To-anun C. 2015a - Phylogenetic study of the Botryosphaeriaceae species associated with avocado and para rubber in Thailand. Chiang Mai Journal of Science 42, 104-116.

Trakunyingcharoen T, Lombard L, Groenewald JZ, Cheewangkoon R, To-Anun C, et al. 2015b Caulicolous Botryosphaeriales from Thailand. Persoonia 34, 87-99.

Triki MA, HadjTaieb SK, Cheffi M, Gharbi Y, Rhouma A. 2015 - First report of dieback of Olive trees caused by Neofusicoccum australe in Tunisia. Journal of Plant Pathology 97, 212-212.

Turkolmez S, Dervis S, Ciftci O, Serçe CU. 2016 - First Report of Canker and Dieback Caused by Botryosphaeria dothidea on Apple in Turkey. Plant Disease 100, 1237-1238.

Úrbez-Torres JR, Boule J, O'Gorman DT. 2016 - First Report of Diplodia seriata and D. mutila Causing Apple Dieback in British Columbia. Plant Disease 100, 1243-1244.

Úrbez-Torres JR, Gubler WD, Luque J. 2007 - First report of Botryosphaeria iberica and B. viticola associated with grapevine decline in California. Plant Disease 91, 772.

Úrbez-Torres JR, Peduto F, Rooney-Latham S, Gubler WD. 2010 - First report of Diplodia corticola causing grapevine (Vitis vinifera) cankers and trunk cankers and dieback of canyon live oak (Quercus chrysolepis) in California. Plant Disease 94, 785.

Úrbez-Torres JR, Peduto F, Striegler RK, Urrearomero KE, Rupe JC, et al. 2012 - Characterization of fungal pathogens associated with grapevine trunk diseases in Arkansas and Missouri. Fungal Diversity 52, 169-189. 
Úrbez-Torres JR, Peduto F, Vossen PM, Krueger WH, Gubler WD. 2013 - Olive twig and branch dieback: etiology, incidence, and distribution in California. Plant Disease 97, 231-244.

Valencia D, Torres C, Camps R, López E, Celisdiez JL, et al. 2015 - Dissemination of Botryosphaeriaceae conidia in vineyards in the semiarid Mediterranean climate of the Valparaiso Region of Chile. Phytopathologia Mediterranea 54, 394-402.

van der Linde JA, Six DL, Wingfield MJ, Roux J. 2011 - Lasiodiplodia species associated with dying Euphorbia ingens in South Africa. Southern Forests 73, 165-173.

Varga I, Poczai P, Cernak I, Hyvönen J. 2014 - Application of direct PCR in rapid rDNA ITS haplotype determination of the hyperparasitic fungus Sphaeropsis visci (Botryosphaeriaceae). Springerplus 3, 569.

Vasic M, Duduk N, Vico, Ivanovic' MS. 2013 - First Report of Botryosphaeria dothidea Causing white rot of apple fruit in Serbia. Plant Disease 97, 1659-1659.

Verkley GJM, Aa HA van der. 1997 - Endomelanconium microsporum, a new coelomycete isolated from soil in Papua New Guinea. Mycologia 89, 967-970.

Wang X, Li YX, Dong HX, Jia XZ, Zhang XY. 2015 - First Report of Botryosphaeria dothidea causing canker of Acer platanoides in China. Plant Disease 99, 1857-1857.

Wet J de, Burgess T, Slippers B, Preisig O, Wingfield BD, et al. 2003 - Multiple gene genealogies and microsatellite markers reflect relationships between morphotypes of Sphaeropsis sapinea and distinguish a new species of Diplodia. Mycological Research 107, 557-566.

Wet J de, Slippers B, Preisig O, Wingfield BD, Tsopelas P, et al. 2009 - Molecular and morphological characterization of Dothiorella casuarinae sp. nov. and other Botryosphaeriaceae with diplodia-like conidia. Mycologia 101, 503-511.

Wikee S, Lombard L, Nakashima C, Motohashi K, Chukeatirote E, et al. 2013 - A phylogenetic reevaluation of Phyllosticta (Botryosphaeriales). Studies in Mycology 76, 1-29.

Wright AF, Harmon PF. 2010 - Identification of species in the Botryosphaeriaceae family causing stem blight on southern highbush blueberry in Florida. Plant Disease 94, 966-971.

Xu C, Wang C, Ju L, Zhang R, Biggs AR, et al. 2015a - Multiple locus genealogies \& phenotypic characters reappraise causal agents of apple ring rot in China. Fung Dive 71, 215-231.

$\mathrm{Xu} \mathrm{C}$, Zhang $\mathrm{H}$, Zhou Z, Hu T, Wang S. 2015b - Identification and distribution of Botryosphaeriaceae species associated with blueberry stem blight in China. European Journal of Plant Pathology 143, 737-752.

Yan JY, Xie Y, Zhang W, Wang, Liu JK, et al. 2013 - Species of Botryosphaeriaceae involved in grapevine dieback in China. Fungal Diversity 61, 221-236.

Yan YC, Zhang ZX, Song YJ, Deng DF, Liu ZY. 2016 - First report of Prunus serrulata stem canker caused by Botryosphaeria dothidea in China. Plant Disease 100, 858-858.

Zhai L, Zhang M, Lv G, Chen X, Jia N, et al. 2014 - Biological and molecular characterization of four Botryosphaeria species isolated from pear plants showing stem wart and stem canker in China. Plant Disease 98, 716-726.

Zhang M, He W, Wu JR, Zhang Y. 2016 - Two new species of Spencermartinsia (Botryosphaeriaceae, Botryosphaeriales) from China. Mycosphere 7, 942-949.

Zhang R, Guo X, Sun G, Tang M, Gleason ML. 2009 - Dothiorella viticola on Populus cathayana in China: a new record. Mycotaxon 109, 129-135.

Zhou Y, Dou Z, He W, Zhang X, Zhang Y. 2016 - Botryosphaeria sinensia sp nov., a new species from China. Phytotaxa 245, 43-50.

Zhou Y, Gong G, Cui Y, Zhang DX, Chang XL, et al. 2015 - Identification of Botryosphaeriaceae species causing kiwifruit rot in Sichuan Province, China. Plant Disease 99, 699-708.

Zhu H, Qin WQ, Liu L, Yan W, Ca OCG. 2015 - First report of leaf spot of fishtail palm (Caryota mitis) caused by Lasiodiplodia jatrophicola in China. Plant Disease 99, 1038-1038.

Zlatkovic M, Keca N, Wingfield MJ, Jami F, Slippers B. 2016 - Botryosphaeriaceae associated with the die-back of ornamental trees in the Western Balkans. Antonie Van Leeuwenhoek International of General Molecular Microbiology 109, 543-564. 